\title{
Quality of life and health status in sarcoidosis
}

\author{
Citation for published version (APA):
}

Wirnsberger, R. (1999). Quality of life and health status in sarcoidosis. [Doctoral Thesis, Maastricht University]. https://doi.org/10.26481/dis.19990114rw

Document status and date:

Published: 01/01/1999

DOI:

10.26481/dis.19990114rw

Document Version:

Publisher's PDF, also known as Version of record

\section{Please check the document version of this publication:}

- A submitted manuscript is the version of the article upon submission and before peer-review. There can be important differences between the submitted version and the official published version of record.

People interested in the research are advised to contact the author for the final version of the publication, or visit the DOI to the publisher's website.

- The final author version and the galley proof are versions of the publication after peer review.

- The final published version features the final layout of the paper including the volume, issue and page numbers.

Link to publication

\footnotetext{
General rights rights.

- You may freely distribute the URL identifying the publication in the public portal. please follow below link for the End User Agreement:

www.umlib.nl/taverne-license

Take down policy

If you believe that this document breaches copyright please contact us at:

repository@maastrichtuniversity.nl

providing details and we will investigate your claim.
}

Copyright and moral rights for the publications made accessible in the public portal are retained by the authors and/or other copyright owners and it is a condition of accessing publications that users recognise and abide by the legal requirements associated with these

- Users may download and print one copy of any publication from the public portal for the purpose of private study or research.

- You may not further distribute the material or use it for any profit-making activity or commercial gain

If the publication is distributed under the terms of Article $25 \mathrm{fa}$ of the Dutch Copyright Act, indicated by the "Taverne" license above, 


\section{Quality of life and health status in sarcoidosis}


(C): Ruth Wirnsberger, Utrecht 1999

ISBN: 90-9012209-5

Lay-out: Mandare Office Management \& Congress Organization Printed by: PrintPartners Ipskamp, Enschede 


\title{
Quality of life and health status in sarcoidosis
}

\author{
PROEFSCHRIFT
}

ter verkrijging van de graad van doctor aan de Universiteit Maastricht, op gezag van de Rector Magnificus, Prof.dr. A.C. Nieuwenhuijzen Kruseman

volgens het besluit van het College van Decanen, in het openbaar te verdedigen op donderdag 14 januari 1999 om 16.00 uur

door

\section{Ruth Margarethe Wirnsberger}

geboren te Villach 


\section{Promotor:}

Prof.dr. E.F.M. Wouters

\section{Co-promotores:}

Dr. M. Drent

Dr. J. de Vries

\section{Beoordelingscommissie:}

Prof.dr. J.M.J.P. van der Linden (voorzitter)

Prof.dr. U. Costabel (Ruhrlandklinik, Essen, Duitsland)

Prof.dr. M. Demedts (Katholieke Universiteit Leuven, België)

Prof.dr. H. Huijer Abu-Saad

Prof.dr. C.P. van Schayck

Het verschijnen van dit proefschrift werd mede mogelijk gemaakt door financiële steun van:

Astra, Boehringer Ingelheim, GlaxoWellcome, MSD, Novartis. 


\section{CONTENTS}

Chapter 1 General introduction 1

$\begin{array}{ll}\text { Chapter } 2 \text { Aims of the study } & 25\end{array}$

Chapter 3 Clinical presentation of sarcoidosis in the

Netherlands. An epidemiological study

Chapter 4 Quality of life and depressive symptoms in patients suffering from sarcoidosis

Chapter 5 Evaluation of quality of life in sarcoidosis patients

Chapter 6 Impairment of quality of life: rheumatoid arthritis versus sarcoidosis

Chapter 7 Relationship between respiratory muscle function and quality of life in sarcoidosis

Chapter 8 Association of fatigue with an acute phase response in sarcoidosis

Chapter 9 General discussion

Chapter 10 Summary

Samenvatting (summary in Dutch)

Zusammenfassung (summary in German)

Abbreviations

Appendix Questionnaires

A.1 Beck Depression Inventory (BDI)

A.2 Positive Affect Negative Affect Schedule (PANAS)

A.3 Sickness Impact Profile (SIP)

A.4 World Health Organization of Quality of Life assessment instrument (WHOQOL100)

Nawoord/acknowledgements 

Jede Krankheit kann man

Seelenkrankheit nennen

Novalis, deutscher Dichter

(1772-1801)

Aan Sjoerd 
Chapter 1

General introduction 


\section{GENERAL INTRODUCTION}

\section{Features of sarcoidosis}

\section{Introduction}

Sarcoidosis is a multi-organ disorder of unknown origin [1] that is characterized by a specific morphological hallmark, the noncaseating granuloma [2]. Although the most commonly involved organs are the lungs and bilateral hilar lymph nodes $[2,3]$, practically any organ of the body may be affected by sarcoidosis [4]. The eyes, skin, liver, spleen, salivary glands, heart, and muscles are frequently affected. Involvement of the bones, nose, throat, and the nervous system is less common [5,6]. Sarcoidosis has been known for more than 100 years; it was first described by the dermatologist Hutchinson, and several years later by two other dermatologists, Besnier and Boeck [7]. The disorder is also known as Morbus Besnier/Boeck.

\section{Prevalence}

Although sarcoidosis occurs worldwide, the prevalence and the course of the disease vary across countries and ethnic groups [4]. In Denmark, Sweden, Ireland, and among A frican Americans the prevalence is more than 50/100,000, compared with less than 10/100,000, in Spain, Italy, Argentina, and Australia [8]. In the Netherlands the prevalence is estimated to be $20-30 / 100,000$ [8]. The prevalence of sarcoidosis may be underestimated in countries without chest radiograph screening programs because the disease is often asymptomatic $[2,9]$. Sarcoidosis commonly affects young and middle-aged adults [5]. The peak incidence occurs between 20 and 40 years of age in both sexes, with a second lower and broader peak in women between 45 and 65 years of age $[2,10]$. The disease appears to be more common in women than in men $[9,10$ 12]. Genetic factors have been found to play a role; family clustering occurs 
more frequently in families of African descent than in Caucasian families $[2,13]$.

\section{Pathogenesis and immunology}

Noncaseating granulomas are the pathological characteristic of sarcoidosis. The initial lesion in the lung, however, appears to be a mononuclear cell alveolitis with an accumulation of alveolar macrophages and activated $T$ lymphocytes $[4,8]$. Although no antigen has been identified, the pathological features suggest an antigen-driven process. Recently, more evidence has been obtained concerning the immunological events that lead to the granuloma formation and the evolution towards fibrosis in sarcoidosis patients [14]. It is well known that several mediators (cytokines), produced by activated $T$ lymphocytes, are responsible for this immunoinflammatory process. Mononuclear inflammatory cells accumulate in the target organ and redistribution and local proliferation accounts for the increase in the number of these immunocompetent cells [2]. Sarcoid granulomas, which vary in activity and differentiation, are composed of monocytes-macrophages, which may differentiate into epitheloid cells and multinucleated giant cells [2,14]. A mixture of lymphocytes and plasma cells form a rim around the accumulation of these cells $[2,4]$ (fig. I).

The granulomas are usually microscopic, but, they may form masses $3-4 \mathrm{~cm}$ in diameter [4]. Granulomas may remain for months to years and, finally, resolve. However, if repair mechanisms remain unsuccessful due to continous antigen stimulation, the granulomas confluate and hyalinize [15]. Cytokines may stimulate fibroblast proliferation, and these cells may also produce an increase of extracellular matrix, resulting in lung fibrosis and end-stage honeycomb lung in some patients [14].

Activated T-lymphocytes, especially CD $4^{+} \mathrm{T}$ helper (TH) 1 cells and their secretion products, as mentioned above, have been demonstrated to be increased in bronchoalveolar lavage (BAL) fluid [16,17]. 
Fig. 1. - Lymph node specimen with a noncaseating granuloma (a) (Haematoxylin \& Eosin stain, $x 250$ ). The cell-types are specified in the schematic figure $b$. (Courtesy of B. Walman)

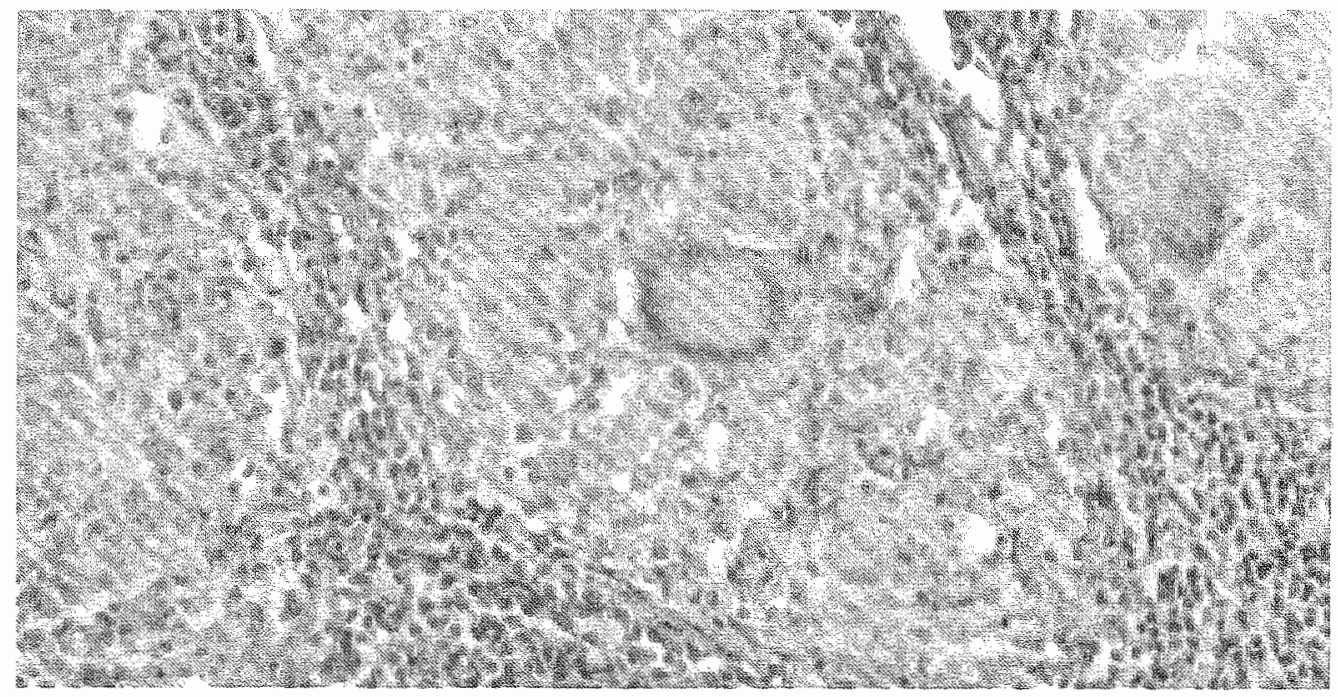

Fig. a
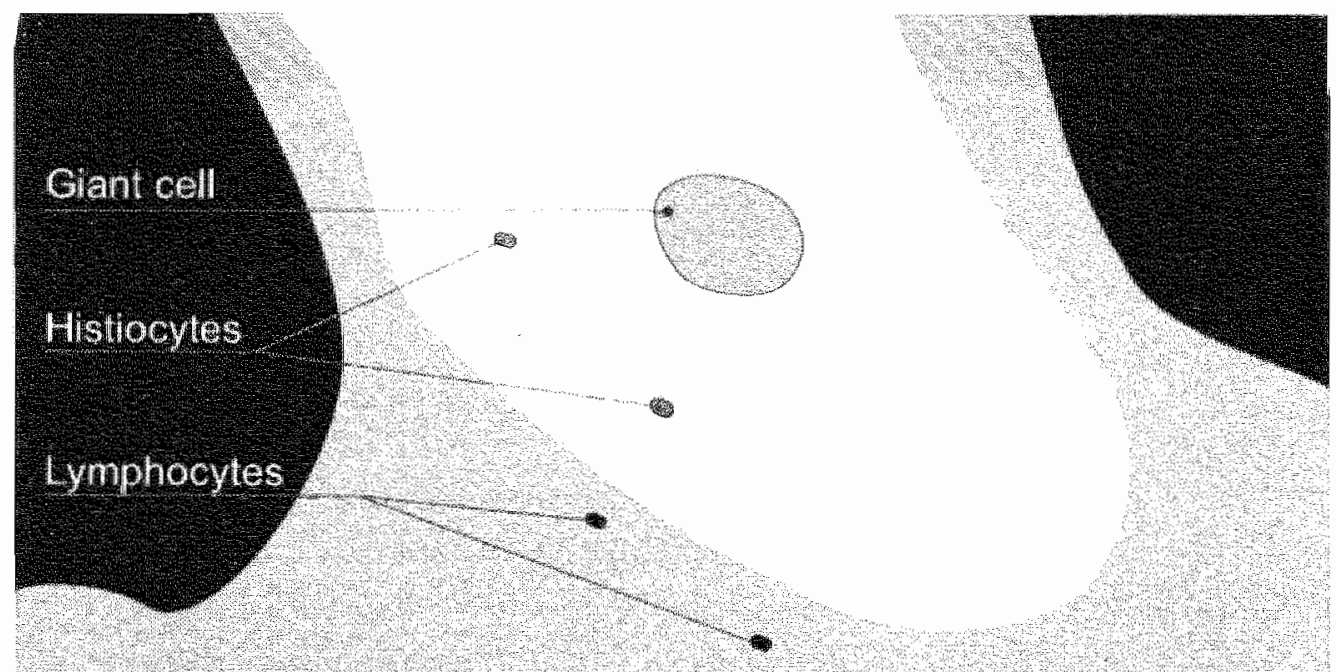

Fig. b 
Among other mediators, THI cells secrete tumour necrosis factor alpha (TNF- $\alpha)$ and interferon gamma $(\mathrm{NF}-\gamma)$. These TH1 subsets are believed to play a major role in inducing mast cell mediated immune responses such as delayed-type hypersensitivity reaction, and activating alveolar macrophages [17]. Moreover, TH1 cells are thought to promote a non-fibrotic resolution. Although pulmonary sarcoidosis is mainly associated with the expression of TH1 cytokines [18], it has been suggested that, if chronic inflammation persists, the cytokine activation may, for unknown reasons, be altered to a $\mathrm{TH} 2$ reponse [19]. These TH2 cytokines, especially interleukin (IL)-4, are thought to be responsible for the fibroblast proliferation that leads to fibrosis and honeycombing, as mentioned above [19].

Elevated levels of IL-6 and IL-8, which are released by several cell types such as monocytes/macrophages and T-lymphocytes, have been found in the BAL fluid of patients with active sarcoidosis and are thought to be important modulators in chronic inflammation because of theirnumerous proinflammatory effects $[14,20]$. However, proinflammatory cytokine production may even be found in nonactive sarcoidosis [18]. Further research is needed to address the relationship between the expression of these cytokines and disease activity [18]. Other proinflammatory mediators with presumed monocyte/macrophage origin, such as IL-1 $\beta, T N F-\alpha$ and granulocyte-macrophage-colony stimulating factor (GM-CSF), are thought to be mutually stimulatory [21]. It has been suggested that the release of these proinflammatory mediators is related to INF- $\gamma$ and IL2 [21]. The levels of INF- $\gamma,[L-1 \beta$, and TNF- $\alpha$ in inflamed sarcoid lymph nodes are higher than the serum levels in the same sarcoidosis patient [22]. The cytokines appear to be produced in the inflamed lesions of sarcoidosis and released into the bloodstream [23].

\section{Clinical features}

Pulmonary sarcoidosis is usually described as an interstitial lung disease. It is well known, however, that beside the interstitium, the bronchi of different sizes, bronchioli, and lung vessels are also involved [4]. Regardless of geography and 
race, sarcoidosis is considered to be the most common interstitial lung disease [24], but, the clinical manifestations are protean and often nonspecific. Patients with pulmonary sarcoidosis may consult a physician because of constitutional symptoms (e.g., fatigue, fever, anorexia, weight loss, a general feeling of malaise) and/or symptoms related directly to the chest, such as coughing, dyspnea on exertion, retro-sternal chest pain, chest discomfort, and wheezing $[2,24,25]$. The typical constellation of erythema nodosum, bilateral hilar lymphadenopathy, and polyarthralgia, known as Löfgren's syndrome, is found in $20-50 \%$ of patients with a more acute presentation [2].

\section{Diagnostic procedures}

The pulmonological diagnostic work-up for patients who are suspected of suffering from sarcoidosis includes radiographs and computed tomography of the chest, pulmonary function tests, bronchoscopy, including biopsies and $\mathrm{BAL}$, as well as assessment of serum markers. Moreover, extrapulmonary localisations of granulomatous inflammation should be assessed in every newly diagnosed patient [2].

The chest radiograph is rarely normal; bilateral hilar lymphadenopathy and/ or diffuse parenchymal infiltrates are the most frequent findings in patients with intrathoracic sarcoidosis. Based on the radiographic changes on first presentation, four stages have been described [26], as shown in table I.

Table I. - Radiographic stages of intrathoracic sarcoidosis *

Stage Radiographic changes

Clear chest radiograph

II Bilateral hilar lymphadenopathy

III IV Bilateral hilar lymphadenopathy and parenchymal infiltration Parenchymal infiltration without hilar lymphadenopathy Irreversible fibrosis/ bullae formation

* Adapted from O.P. Sharma [26] 
In recent years chest radiography has been complemented by computed tomography (CT) and its high resolution form (HRCT). Shotter scanning times and thinner collimations have made possible detailed demonstrations of the lung structure, and pathological changes of the lung parenchyma can be detected at earlier stages on HRCTs than on chest radiographs [27,28]. OBERSTEN et $a l$. recently suggested that HRCT can depict the inflammatory processes associated with sarcoidosis [28]. HRCT may also help to guide therapy. Ground-glass, nodular and irregular linear opacities, as well as interlobular septal thickening on HRCT suggest potentially reversible conditions, whereas findings of cystic air spaces and architectural distortion are thought to represent irreversible pulmonary disease [2,27-29].

Depending on the severity of the granulomatous inflammation in the lung, pulmonary function tests may reveal restricted lung volumes and/or impaired diffusion capacity and mildly decreased arterial oxygen pressure [24]. BAUGHMAN et al. recently showed that pulmonary function tests could be useful in predicting respiratory failure [30]. In sarcoidosis patients studied by those authors, most of those who died of respiratory failure had a vital capacity of less than 1.5 litres [30]. Abnormal pulmonary function tests are used as indicators for treatment [31]. Assessment of exercise capacity with blood gas measurements is known to be a reliable way to evaluate the degree of physiological impairment in sarcoidosis patients. In patients with a diffusion capacity of less than $50 \%$, an exercise $\mathrm{P}(\mathrm{A}-\mathrm{a}) \mathrm{O}_{2}$ much higher than the resting $\mathrm{P}(\mathrm{A}-\mathrm{a}) \mathrm{O}_{2}$ was found [32]. Recently, a higher prevalence of sleep apnea syndrome was reported in patients whose sarcoidosis was severe enough to require treatment [33]. Sarcoidosis patients with lupus pernio were found to be at higher risk of sleep apnea syndrome [33]. The higher prevalence of polysomnographic disorders in sarcoidosis patients should receive further attention.

Until recently, if the clinical presentation of a patient was indicative for the diagnosis sarcoidosis, it was strongly recommended to secure a histological confirmation $[2,5]$. However, in the past few years it has been suggested that 
such histological confirmation may not be necessary if the typical clinical features of sarcoidosis are present, such as Löfgren's syndrome together with findings indicative for sarcoidosis in the BAL fluid [16,34-37]. BAL allows recruitment of cells and solutes from the site of inflammation, the lower respiratory tract and the alveolar spaces by fibre-optic bronchoscopy [38]. BAL is a minimally invasive and safe procedure. Sterile saline is injected into a subsegmental bronchus and is subsequently aspirated and recovered. DrENT $e t$ al. showed that analysis of the BAL fluid cell profile in patients with suspected interstitial lung disease improves the diagnostic efficiency of BAL in patients suspected of having interstitial lung disease [17,39]. Increased numbers of activated, proliferating T-lymphocytes in the BAL fluid with a $\mathrm{CD} 4^{+} / \mathrm{CD} 8^{*}$ (helper/ suppressor) ratio of 4.0 or greater, reflecting a high intensity alveolitis $[40,41]$, is highly specific for sarcoidosis $[16,35]$. However, $\mathrm{CD} 4^{+} / \mathrm{CD} 8^{+}$ratios have been found to be highly variable in sarcoidosis $[36,42]$, and the number of $\mathrm{CD}^{+}{ }^{+} \mathrm{T}$ lymphocytes does not distinguish sarcoidosis from other interstitial lung diseases such as extrinsic allergic alveolitis (EAA) and idiopathic fibrosis $[36,39]$. As discussed above, the cytokines produced by the Thelper (TH) cells $\mathrm{TH} 1$ and TH2 were found to be increased in BAL fluid [21], and were suggested to be an important additional determinant in the diagnosis of interstitial lung disease [36]. Fibre-optic bronchoscopy is also used to obtain transbronchial biopsies for hisiological evidence of noncaseating granulomas. However, as discussed above, this diagnostic procedure may be necessary in only $40-50 \%$ of patients, if the diagnosis of sarcoidosis can not be confirmed by BAL [37].

Among serum markers in sarcoidosis, serum angiotensin converting enzyme (SACE) is the most widely used [31]. It is produced by epitheloid cells and alveolar macrophages and serum levels are thought to reflect the extent of granulomatous inflammation in the body $[31,43]$. However, elevated SACE levels can be found in a number of other medical conditions, e.g., in mycobacterial infections, chronic berylliosis, and chronic fatigue syndrome $[25,43]$. Its sensitivity for sarcoidosis is low $(55 \%)$, but the specificity is $99 \%$, 
given the appropriate clinical circumstances [25].

Hypercalcaemia is a well known feature with a reported incidence that varies considerably in the literature $[44,45]$. COSTABEL reports an incidence of about $5 \%$ of cases [45]. Race, age, gender, occupation, and geographic factors have not been found to influence the development of hypercalcaemia [44]. Serum calcium levels have to be assessed since persistent hypercalcaemia may lead to nephrocalcinosis and/or hypercalcaemic crisis [45]. Other serum markers, such as soluble interleukin-2 receptors ( $\mathrm{IL}-2 \mathrm{R}$ ), beta2-microglobulin, fibronectin, neopterin and others, have not reached significance in the clinical management of sarcoidosis patients [45].

Gallium-67 scanning, based on the uptake of ${ }^{67}$ Ga by activated macrophages, reflects the inflammation and the presence of granulomas $[31] .{ }^{67} \mathrm{Ga}$ scans may be of additional value in selected cases, e.g., to establish the multi-system involvement in patients with a normal chest radiograph [6], or when extrapulmonary location of granulomatous inflammation such as muscular involvement is suggested $[46,47]$.

As mentioned above, extrapulmonary location of sarcoidosis should be assessed in every newly diagnosed patient. For example, patients with suspected ophthalmic manifestations should be referred to an ophthalmologist, since eye involvement in sarcoidosis may cause permanent structural damage to the eye that may lead to blindness. Cardiac involvement is, together with progressive pulmonary fibrosis, the leading cause of death in sarcoidosis, electrocardiography should therefore be performed at diagnosis and, in cases in which cardiac involvement is suspected, during the follow-up [2]. Neurosarcoidosis, which is thought to occur in less than $10 \%$ of cases, has a predilection for the base of the brain, however, any part of the central or peripheral nervous system may be involved $[2,6]$. The diagnosis of neurosarcoidosis requires both histological confirmation of noncaseating granulomas and the clinical and radiological pictures that are compatible with sarcoidosis [6]. 


\section{Disease activity}

Disease activity in sarcoidosis should be monitored in order to provide treatment, when necessary [25]. However, the term "disease activity" has not been precisely defined and there is no general consensus on the meaning of the term [31]. The World Association of Sarcoidosis and Other Granulomatous Disorders (WASOG) consensus conference on activity in sarcoidosis stated that "the term activity implies that the disease is undergoing clinical, roentgenographical or physiological change as a consequence of the persistence of the inciting event, which of course is still unknown" [31]. There is no single test that reflects accurately disease activity [25]. In the clinical management and follow-up of sarcoidosis, clinical investigation, chest radiographs and pulmonary function tests are routinely used. SACE, ${ }^{67} \mathrm{Ga}-$ scans, and HRCT may be of additional value in selected cases [31].

\section{Course}

The course of the disease is unpredictable. The majority of patients with pulmonary sarcoidosis may be expected to undergo clinical and radiological resolution within several months to a few years [1]. Stage I on the chest radiograph is thought to have a spontaneous remission rate of $60-80 \%$, stage II about $60 \%$, and stage III less than 30\% [31]. Moreover, a correlation has been found between the mode of onset and the course and prognosis of sarcoidosis. An acute onset with erythema nodosum or asymptomatic bilateral hilar lymphadenopathy is usually self-limiting. In contrast, an insidious onset and/or extrapulmonary sarcoidosis with splenomegaly and skin lesions other than erythema nodosum, in contrast, are considered manifestations of a chronic and severe course of the disease $[5,31]$.

\section{Treatment}

Because the origin of sarcoidosis remains unknown, no causal treatment is known. The disease often resolves spontaneously and, therefore, therapeutic 


\section{Chapter 1}

intervention is not necessary in all patients [24]. There is general agreement that patients with extrapulmonary disease, such as involvement of eyes, heart or central nervous system, should be treated [9]. Moreover, as discussed above, persistent hypercalcaemia may lead to serious complications and therefore must be treated $[44,45]$. Therapy is also indicated in patients with rapid deterioration of pulmonary function tests $[2,48]$. Corticosteroids are considered the most effective therapy, they relieve symptorns, suppress the formation of gramulomas and normalize the SACE levels [5]. Although many patients with sarcoidosis respond acutely to therapy with systemic corticosteroids [48], the results of studies of their effect on the long-term outcome of pulmonary sarcoidosis are inconclusive [49-51]. Moreover, corticosteroids, as well as other immunosuppressive agents that are used as alternatives, can have significant side effects [48]. Inhaled corticosteroids are generally not recommended for the treatment of sarcoidosis [52]. However, bronchial hyperresponsiveness is assumed in about $30 \%$ of patients with sarcoidosis [53], and this may explain the positive effect of inhaled corticosteroids on respiratory symptoms [54]. Occasionally, non-steroidal anti-inflammatory drugs (NSAIDs) are prescribed to treat minimal disease [55]. These therapies may not only help to relieve arthralgia and muscle pain, but may also have an anti-inflammatory effect. In patients with advanced pulmonary sarcoidosis, lung transplantation has been successfully performed [25]. However, the development of sarcoid granulomas in the allografts has been reported and the long-term complications of recurrent sarcoidosis remain unknown $[25,56-58]$.

\section{Fatigue in sarcoidosis and some other chronic disorders}

Many patients with sarcoidosis appear to suffer from persistent constitutional symptoms such as fatigue and general weakness, even although physiological measures of disease activity may have returned to normal. JAMES suggested the existence of a "post-sarcoidosis fatigue syndrome" in patients with a history of sarcoidosis, with a normal chest radiograph and normal markers of activity of 
sarcoidosis [59]. The symptom fatigue in sarcoidosis has received little attention in the literature, whereas this symptom with respect to chronic fatigue syndrome has been studied extensively in recent years. When there is no recognized medical diagnosis to explain prolonged and disabling tatigue, together with physical and neuropsychological symptoms, chronic fatigue syndrome may be diagnosed $[60,61]$. There are controversies about the cause of chronic fatigue syndrome [62] and research has focused on the psychological as well as the somatic aspects of the syndrome [63]. As mentioned above, elevated levels of SACE have been found in patients with chronic fatigue syndrome [43], but the sACE has different characteristics from those found in sarcoidosis. The existence of a fraction of ACE, the origin of which is not known, has been suggested for chronic fatigue syndrome [43]. Evidence of immunological disturbance in patients with chronic fatigue syndrome has been reported $[64,65]$. As discussed above, in sarcoidosis an immunological disturbance is also thought to be central in the pathogenesis [66]. In both sarcoidosis and chronic fatigue syndrome, high levels of cytokines such as TNF- $\alpha$ and IL- 1 may be found $[65,22,23]$. TNF- $\alpha$ is a pro-inflammatory cytokine that plays an important role in inflammatory and immune responses [67], and a possible mediating role in relation to fatigue in inflammatory processes. TNF- $\alpha$ has indeed been found to be a critical mediator of inflammation in rheumatoid arthritis and levels of TNF- $\alpha$ have been found to be positively related to the severity of inflammation [68]. Following an infusion with anti-TNF- $\alpha$, ELLIOT et al. found that, besides an improvement in activity parameters such as C-reactive protein (CRP), joint swelling and pain, fatigue also diminished significantly in rheumatoid arthritis patients [69]. In immunemediated disorders such as sarcoidosis and others, clinical symptoms are thought to be caused partly by the effect of the accumulation of activated immunocompetent cells in the involved tissues, and partly by the increased production of different cytokines [14]. The inflammation may thus be crucial, causing the symptom fatigue in these chronic inflammatory disorders. 
In patients with chronic respiratory disorders such as chronic obstructive pulmonary disease (COPD), asthma, and restrictive lung diseases, physical sensations described in association with dyspnea include also fatigue and tiredness [70]. For patients suffering from COPD it has been shown that the exercise limitation is partly due to muscle dysfunction and that these patients experience both dyspnea as well as muscle fatigue at exercise [/1]. HAMILTON et al. found muscular weakness in respiratory muscles as well as in peripheral limb muscles in a group of patients with cardiorespiratory disorders, including obstructive and restrictive pulmonary disorders [72]. Moreover, these authors reported that muscle weakness contributed to the sensation of effort and dyspnea at exertion in these patients. In the past several years metabolic disturbances in COPD have received growing attention. Factors such as hypoxaemia, hypercapnia, malnutrition, reduced blood supply to the muscles, drugs, and electrolyte abnormalities contribute to respiratory muscle weakness and fatigue in COPD patients [73,74]. However, in sarcoidosis these factors are less likely to contribute. As in sarcoidosis, patients with COPD frequently suffer from chronic inflammation contributing to the high percentage of weight loss and depletion of fat free mass in this patient population [75]. Beside these effects, the direct role of inflammatory products on different aspects of muscle metabolism has been emphasized. In their work in hamsters, Wllcox et al. demonstrated that monocyte secretory products impair diaphragmatic contractility in vitro [76]. Moreover, impaired contractility of the respiratory muscles was demonstrated in animal models with sepsis. They assumed that a reduction in the contractility of the diaphragm was mediated by inflammatory products released during sepsis [76]. Which factor or combination of factors is responsible for this effect is not known. Identification of these products could lead to specific therapeutic interventions aimed at blocking their effects in patients with inflammatory disorders such as sarcoidosis. 
Psychological aspects of sarcoidosis

Various aspects of sarcoidosis mentioned above, such as the relatively young age at diagnosis, the need to adjust to a potentially life-long chronic disease, the unpredictable course of the disease, and the broad range of frequently persistent symptoms may lead to psychosocial problems. In addition, the patients' lack of knowledge of the consequences of sarcoidosis and the disease itself may give rise to problems in their work and social life. In the sarcoidosis literature these aspects have received little attention. Two studies have addressed the psychollogical perspective of the disease. First, KLONOFF and KLEINHENZ reported a relationship between life stress and impairment of lung function tests [77]. The authors pointed out that although patients did not meet the clinical criteria for psychiatric diagnoses, the clinical picture with anxiety, depression, and vague physical symptoms could lead to misdiagnosis as a somatization disorder. Second, the psychosomatic aspects of sarcoidosis were studied by LANGE et al. [78], who found that higher levels of anxiety and depression could only partly be interpreted as reactive to the disease. Levels of anxiety and depression did not change significantly over time and were independent of the course of the disease. Patients with a "depressive personality" who had suffered difficult life situations before becoming ill had a greater chance of developing a "subacutechronic" or a progressive chronic course of the disease [78]. However, as stressed by these authors, prospective studies of psychosomatic factors in sarcoidosis can not be performed, since it remains a relatively rare disorder, and if the disease is asymptomatic it may not be diagnosed at all. Retrospective studies, may also be difficult to interpret, since reaction to the disease can not be separated from premorbid traits [78]. 


\section{Quality of life research}

The impact of disease on patients' lives can be evaluated by assessing their health status and quality of life (QOL), which have become important topics in health care over the past two decades. Health status and QOL are now accepted as important outcome measures [79] and can add to medical information, e.g., in clinical trials [80]. Moreover, the centrality of the patient's point of view in monitoring medical care outcomes has been recognized [81]. Most research in QOL is related to health, although the term health-related QOL has not been defined explicitly, as noted by De Haes and VAN KNIPPENBERG as long ago as 1985 [82], and this remark still holds today. The terms health status, healthrelated QOL, and QOL are used in a confusing way in the medical literature and are often not clearly distinguished. In contrast with broad QOL instruments, health-related QOL instruments focus only on health-related issues. Health status measures assess mainly functional status, whereas QOL instruments are necessarily subjective. Measures that assess health status often claim to evaluate QOL [83]. One problem when using health status measures is that lower levels of functioning may be equated with lower QOL. This contrasts with findings in QOL research reflecting high perceived QOL in spite of low levels of functioning [84]. In contrast with health status, QOL is subjective and was defined by the World Health Organization Quality of Life group [85] as "an individual's perception of his/her position in life in the context of the culture and value systems in which he/she lives and in relation to his/her goals, expectations, standards and concerns". This is a broad concept, incorporating in a complex way the individual's physical health, psychological state, level of independence, social relationships, personal beliefs, and their relationship to salient features of the environment [85]. This definition highlights the view that QOL is subjective, that it includes both positive and negative facets of life, and is multi-dimensional. 
The existing health status and QOL measures can be divided into generic and disease-specific questionnaires. Generic measures are designed to assess health status or QOL across different types and severities of diseases and patient groups [86]. In contrast, disease-specific instruments are designed for the assessment of specific illnesses and patient populations [86]. There are also domain-specific measures for certain functions such as dyspnea or mental health [86].

The Sickness Impact Profile (SIP) [87] is one of the most broadly used generic health status measures. It was designed to assess sickness-related physical and psychosocial dysfunction and behavioural changes, as well as to detect changes or differences over time or between groups [87]. Measures like the SIP focus on the impact of disease on an individual's physiological, psychological, and/or social functioning. These three aspects have formed part of most health status instruments since they were included in the definition of health by the World Health Organization in 1958 [88]. A recently developed generic QOL measure, the World Health Organization Quality of Life assessment instrument (WHOQOL-100) assesses the individual's perception and includes aspects of life linked directly as well as indirectly to health [85]. The WHOQOL-100 consists of 100 items assessing 24 facets of QOL within six domains (physical health, psychological health, level of independence, social relationships, environment, and spirituality/religion/personal beliefs) and a general evaluative facet (overall quality of life and general health). It was developed cross culturally and simultaneously in 15 centers around the world, including, France, Russia, the USA, Panama, Zimbabwe, Japan, Thailand, and The Netherlands [85].

Over the past two decades, using generic instruments, comparative studies have been performed on health status for a considerable number of chronic diseases such as end-stage renal disease, rheumatoid arthritis, chronic obstructive pulmonary disease (COPD), angina, and chronic low back pain [86]. Employing the SIP, the health status of rheumatoid arthritis patients was found 
to be slightly better than in non-oxygen dependent COPD patients [86]. For patients with Crohn's disease, a chronic inflammatory disorder characterized by granuloma formation, their health status was somewhat better than for those with either rheumatoid arthritis or COPD [86]. For asthma [89] and COPD, disease-specific health status instruments have been developed [90-92], some of which have also been translated into and validated for several languages [93,94]. COPD has been found to be associated with impaired health status and "QOL" [95-97], as well as with higher incidences of depression [96-98] and anxiety [98]. The relationship between these findings and the physiological parameters of the severity of COPD, however, was found to be weak [99-101].

For sarcoidosis, as for other interstitial lung diseases, no studies of health status, health-related QOL, or QOL have been undertaken [102]. One reason for this may be that, in comparison with other inflammatory disorders such as rheumatoid arthritis and other respiratory diseases such as COPD and asthma, sarcoidosis remains a relatively rare disorder. However, considering some of the aspects mentioned above, assessment of health status and QOL in sarcoidosis may help to offer better care to this patient group, especially to those with a chronic course of the disease. In addition, markers are needed for the objective assessment of frequently persistent general symptoms such as fatigue, general weakness, and reduced exercise capacity in sarcoidosis patients. 


\section{References}

1. James DG. Clinical picture of sarcoidosis. In: Schwarz MI, King TE, eds. Interstitial Lung Disease. Mosby-Year Book, St. Louis, Missouri 1993; 159178.

2. Newman LS, Rose CS, Maier LA. Sarcoidosis. N Engl J Med 1997; 336: 1224 1234.

3. Mitchell DN, Scadding JG. Sarcoidosis. Am Rev Respir Dis 1974; 110: 774 802.

4. Thomas PD, Hunninghake GW. Current concepts of the pathogenesis of sarcoidosis. Am Rev Respir Dis 1987; 135: 747-760.

5. Yamamoto M, Sharma OP, Hosada Y. The 1991 descriptive definition of sarcoidosis. Sarcoidosis 1992; 9 (suppl 1): 33-34.

6. Sharma OP. Neurosarcoidosis. A personal perspective based on the study of 37 patients. Chest $1997 ; 112: 220-228$.

7. James DG. Historical background $I n$ : James GD, ed. Sarcoidosis and Other Granulomatous Disorders. New York, Dekker, 1994; 1-18.

8. James DG. Epidemiology of sarcoidosis. Sarcoidosis 1992; 9: 79-87.

9. du Bois RM: Sarcoidosis. In: Walters EH, du Bois RM, eds. Immunology and Management of Interstitial Lung Diseases. Chapman \& Hall, London, 1995; $97-122$

10. Hillerdal G, Nöu E, Osterman K, Schmekel B. Sarcoidosis: epidemiology and prognosis. A 15-year European study. Am Rev Respir Dis 1984; 130: 29-32.

11. Fité E, Alsina JM, Mañá J, Pujol R, Ruiz J, Morera J. Epidemiology of sarcoidosis in Catalonia: 1979-1989. Sarcoidosis 1996; 13: 153-158.

12. Pietinalho A, Ohmichi M, Hiraga Y, Löfroos A-B, Selroos O. The mode of presentation of sarcoidosis in Finland and Hokkaido, Japan. A comparative analysis of 571 Finnish and 686 Japanese patients. Sarcoidosis 1996; 13:159166.

13. Harrington DW, Major M, Rybicki B, Popovich J, Maliarik M, Iannuzzi MC. Familial sarcoidosis: analysis of 91 families. Sarcoidosis $1994 ; 11: 240-243$.

14. Semenzato G, Agostini C. Immunology of sarcoidosis. In: Schwarz MI, King TE eds. Interstitial Lung Disease. Mosby-Year Book, St. Louis, Missouri 1993 ; 127-158.

15. Mitchell DN, Scadding JG, Heard BE, Hinson KFW. Sarcoidosis: histopathological definition and clinical diagnosis. J Clin Path 1977; 30: 395408

16. Winterbauer RH, Lammert J, Selland M, Wu R, Corley D, Springmeyer SC. Bronchoalveolar lavage cell populations in the diagnosis of sarcoidosis. Chest 1993; 104: 352-361.

17. Drent M, Mulder PGH, Wagenaar SiSc, Hoogsteden HC, Van Velzen-Blad. H, Van den Bosch JMM. Differences in BAL fluid variables in interstitial lung diseases evaluated by discriminant analysis. Eur Respir J1993; 6: 803-810. 
18. Minshall EM, Tsicopoulos A, Yasruel Z, et al. Cytokine mRNA gene expression in active and nonactive pulmonary sarcoidosis. Eur Respir J1997; 10:2034-2039.

19. Kunkel SL, Lukacs NW, Strieter RM, Chensue SW. Th1 and Th2 responses regulate experimental lung granuloma development. Sarcoidosis Vasc Diffuse Lung Dis 1996; 13: 120-128.

20. Girgis RE, Basha MA, Maliarik M, Popovich J, Jr, Iannuzzi MC. Cytokines in the bronchoalveolar lavage fluid of patients with active pulmonary sarcoidosis. Am J Respir Crit Care Med 1995; 152: 71-75.

21. Prior C, Knight RA, Herold M, Ott G, Spiteri MA. Pulmonary sarcoidosis: patterns of cytokine release in vitro. Eur Respir $J 1996 ; 9: 47-53$.

22. Shijubo N, Imai K, Shigehara K, et al. Soluble intercellular adhesion moleculeI (ICAM-1) in sera and bronchoalveolar lavage fluid of patients with idiopathic pulmonary fibrosis and pulmonary sarcoidosis. Clin Exp Immunol 1994; 95 : $156-161$.

23. Asano M, Minagawa $T$, Ohmichi M, HiragaY. Detection of endogenous cytokines in sera or in lymph nodes obtained from patients with sarcoidosis. Clin Exp Immunol 1991; 84: 92-96.

24. Müller-Quernheim J. The cytokine network in sarcoidosis. Eur Cytokine Netw $1996 ; 7: 13-26$.

25. Sharma OP, Alam S. Diagnosis, pathogenesis, and treatment of sarcoidosis. Curr Opin Pulm Med 1995; 1: 392-400.

26. Sharma OP. Pulmonary sarcoidosis: radiographic features. In: James GD, ed. Sarcoidosis and Other Granulomatous Disorders. New York, Dekker, 1994; 213-245.

27. Remy-Jardin MC, Beuscart R, Sault M, Marquette CH, Remy J. Subpleural micronodules in diffuse infiltrative lung disease: evaluation with thin-section CT scans. Radiology 1990; 177: 133-139.

28. Oberstein A, von Zitzewitz F, Schweden F, Müller-Quernheim J. Non invasive evaluation of the inflammatory activity in sarcoidosis with high-resolution computed tomography. Sarcoidosis Vasc Diffuse Lung Dis 1997; 14: 65-72.

29. Murdoch J, Müller NL. Pulmonary sarcoidosis: changes on follow-up CT examination. AJR 1992; 159: 473-477.

30. Baughman RP, Winget DB, Bowen EH, Lower EE. Predicting respiratory failure in sarcoidosis patients. Sarcoidosis Vasc Diffuse Lung Dis 1997; 14:

31. Costabel U, du Bois RM, Eklund A et al. Consensus conference: activity of sarcoidosis. Third WASOG meeting, LoS Angeles, USA, September 8-11,
1993. Eur Respir J 1994; 7:624-627.

32. Badr Ar, Sharma OP. Pulmonary function. I $m$ : James GD, ed. Sarcoidosis and Other Granulomatous Disorders. New York, Dekker, 1994; 247-266.

33. Tumer GA, Lower EE, Corser BC, Gunther KL, Baughman RP. Sleep apnea in sarcoidosis. Sarcoidosis Vase Diffuse Lung Dis 1997; 14:61-64.

34. Costabel U, Zaiss AW, Guzman J. Sensitivity and specificity of BAL findings in sarcoidosis. Sarcoidosis 1992; 9 (suppl 1): 211-214. 
35. Thomeer $M$, Demedts $M$. Predictive value of $\mathrm{CD} 4 / \mathrm{CD} 8$ ratio in bronchoalveolar lavage in the diagnosis of sarcoidosis. Sarcoidosis Vasc Diffuse Lung Dis 1997; 14 (suppl 1): 36.

36. Drent M, Grutters JC, Mulder PGH, VanVelzen-Blad H, Wouters EFM, Van den Bosch JMM. Is the different $T$ helper cell activity in sarcoidosis and extrinsic allergic alveolitis also reflected by the cellular bronchoalveolar lavage fluid profile? Sarcoidosis Vasc Diffuse Lung Dis 1997; 14:31-38.

37. Costabel U. CD4/CD8 ratios in bronchoalveolar lavage fluid: of value for diagnosing sarcoidosis? Eur Respir J 1997; 10:2699-2700.

38. Eklund A, Grunewald J. The riddle of sarcoidosis: have novel techniques brought any new insights as to the causative agent? J Int Med 1996;240:59-62.

39. Drent M, Van Nierop MAMF, Gerritsen FA, Wouters EFM, Mulder PGH. A computer program using BALF-analysis results as a diagnostic tool in interstitial lung diseases. Am J Respir Crit Care Med 1996; 153: 736-741.

40. Verstraeten A, Demedts $\mathrm{M}$, Verwilghen J, et al. Predictive value of bronchoalveolar lavage in pulmonary sarcoidosis. Chest 1990; 98:560-567.

41. Drent M, Van Velzen-Blad H, Diamant M, Hoogsteden $\mathrm{HC}_{\text {, Van den Bosch }}$ JMM. Relationship between presentation of sarcoidosis and $\mathrm{T}$ lymphocyte profile: a study in bronchoalveolar fluid. Chest 1993; 104: 795-800.

42. Kantrow SP, Meyer KC, Kidd P, Raghu G. CD4/CD8 ratio in BAL fluid is highly variable in sarcoidosis. Eur Respir J 1997; 10: 2716-2721.

43. Lieberman J, Bell DS. Serum angiotensin-converting enzyme as a marker for the chronic fatigue-immune dysfunction syndrome: a comparison to serum angiotensin-converting enzyme in sarcoidosis. Am J Med 1993; 95: 407-412.

44. Sharma OP. Vitamin D, calcium, and sarcoidosis. Chest 1996; 109: 535-539.

45. Costabel U, Teschler H. Biochemical changes in sarcoidosis. Clin Chest Med $1997 ; 18: 827-842$.

46. Edan G, Bourguet P, Delaval Ph, Herry JY. Gallium-67 imaging in muscular sarcoidosis. J Nucl Med 1984; 25:776-778.

47. Liem IH, Drent $M_{3}$ Antevska E, Lamers RJS, Heidendal GAK. Intensive muscle uptake of Gallium-67 in a patient with sarcoidosis. J Nucl Med 1998; 39: 1605 1607.

48. Hunninghake $\mathrm{GW}$, Gilbert $\mathrm{S}$, Pueringer $\mathrm{R}$, et al. Outcome of the treatment for sarcoidosis. Am J Respir Crit Care Med 1994; 149: 893-898.

49. Gibson GJ, Prescott RJ, Muers MF, et al. British Thoracic Society Sarcoidosis study: effects of long term corticosteroid treatment. Thorax. 1996; 51:238-247.

50. Gottlieb JE, Israel HL, Steiner RM, Triolo J, Patrick H. Outcome in sarcoidosis. The relationship of relapse to corticosteroid therapy. Chest 1997; 111:623-631.

51. Eule H, Weinecke A, Roth I. The possible influence of corticosteroid therapy on the natural course of pulmonary sarcoidosis. Ann NY Acad Sci 1986; 465: 695701.

52. Kirsten D. Inhaled steroids for sarcoidosis? Editorial. Eur Respir J 1995; 8: 679681.

53. Kirsten D, Nowak D, Pielesch W, Magnussen $H$. Hyperreaktivităt und obstruktive Ventillationsstörung bei Lungensarkoidose. Pneumologie 1992; 46: 278-279 
54. Dutch Study Group on Pullmonary Sarcoidosis, Alberts C, Van der Mark ThW, Jansen HM. Inhaled budesonide in pulmonary sarcoidosis: a double-blind, placebo-controlled study. Eur Respir J 1995; 8: 682-688.

55. Baughinan RP, Lower EE. Alternatives to corticosteroids in the treatment of sarcoidosis. Sarcoidosis Vasc Diffuse Lung Dis 1997; 14: 121 -130.

56. Martinez FJ, Orens JB, Deeb M, Brunsting LA, Flint A, Lynch JP, III. Recurrence of sarcoidosis following bilateral allogeneic lung transplantation. Chest 1994; 106: 1597-1599.

57. Walker $\mathrm{S}$, Mikhail $\mathrm{G}$, Banner $\mathrm{N}$, et al. Medium term results of lung transplantation for end stage pulmonary sarcoidosis. Thorax 1998; 53:281-284.

58. Judson MA. Lung transplantation for pulmonary sarcoidosis. Eur Respir $J$ 1998; 11: 738-744.

59. James DG. Complications of sarcoidosis. Chronic fatigue syndrome. Sarcoidosis 1993; 10:1-3.

60. Archer MI. The post-viral syndrome: a review. J Gen Pract 1987; 37:212-214.

61. Holmes GP, Kaplan JE, Gantz NM et al. Chronic fatigue syndrome: a working case definition. Ann Intern Med 1988; 108: 387-389.

62. Masuda A, Nozoe S-I, Matsuyama T, Tanaka H. Psychobehavioral and immunological characteristics of adult people with chronic fatigue and chronic fatigue syndrome. Psychosom Med 1994; 56: 512-518.

63. Ray C, Jefferies S, Weir WRC. Life-events and the course of chronic fatigue syndrome. Brit J Med Psychol 1995; 68: 323-331.

64. Landay AL, Jessop C, Lennette ET, Levy JA. Chronic fatigue syndrome: clinical condition associated with immune activation. Lancet 1991; 338: 707 712 .

65. Patarca $\mathrm{R}$, Klimas $\mathrm{NG}$, Lugtendorf $\mathrm{S}$, Antoni M, Fletcher MA. Dysregulated expression of tumor necrosis factor in chronic fatigue syndrome: interrelations with cellular sources of soluble immune mediator expression. Clin Infect Dis 1994; 18 (supp 1): S147-S153.

66. Semenzato $G$, Zambello $R$, Trentin L, Agostini C. Cellular immunity in sarcoidosis and hypersensitivity pneumonitis. Recent advances. Chest 1993; 103 (suppl): 139S-143S.

67. Jäăttelä. M. Biologic activities and mechanisms of action of tumor necrosis factor- alpha/cachectin. Lab Invest 1991; 64: 724-741.

68. Saxne T, Palladino MA Jr, Heinegărd D, Talal N, Wollheim FA. Detection of tumor necrosis factor $\alpha$ but not tumor necrosis factor $\beta$ in rheumatoid arthritis synovial fluid and serum. Arthritis Rheum 1988; 31: 1041-1045.

69. Elliott MJ, Maini RN, Feldmann $M$, et al. Randomised double-blind comparison of chimeric monoclonal antibody to tumour necrosis factor $\alpha$ (cA2) versus placebo in rheumatoid arthritis. Lancet 1994; 344: 1105-1110.

70. Janson-Bjerklie S, Kohlmann Carrieri V, Hudes M. The sensation of pulmonary dyspnea. Nurs Res 1986; 35: 154-159.

71. Killian KJ, LeBlanc P, Martin DH, Summers E, Jones NL, Campbell EJ. Exercise capacity and ventilatory, circulatory, and symptom limitation in patients with chronic airflow limitation. Am Rev Respir Dis 1992; 146:935-940. 
72. Hamilton AL, Killian KJ, Summers E, Jones NL, Muscle strength, symptom intensity, and exercise capacity in patients with cardiorespiratory disorders. Am J Respir Crit Care Med 1995; 152: 2021-2031.

73. Dekhuijzen PNR, Gayan-Ramirez G, Decramer M. Does corticosteroid treatment affect the respiratory muscles? Eur Respir J 1993; 6: 465-466.

74. Tobin MJ. Respiratory muscles in disease. Clin Chest Med 1988; 9:263-286.

75. Schols AMWJ, Soeters PB, Dingemans AMC, Mostert R, Frantzen PI, Wouters EFM. Prevalence and characteristics of nutritional depletion in patients with stable COPD eligible for pulmonary rehabilitation. Am Rev Respir Dis 1993; 147: 1151-1156.

76. Wilcox $\mathrm{O}$, Osborne $\mathrm{S}$, Bressler $\mathrm{B}$. Monocyte inflammatory mediators impair in vitro hamster diaphragm contractility. Am Rev Respir Dis 1992; 146:462-466.

77. Klonoff EA, Kleinhenz ME. Psychological factors in sarcoidosis: the relationship between life stress and pulmonary function. Sarcoidosis 1993; 10 : 118-124.

78. Lange C, Schüßler G, Hüttemann U. Psychosomatische Aspekte bei der Sarkoidose. Pneumologie 1995; 49: 14-19.

79. Hays RD, Shapiro MF. An overview of generic helath-related quality of life measures for HIV research. Quality of Life Research 1992; 1:91-97.

80. Moinpour CM, Feigl P, Metch B, Hayden KA, Meyskens FL, Jr, Crowlley J. Quality of life end points in cancer clinical trials: review and recommendations. J Natl Cancer Inst 1989; 81: 485-495.

81. Ware JE, Sherbourne CD. The MOS 36-item Short-Form Heallh Survey (SF36): I. Conceptual framework and item selection. Med Care 1992; 30:473-483.

82. De Haes JCJM, Van Knippenberg FCE. The quality of life of cancer patients: a review of the literature. Soc Sci Med 1985; 20: 809-817.

83. De Vries J. Beyond health status. Construction and walidation of the Dutch WHO Quality of Life assessment instrument. Doctoral thesis, Tilburg University, Tilburg, The Netherlands, 1996.

84. O'Boyle CA. The schedule for the evaluation of individual quality of life (SEIQoL). Int J Ment Health 1994; 23: 3-23.

85. WHOQOL group. Development of the WHOQOL: rationale and current status. Int J Ment Health 1994; 23: 24-56.

86. Patrick DL, Deyo RA. Generic and disease-specific measures in assessing health status and quality of life. Med Care 1989;27: S217-S232.

87. Bergner M, Bobbitt RA, Carter WB, Gilson BS. The Sickness Impact Profile: development and final revision of a health status measure. Med Care 1981; 19: 787-805.

88. WHO. The first ten years of the World Health Organization 1958; Geneva: WHO.

89. Juniper EF, Guyatt GH, Epstein RS, Ferrie PJ, Jaeschke R, Hiller TK. Evaluation of impairment of health related quality of life in asthma: development of a questionnaire for use in clinical trials. Thorax 1992; 47: 7683. 
90. Guyatt GH, Berman LB, Townsend M, Pugsley SO, Chambers LW. A measure of quality of life for clinical trials in chronic lung disease. Thorax 1987; 42: 773 778.

91. Jones PW, Quirk FH, Baveystock CM, Littlejohns P. A self-complete measure of health status for chronic airflow limitation: the St. George's Respiratory Questionnaire. Am Rev Respir Dis 1992; 145: 1321-1327.

92. Maillé AR, Koning CJM, Zwinderman AH, Willems LNA, Dijkman JH, Kaptein AA. The development of the 'Quality-of-Life for Respiratory Illness Questionnaire (QOL-RIQ)': a disease-specific quality-of-life questionnaire for patients with mild to moderate chronic nonspecific lung disease. Respir Med 1997; 91: 297-309.

93. Engström C-P, Persson L-O, Larsson S, Sullivan M. Reliability and validity of a Swedish version of the St George's Respiratory Questionnaire. Eur Respir J 1998; 11:61-66

94. Güell R, Casan P, Sangenis M, Morante F, Belda J, Guyatt GH. Quality of life in patients with chronic respiratory disease: the Spanish version of the Chronic Respiratory Questionnaire (CRQ). Eur Respir $J$ 1998; 11:55-60.

95. Guyatt GH, Townsend M, Berman LB, Pugsley SO. Quality of life in patients with chronic airflow limitation. Br J Dis Chest 1987; 81 : 45-54.

96. McSweeny AJ, Grant I, Heaton RK, Adams KM, Timms RM. Life quality of patients with chronic obstructive pulmonary disease. Arch Intern Med 1982; 142: 473-478.

97. Dudley DL, Glaser EM, Jorgenson BN, Logan DL. Psychosocial concomitants to rehabilitation in chronic obstructive pulmonary disease. Part I. Psychosocial and psychological considerations. Chest 1980; 77: 413-420.

98. Light RW, Merrill EJ, Despars JA, Gordon GH, Mutalipassi LR. Prevalence of depression and anxiety in patients with COPD. Relationship to functional capacity. Chest 1985; 87: 35-38.

99. Schrier AC, Dekker FW, Kaptein AA, Dijkman JH. Quality of life in elderly patients with chronic nonspecific lung disease seen in family practice. Chest 1990; 98: 894-899.

100. Williams SJ, Bury MR. Impairment, disability and handicap in chronic respiratory illness. Soc Sci Med 1989; 29: 609-616.

101. Curtis JR, Deyo RA, Hudson LD. Health-related quality of life among patients with chronic obstructive pulmonary disease. Thorax 1994; 49: 162-170.

102. Bouros D, Psathakis K, Siafakas NM. Quality of life in interstitial lung disease. Eur Respir Rev 1997; 7: 42, 66-70. 
Chapter 2

Aims of the study 


\section{AIMS OF THE STUDY}

The aims of this study were to evaluate the impact of sarcoidosis on patients' health status and QOL. Moreover, a possible relationship between these measures and physiological parameters such as chest radiograph, serum angiotensin converting enzyme ( $\mathrm{SACE}$ ), and pulmonary function tests was studied. Another important aspect of the present study was the analysis of the frequency of nonspecific constitutional symptoms such as fatigue and general weakness in sarcoidosis patients and their relationship with physiological measures. Until now the physiological basis of these symptoms in sarcoidosis is unknown and no objective measures for the assessment of them exist.

For the assessment of health status or quality of life (QOL), either generic or disease-specific instruments can be employed. For other respiratory diseases such as asthma and COPD disease-specific health status measures have been developed [1-3]. Although the lung is the most frequently affected organ in sarcoidosis, considering the disseminated character of the disease and the broad range of possible symptoms, sarcoidosis is only partly comparable with other respiratory disorders. Therefore, a generic health status measure, the Sickness Impact Profile (SIP) [4] and a generic QOL instrument, the World Health Organization Quality of Life assessment instrument (WHOQOL-100) [5], were employed.

Three different patient populations were recruited. The first consisted of patient members ( $\mathrm{n}=1026)$ of the Dutch Sarcoidosis Society. This group was studied with the main object to obtain a picture of the clinical presentation of sarcoidosis in the Netherlands. The second study population $(n=64)$ was recruited from the departments of pulmonology of eight Dutch Hospitals. In these out-patients, current symptoms, serum angiotensin converting enzyme (SACE), pulmonary function tests, and chest radiographs were evaluated. 
Moreover, these patients completed various questionnaires in order to assess health status, QOL, and depressive symptoms. The third patient group consisted of consecutive out-patients $(n=38)$ with recently diagnosed sarcoidosis, recruited from the Department of Pulmonology of the Academic Hospital Maastricht. In these patients besides a broad range of other physiological parameters, metabolic measures were assessed.

In Chapter 3 the results of the survey among the members of the Dutch Sarcoidosis Society is presented. The aim of this study was to evaluate present symptoms and symptoms at the time of the onset of the disease as well as family history, diagnostic procedures, diagnostic delay, and treatment in the Dutch sarcoidosis patient population.

In Chapter 4 health status of the out-patients recruited from the pulmonary departments of the participating hospitals $(n=64)$ was assessed, using a general health status measure, the Sickness Impact Profile (SIP) [4]. As fatigue is a major symptom in clinical depression as well, depressive symptoms in sarcoidosis patients were assessed using the Beck Depression Inventory (BDI) [6]. In addition, the relationship between health status and depressive symptoms was studied.

Since fatigue appeared to be the most frequently reported symptom in sarcoidosis, in Chapter 5 we assessed the QOL of sarcoidosis patients, using a broad ranging QOL instrument, the WHOQOL-100 [5], which also measures fatigue. QOL was studied separately in sarcoidosis patients suffering from current symptoms and in patients who reported no symptoms at the time the study was conducted. These groups were compared with each other as well as with a healthy control group. Moreover, the relationship between the psychological health of these patients and fatigue as well as sleeping problems, was studied.

In contrast to sarcoidosis, QOL and health status have been studied extensively in other chronic diseases such as rheumatoid arthritis (RA) [7]. Since sarcoidosis and RA both are chronic inflammatory disorders and have a 
number of clinical features in common, in Chapter 6 we compared QOL in these two disorders using the WHOQOL-100 [5].

Involvement of muscles in sarcoidosis has been reported in $50-80 \%$ of cases [8]. Muscle weakness of respiratory muscles as well as of the peripheral muscles was associated with the sensation of effort and dyspnea on exertion in patients with cardiorespiratory disorders [9]. Therefore, in Chapter 7 the relationships between pulmonary function tests, respiratory muscle strength and endurance time, as well as health status assessed by the SIP [4] were studied in a subgroup $(n=18)$ of the study population, recruited from the Department of Pulmonology of the Academic Hospital Nijmegen St. Radboud.

QOL measures such as the WHOQOL-100 [5] are able to assess fatigue subjectively in sarcoidosis. However, markers to objectify nonspecific constitutional symptoms in sarcoidosis, such as fatigue and general weakness, are needed. It is tempting to speculate that in sarcoidosis, as in other inflammatory conditions, metabolic alterations are involved. In Chapter 8 a broad range of physiological variables, e.g., C-reactive protein (CRP), sACE, pulmonary function tests, as well as metabolic measures such as body composition and resting energy expenditure were assessed. In 38 patients recruited from the Academic Hospital Maastricht the relationship between these measures and the most common symptom in sarcoidosis, e.g, fatigue, was studied. Chapter 9 contains a summary, a general discussion, and the impact of the study on future research in sarcoidosis. 


\section{References}

1. Juniper EF, Guyatt GH, Epstein RS, Ferrie PI, Jaeschke R, Hiller TK. Evaluation of impairment of health related quality of life in asthma: development of a questionnaire for use in clinical trials. Thorax $1992 ; 47: 76-83$.

2. Jones PW, Quirk FH, Baveystock CM, Littlejohns P. A self-complete measure of health status for chronic airflow limitation: the St. George's Respiratory Questionnaire. Am Rev Respir Dis 1992; 145: 1321-1327.

3. Maillé AR, Koning CJM, Zwinderman AH, Willems LNA, Dijkman JH, Kaptein AA. The development of the 'Quality-of-Life for Respiratory Illness Questionnaire (QOL-RIQ)': a disease-specific quality-of-life questionnaire for patients with mild to moderate chronic non-specific lung disease. Respir Med 1997; 91: 297309.

4. Bergner M, Bobbitt RA, Carter WB, Gilson BS. The Sickness Impact Profile: development and final revision of a health status measure. Med Care 1981; 19: 787-805.

5. WHOQOL group. Development of the WHOQOL: rationale and current status. Int JMent Health 1994; 23: 24-56.

6. Beck AT, Ward CH, Mendelson M, Mock J, Erbaugh J. An inventory for measuring depression. Arch Gen Psychiat 1961; 4: 561-571.

7. Van Riel P, Van Lankveld W. Quality of life in rheumatoid arthritis: a review, Pharm World Sci 1993; 15:93-97.

8. Silverstein A, Siltzbach LE. Muscle involvement in sarcoidosis: asymptomatic, myositis, and myopathy. Arch Neurol 1969;21:235-241.

9. Hamilton AL, Killian KJ, Summers E, Jones NL. Muscle strength, symptom intensity, and exercise capacity in patients with cardiorespiratory disorders. $A m J$ Respir Crit Care Med 1995; 152: 2021-2031. 
Chapter 3

Clinical presentation of sarcoidosis in the Netherlands. An epimediological study 


\section{Clinical presentation of sarcoidosis in the Netherlands. An epidemiological study}

R.M. Wirnsberger*, J. de Vries**, E.F.M. Wouters*, M. Drent*

*Department of Pulmonology, University Hospital Maastricht, **Department of Psychology, Tilburg University, The Netherlands

Neth J Med 1998; 53: 53-60 


\section{Abstract}

Patients suffering from sarcoidosis may present with a wide range of symptoms. The aim of this study was to make an inventory of the clinical presentation of the sarcoidosis population in the Netherlands.

Symptom inventory questionnaires were sent to all members of the Dutch Sarcoidosis Society. Of these 1755 sarcoidosis patients, 1026 (58\%), (age $46.7 \pm 11.6$, female $63 \%$ ) completed the questionnaire.

Familial sarcoidosis was reported by 170 patients (16.3\%). In $57 \%$ of the cases the first diagnosis was sarcoidosis. Other diagnoses included rheumatoid arthritis $(5.1 \%)$ and tuberculosis ( $4.8 \%)$. Treatment with systemic corticosteroids was reported by 565 patients $(55.1 \%)$. The most frequently reported symptom was fatigue ( $71 \%$ ), followed by dyspnea (70\%), arthralgia (52\%), muscle pain $(39 \%)$, chest pain (27\%), and general weakness (22\%). Moreover, $26 \%$ of patients suffered from disease-related tension and strain. No relationship was found between the reported symptoms and treatment with corticosteroids.

Sarcoidosis patients suffered from a broad range of persistent physical symptoms. In this study fatigue appeared to be the most commonly reported symptom. Intervention programs should focus on physical health as well as psychosocial aspects such as teaching patients how to cope with the disease.

\section{Introduction}

In sarcoidosis, a disseminated granulomatous disease of unknown origin, practically every organ can be involved [1]. Depending on the organs involved and the severity of granulomatous inflammation, symptoms can vary considerably. Besides respiratory symptoms such as coughing and dyspnea on exertion, patients often suffer from systemic symptoms such as fever, weight loss, and fatigue $[2,3]$. 
In the follow-up of sarcoidosis patients, routine tests to assess disease activity include clinical investigation, chest radiography, and lung function testing [4]. Serum angiotensin converting enzyme (SACE), gallium-67 scanning, high resolution computed tomography, and bronchoalveolar lavage parameters may also be useful as indicators of disease activity [4], although they do not necessarily reflect well-being of the patient. For example, even if pulmonary function tests, SACE, and radiographic abnormalities return to normal or show only small impairment, nonspecific symptoms, such as fatigue and reduced exercise capacity may persist.

In other chronic respiratory disorders such as chronic obstructive pulmonary disease (COPD) the correlation of quality of life (QOL) and physiological measures of disease severity such as forced expiratory volume in one second $\left(\mathrm{FEV}_{1}\right)$ have been found to be either weak [5-8] or not existent [9], although respiratory symptoms such as wheezing, dyspnea, and subjective severity of breathing problems were more strongly related to QOL $[7,10]$. In a previous study of 64 sarcoidosis patients, we also found that QOL was not associated with pulmonary function tests and SACE [11]. The presence of symptoms, however, influenced QOL considerably [12]. The number and severity of symptoms may therefore provide more accurate information on the degree of impairment and the well-being of patients with sarcoidosis than the routinely performed physiological measures of disease activity and severity.

Other epidemiological studies have shown that there are considerable differences between countries, not only in the prevalence of sarcoidosis but also in the clinical presentation [13]. For the Netherlands, no extensive epidemiological data exist on the clinical presentation of sarcoidosis. Since the symptoms of sarcoidosis vary considerably, it is essential that pulmonary physicians and other physicians such as general practitioners, internists, and rheumatologists are aware of the possible forms of presentation of this disorder.

Therefore, the aim of the present study was to make an extensive inventory of sarcoidosis-related symptoms in order to gain more information on the 
presentation of this disorder. Data on family history, duration of the disease, diagnostic procedures, and treatment were also analyzed.

\section{Materials and methods}

\section{Patients}

All members of the Dutch Sarcoidosis Society $(n=1975)$ were sent the questionnaire by mail together with a letter from the Dutch Sarcoidosis Society asking patient members to participate in a study on the clinical presentation of sarcoidosis. Of the 1975 members, 1755 members were sarcoidosis patients. Of these, 1093 sarcoidosis patients $(62.3 \%)$ responded and $1026(58.5 \%)$ completed the questionnaire. The remaining 67 persons gave a number of reasons why they did not wish to participate in the study; 1) too many questions (14), 2) no time (10), 3) no symptoms (9), and 4) the diagnosis was made too long ago. Eight persons returned the questionnaire without giving a reason for not filling it in.

\section{Questionnaire}

The questionnaire consisted of forty-three items including questions concerning socio-demographic data and medical data such as medical history, family history, duration of disease, diagnostic procedures, treatment, symptoms at onset of the disease and current symptoms. Most of the questions were multiple choice, sometimes giving the possibility to tick more than one answer. A number of questions were open-ended, allowing patients to give information concerning their personal situation. The questionnaire was not standardized. It was pre-tested in a subpopulation of ten sarcoidosis patients.

\section{Statistical analysis}

The occurrence of reported symptoms, diagnostic procedures, treatment, and family history were analyzed by frequencies. Data are expressed as absolute numbers, in 
percentages, and, if appropriate, in mean \pm SD. In order to detect statistically significan differences, Chi-squared tests, Pearson tests, Spearman tests, log-linear analyses ant Student"s $t$-tests were used. Probability values smaller than 0.01 were considered tt be statistically significant. All analyses were performed using the Statistical Packagi for Social Sciences (SPSS).

\section{Results}

Characteristics of the study population are summarized in table I.

Table 1. - Characteristics of the Dutch sarcoidosis patient population

Total number of cases

Gender: male/female

Actual age, years*

Age at the time of diagnosis, years*

Years since diagnosis*

Years since diagnosis: more/less than 2 years

Familiar sarcoidosis yes/no

Symptoms at the time of diagnosis yes/no

Symptoms at the time of the study yes/no

Current smoking yes/no

Corticosteroids yes/no
1026

$380 / 646(37 / 63)$

$46.7 \pm 11.6$

$36.5 \pm 10.9$

$12.2 \pm 10.9$

$945 / 81(92 / 8)$

$170 / 856(16.5 / 83.5)$

$923 / 54(90 / 5)$

938/88 (91/9)

$115 / 875(11 / 86)$

$565 / 367(55 / 36)$

Data are expressed as total numbers with percentages in parentheses. * Data are expressed as mean $\pm \mathrm{SD}$.

Men and women differed significantly with respect to age. Twenty-six percent of the women were older than 45 years at diagnosis, compared with only $11.3 \%$ of the men. Mean age at diagnosis for women was 37.6 years and for men 34.7 years (unequal variance $t$-value $4.2, \mathrm{p}<0.001$ ). Also at the time of the study the women were significantly older than the men (unequal $t$-value $3.1, p<0.01$ ).

With respect to marital status, 715 patients $(69.7 \%)$ were married, $101(9.8 \%)$ were single, $35(3.4 \%)$ were divorced, and $28(2.7 \%)$ were widowed. It appeared 
that more men than women were living together with a partner $\left(\chi^{2}=8.49\right.$, $\mathrm{p}<0.005$ ). With respect to ethnic origin, only $0.5 \%$ of the study population were of African descent, all others were Caucasians.

The time interval between the onset of symptoms and the diagnosis was less than three months in $25.5 \%$ of cases, three to six months in $17.6 \%$ of cases, six to twelve months in $11.6 \%$ of cases, and more than one year in $24.5 \%$ of cases. This information was missing in $20.8 \%$ of cases. A correlation was found between age and time interval between onset of symptoms and diagnosis (Pearson's correlation coefficient $0.15, \mathrm{p}<0.00 \mathrm{I}$ ), older patients were diagnosed later.

In the course of their disease 565 patients $(55.1 \%)$ were treated with systemic corticosteroids, $322(31.4 \%)$ with inhaled corticosteroids, and $323(31.5 \%)$ with nonsteroidal anti-inflammatory drugs. No data were available with respect to doses and/or duration of treatment.

Familial sarcoidosis was reported by 170 patients (16.5\%). In 106 of these cases ( $62 \%$ ) a first-degree relative was affected (in 47 cases brother/sister, in 28 cases the mother, in 20 cases the father, and in 11 cases a child). In 75 cases second-degree relatives suffered from sarcoidosis and in 14 cases two or three relatives were affected by sarcoidosis.

A positive family history with respect to Crohn "s disease was reported by 66 patients $(6.4 \%)$. In 50 of these cases $(75.7 \%)$ first-degree family members were affected. Four patients $(0.4 \%)$ of this study population suffered from both sarcoidosis and Crohn's disease.

Table II summarizes the symptoms reported by the patients at onset of the disease and at the time of the study.

The most frequent symptom at presentation of the sarcoidosis as well as at the time of the study was fatigue. No correlation was found between the symptom fatigue and age. Other frequently reported symptoms were coughing, arthralgia, reduced exercise capacity, muscle pain, and dyspnea. Heart-related problems - including arrhythmias, palpitations, angina pectoris, orthopnea and paroxysmal nocturnal dyspnea -were reported by $9.6 \%$ of patients at the onset 
of the disease and by $9.9 \%$ at the time of the study, and a dry mouth by $15.1 \%$ of patients at the onset of the disease, and $15.7 \%$ at the time of the study. At the time of the study only 171 patients (16.6\%) did not report any dyspnea, while 396 patients $(38.6 \%)$ reported dyspnea at heavy exertion, 314 patients $(30.6 \%)$ experienced dyspnea when performing routine daily activities, and 7 patients $(0.7 \%)$ while at rest. The remaining 138 patients $(13.5 \%)$ did not answer this question.

Women reported more symptoms than men, when age and marital status were entered as covariates $(F=20.5, p<0.001)$. Moreover, results indicated that more women than men had symptoms $\left(x^{2}=5.62, p<0.05\right)$.

Table II. - The most common symptoms related to sarcoidosis at the time of diagnosis as well as at the time of this study $(n=1026)$

\begin{tabular}{lcccc}
\hline Symptom & \multicolumn{2}{c}{ At time of diagnosis } & \multicolumn{2}{c}{ At time of study } \\
& $\mathrm{n}$ & $\%$ & $\mathrm{n}$ & $\%$ \\
\hline Dyspnea & 771 & 75.1 & 717 & 69.9 \\
Cough & 548 & 53.4 & 306 & 29.8 \\
Weight loss & 275 & 26.8 & 106 & 10.3 \\
Thoracic pain & 321 & 37.3 & 280 & 27.3 \\
Fatigue & 791 & 77.1 & 728 & 71.0 \\
Arthralgia & 535 & 52.1 & 536 & 52.2 \\
Skin lesions & 349 & 34.0 & 177 & 17.3 \\
Visual impairment & 323 & 31.5 & 279 & 27.2 \\
Muscle pain & 383 & 37.3 & 402 & 39.2 \\
General weakness & 262 & 25.5 & 230 & 22.4 \\
Starting problems & 330 & 32.2 & 338 & 32.9 \\
Reduced exercise capacity & 498 & 48.5 & 518 & 50.5 \\
Increased need for sleep & 529 & 51.6 & 302 & 29.4 \\
Headache & 278 & 27.1 & 163 & 15.9 \\
Dizziness & 250 & 24.4 & 99 & 9.6 \\
Depressive symptoms & 218 & 21.2 & 169 & 16.5 \\
Tension, strain & 317 & 30.9 & 262 & 25.5 \\
\hline
\end{tabular}


Women suffered more frequently from arthralgia $\left(\chi^{2}=18.95, \mathrm{p}<0.001\right)$, muscle pain $\left(\chi^{2}=7.91, p<0.01\right)$, chest pain $\left(\chi^{2}=16.77, p<0.001\right)$, fatigue $\left(\chi^{2}=5.44, p<0.05\right)$, starting problems $\left(\chi^{2}=12.56, p<0.001\right)$, a dry mouth $\left(\chi^{2}=3.22, p<0.01\right)$, as well as skin lesions $\left(\chi^{2}=5.84, p<0.05\right)$.

At the onset of the disease only $8.7 \%$ of patients suffered from just one symptom, $13.8 \%$ suffered from two symptoms, $16.4 \%$ from three symptoms, and $12.5 \%$ from four symptoms. These percentages did not change significantly over the course of the disease. Correlations were found between the duration of the disease and the symptoms muscle pain $(r=0.14, \mathrm{p}<0.01)$, arthralgia $(r=0.11$, $p<0.01)$, heart problems $(r=0.14, p<0.001)$, skin lesions, e.g., erythema nodosum $(r=0.12, p<0.001)$, visual impairment $(r=0.10, p<0.01)$, dry mouth $(r=0.10$, $\mathrm{p}<0.01)$ and reduced exercise capacity $(\mathrm{r}=0.09, \mathrm{p}<0.01)$, respectively.

Table III summarizes the diagnostic procedures that were performed to establish the diagnosis of sarcoidosis in these patients.

Table III. - Diagnostic procedures involved in the assessment of sarcoidosis

\begin{tabular}{lcc}
\hline Procedure & number & $\%$ \\
\hline Chest radiography & 908 & 88.5 \\
(HR)CT-scan of the thorax & 390 & 38.0 \\
Laboratory tests & 794 & 77.4 \\
Lung function test & 712 & 69.4 \\
Exercise tests & 352 & 34.3 \\
Bronchoscopy & 573 & 55.8 \\
Bronchoalveolar lavage & 248 & 24.2 \\
Mediastinoscopy & 200 & 19.5 \\
Thoracoscopy & 272 & 26.5 \\
Liver biopsy & 82 & 8.0 \\
Renal biopsy & 37 & 3.6 \\
Skin biopsy & 160 & 15.6 \\
Eye check & 284 & 27.7 \\
\hline
\end{tabular}

(HR)CT: (High resolution) computed tomography 
The most frequent tests were chest radiographs in 908 patients $(88.5 \%)$, laboratory tests in 794 patients $(77.4 \%)$, and pulmonary function tests in 712 patients $(69.4 \%){ }^{67} \mathrm{Ga}$ scans were performed in four cases. Various correlations were found between the duration of the disease and the use of diagnostic procedures. In $50 \%$ of the patients who were diagnosed within the previous four years (high resolution) computed tomography ((HR)CT) of the chest was performed, compared with only $30.8 \%$ of those diagnosed more than ten years ago ( $r=0.18, p<0.001)$. Bronchoalveolar lavage $(B A L)$ was performed in $32 \%$ of the patients diagnosed within the previous four years and in $17 \%$ of those who were diagnosed more than ten years ago $(r=-0.15, \mathrm{p}<0.001)$. Of the patients who had suffered from the disease two to eight years, $12.5 \%$ had undergone mediastinoscopy, compared with $26.5 \%$ of those who were diagnosed more than ten years ago $(r=0.12, p<0.001)$. In patients who were diagnosed within the last two years, thoracoscopy was performed in $17.9 \%$, compared with $32.5 \%$ of those diagnosed more than ten years ago $(r=0.11, \mathrm{p}<0.05)$.

Sarcoidosis was the first diagnosis in $57 \%$ of the patients. Others were first diagnosed as suffering from tuberculosis $(4.8 \%)$ and rheumatoid arthritis (5.1\%). In $3.1 \%$ of cases a psychological cause was first considered responsible for the reported symptoms. In the remaining cases $( \pm 30 \%)$ a large range of disorders was first diagnosed, such as bronchus carcinoma, non-Hodgkin lymphoma, diabetes mellitus, hypertension, and pathology of the oesophagus. Of these diagnoses, bronchus carcinoma was the most frequently reported $(2 \%)$, the others consisted of a large number of different, isolated diagnoses.

Seventy-five percent of this study population indicated that they regularly visited a chest physician and/or other physicians such as the internist (24.3\%), ophthalmologist (29.2\%), rheumatologist (9.8\%), dermatologist (10.1\%), neurologist $(5.9 \%)$, cardiologist $(5.7 \%)$, and ear-nose-throat physician ( $5.8 \%)$ on a regular basis, while $34 \%$ of the patients visited the general practitioner regularly. A relationship was found between the duration of the disease and the number of physicians that were visited on a regular basis $(r=0.07, p<0.03)$. 
Treatment with systemic corticosteroids correlated with duration of the disease (unequal $t$-value $-4.44, \mathrm{p}<0.001$ ) and age (equal $t$-value-3.34, $<<0.001$ ). No relationship was found between the reported symptoms and treatment with systemic corticosteroids. No significant differences with respect to symptoms were found between patients, who had and those who had not been treated with systemic corticosteroids in the course of their disease.

\section{Discussion}

In this study, the symptoms, diagnostic procedures, treatment, and course of disease were evaluated in a large number of sarcoidosis patients.

This survey of members of the Dutch Sarcoidosis Society showed that persistent fatigue was found to be the most common symptom. Remarkably, fatigue was reported more frequently than respiratory symptoms or any other symptom, in contrast with the results of a recent study comparing the clinical picture of sarcoidosis in Finland and Japan [14]. In Finland the main presenting symptoms were coughing (33\%) and in Japan eye symptoms (41\%), whereas fatigue was reported in $21 \%$ of patients in Finland and none in Japan [14]. In our recent study of 64 sarcoidosis patients in the Netherlands, $58 \%$ appeared to have actual symptoms. Of this sub-group $80 \%$ reported fatigue at the time of diagnosis and $77 \%$ still suffered from it at the time of the study [12]. In the present study the patients were allowed to report more than one symptom which was present at the onset of the disease and led to medical examination and the correct diagnosis. Moreover, in about half of the patients of the Finish/Japanese study the disease was detected by mass chest radiograph surveys. In the Netherlands no mass chest radiograph surveys are performed, and this may account for the differences between the sarcoidosis population in the Netherlands and these countries.

Sarcoidosis is a disorder that affects the relatively young. The peak incidence 
for men and women occurs between 20 and 40 years [15]. In agreement with this, in our patient group the mean age was 36 years at the onset of the disease. At this age, persistent fatigue and reduced exercise capacity can be expected to interfere severely not only with patients" daily activities but also with their working life.

Although fatigue is a common symptom of many chronic diseases [3], neither the frequency nor the physiological basis of this symptom in sarcoidosis has been studied. Fatigue can be assessed by appropriate QOL instruments such as the World Health Organization Quality of Life assessment instrument (WHOQOL-100) [16], or special fatigue measures such as the Profile of Fatigue-Related Symptoms (PFRS) [17], designed for the chronic fatigue syndrome (CFS). However, data obtained by means of a questionnaire have to be regarded as subjective. If fatigue remains the main symptom, it may be difficult to demonstrate that the patient's impairment is due to sarcoidosis, e.g., for insurance purposes or questions of patients' work capability. Therefore, the physiological basis of fatigue in sarcoidosis needs to be studied and markers must be found in order to assess this symptom more objectively. Recently, we found a relationship between the level of acute phase response proteins and fatigue in sarcoidosis patients [18].

A number of other persistent physical symptoms such as reduced exercise capacity, arthralgia, weakness, starting problems, and muscle pain were reported. The question then arises as to whether these could be expression of muscle involvement. This would be remarkable, considering that symptomatic muscle involvement in sarcoidosis is estimated to occur in only $1.4-2.3 \%$ of cases [19]. Asymptomatic muscle involvement, however, has been reported in $50-80 \%$ of cases [20]. In a previous study we showed that reduced respiratory muscle endurance time was related to impaired mobility in a group of sarcoidosis patients, although, no such relationship was found with peripheral muscle strength [11].

Although in this study the patients reported mainly physical symptoms, 
almost a third of patients also suffered from psychosocial problems such as feeling depressed, tension and strain at the onset of the disease. These problems improved only slightly over time. The latter symptoms were attributed to sarcoidosis by the patients themselves. Previously, we demonstrated that QOL factors were associated with depressive symptoms in sarcoidosis [12]. Thus sarcoidosis patients may benefit from psychosocial support and learning different coping strategies as well as attention to the somatic aspects of the disease [12]. Further intervention research is needed here, however.

In this study population, $46 \%$ had suffered from sarcoidosis for more than eight years. This group reported symptoms such as arthralgia and muscle pain as well as reduced exercise capacity more frequently than patients who were diagnosed less than six years ago. Surprisingly, the same tendency was found for skin lesions such as erythema nodosum. These findings suggest that such persistent symptoms not only impair health status and quality of life of patients with a chronic course of sarcoidosis, but also represent a large demand on health facilities. This demand may mean that the patients make frequent visits to various physicians and require prolonged medication. This may also have economical implications. However, for this patient group no data are available on frequency of visits to the various physicians. Duration of the disease was related only moderately to the number of physicians visited on a regular basis.

Sarcoidosis is thought to occur more frequently in women than in men [21]. The study population of PIETINALHO and OHMICHI consisted of $59 \%$ and $55 \%$ women in Finland and in Japan, respectively [14]. A recent epidemiological study from Spain, where sarcoidosis is a rare disorder, reported $85 \%$ women in their study population [22]. In our study $63 \%$ of the study population were women.

In a study in the USA, familial sarcoidosis was estimated to occur in $5 \%$ of cases among Caucasians and in 19\% among African Americans [23]. In the present patient group the percentage of familial sarcoidosis was much higher $(16.5 \%)$ than expected, since only $0.5 \%$ of patients were of African descent. In 
a study of the members of the German sarcoidosis patient organization, a familial sarcoidosis was found in $7.5 \%$ of cases [24]. In the clinical practice, however, familial sarcoidosis is seen only occasionally and large-scale epidemiological studies are needed to identify "sarcoidosis families" and undertake the necessary genetic research.

Both sarcoidosis and Crohn's disease are disorders of unknown origin and are characterized by granulomatous inflammation. As the histological characteristics of sarcoidosis and Crohn's disease are similar, a pathogenic relationship has been suggested [25]. In one family GrÖNHAGEN-RISKA et al. found an accumulation of both [26]. Cases have also been described, in which patients met the criteria for both, Crohn's disease and sarcoidosis [25]. In our study population only four patients reported suffering from both diseases.

In this present study sarcoidosis was the initial diagnosis in only $57 \%$ of cases, suggesting that the diagnosis of the disease can be difficult. Other first diagnoses included a wide range of somatic disorders. Also psychological problems were considered to be the cause of the symptoms in a number of patients. Furthermore, only about $25 \%$ of the patients were correctly diagnosed within the first three months after the onset of symptoms and in another $25 \%$ it took more than one year. A relationship was found with the age of the patients; older people experienced longer delay.

Among the diagnostic procedures used computed tomography appeared relatively little used (38\%), although especially its high resolution form(HRCT) is much more sensitive than plain radiographs and can demonstrate early fibrosis and distortion of the lung parenchyma [4,27]. Also, bronchoalveolar lavage (BAL) was relatively little used, considering its importance in the diagnosis of sarcoidosis [28]. Lung biopsies obtained by thoracoscopy and mediastinoscopy were performed relatively often, even though these tests are much more invasive. However, when the use of these tests was correlated with the duration of the disease, we found that in patients with a shorter time since diagnosis, (HR)CT and BAL were performed more often and mediastinoscopy 
and thoracoscopy less often than in those diagnosed more than eight years ago. This reflects recent shifts in management strategies resulting from the development of new diagnostic techniques. The use of BAL together with (HR)CT obviates the need for more invasive diagnostic procedures such as thoracoscopy and mediastinoscopy in sarcoidosis.

There are several limitations to the present study. Since the data were collected by means of questionnaires completed only by the patients themselves, medical data from their physicians with respect to severity and stage of the disease are lacking. In this patient population it appears that a large number of tests were performed to obtain histological evidence of granulomatous inflammation.

Corticosteroids are regarded as the most effective treatment options for severe symptoms or involvement of eyes, central nervous system or the heart [21]. For pulmonary sarcoidosis, objective evidence of deterioration in pulmonary functions has been suggested as an indication of the need for supportive treatment [29]. Recently, small long-term advantages from prolonged treatment with systemic corticosteroids were found for patients with pulmonary sarcoidosis and persistent radiographic shadowing [30]. In contrast, two other studies have found that the rate of relapse was higher for patients treated with corticosteroids than for untreated patients $[31,32]$. In the present study, $55.1 \%$ of patients have received systemic corticosteroids, this is within the range of $30 \%$ to $100 \%$ reported in various other studies [13]. In the present study no relationship was found between the number and kind of symptoms and treatment with corticosteroids. Duration of disease and age, however, were found to be related to treatment with systemic corticosteroids. Inhaled corticosteroids had been used at some time in the course of the disease by $31 \%$ of patients, even though these are generally not recommended for the treatment of sarcoidosis [33]. However, bronchial hyperresponsiveness is assumed in about $30 \%$ of patients with sarcoidosis [34], and this may explain the positive effect of inhaled corticosteroids on respiratory symptoms [35]. Moreover, 31\% of patients 
received non steroidal anti-inflammatory drugs (NSAIDs). NSAIDs are sometimes used to treat minimal disease [36], they may not only help to relieve arthralgia and muscle pain but also have an anti-inflammatory effect in sarcoidosis.

In conclusion, the results of this study within a sarcoidosis patient population in the Netherlands indicated that patients present a wide range of physical symptoms, the most common being persistent fatigue. A considerable number of patients suffered from depressive feelings, tension, and strain, many of which persisted over time. No relationship was found between the reported symptoms and treatment with systemic corticosteroids. As the symptom fatigue is difficult to assess objectively, the physiological basis of fatigue in sarcoidosis has to be studied and markers are needed for its accurate assessment. Due to the broad range of possible symptoms, patients with sarcoidosis may consult the family doctor, pulmonologist or any other physician. Therefore, physicians in general should be aware of the many possible presentations of this disorder. Large-scale prospective studies are needed to gain more insight into sarcoidosis, which may be facilitated by a data base, since sarcoidosis remains a relatively rare disorder. In addition, the quality of life and health status should form part of the monitoring of this patient group.

\section{Acknowledgements}

The authors would like to thank the Dutch Sarcoidosis Society for their assistance in collecting the data, the participating members of the organization for their cooperation, and V. Jones for her editorial help. This study was financially supported by a grant from the Dutch Government Department of Health, Welfare and Sports. 


\section{References}

1. Thomas PD, Hunninghake GW. Current concepts of the pathogenesis of sarcoidosis. Am Rev Respir Dis 1987; 135: 747-760.

2. Newman LS, Rose CS, Maier LA. Sarcoidosis. N Engl J Med 1997; 336:12241234.

3. James DG. Complications of sarcoidosis. Chronic fatigue syndrome. Sarcoidosis $1993 ; 10: 1-3$.

4. Costabel U, du Bois RM, Eklund $\mathrm{A}$, et al. Consensus conference: activity of sarcoidosis. Third WASOG meeting, Los Angeles, USA, September 8-11, 1993. Eur Respir J 1994; 7: 624-627.

5. McSweeny AJ, Grant I, Heaton RK, Adams KM, Timms RM. Life quality of patients with chronic obstructive pulmonary disease. Arch Intern Med 1982; 142: 473-478.

6. Jones PW, Quirk FH, Baveystock CM, Littlejohns P. A self-complete measure of health status for chronic airflow limitation. The St. George's Respiratory Questionnaire. Am Rev Respir Dis 1992; 145: 1321-1327.

7. Schrier AC, Dekker FW, Kaptein AA, Dijkman JH. Quality of life in elderly patients with chronic nonspecific lung disease seen in family practice. Chest 1990; 98: 894-899.

8. Renwick DS, Connolly MJ. Impact of obstructive airways disease on quality of life in older adults. Thorax 1996; 51: 520-525.

9. Okubadejo AA, Jones PW, Wedzicha JA. Quality of life in patients with chronic obstructive pulmonary disease and severe hypoxaemia. Thorax 1996; 51:44-47.

10. Maillé AR, Koning CJM, Zwinderman AH, Willems LNA, Dijkman JH, Kaptein A.A. The development of the 'Quality-of-Life for Respiratory Illness Questionnaire' (QOL-RIQ): a disease-specific quality-of-life questionnaire for patients with mild to moderate chronic nonspecific lung disease. Respir Med 1997; 91: 297-309.

11. Wirnsberger RM, Drent M, Hekelaar N, et al. Relationship between respiratory muscle function and quality of life in sarcoidosis. Eur Respir $J 1997 ; 10: 1450-$ 1455.

12. Drent M, Wirnsberger RM, Breteler MHM, Kock LMM, De Vries J, Wouters EFM. Quality of life and depressive symptoms in patients suffering from sarcoidosis. Sarcoidosis Vasc Diffuse Lung Dis 1998; 15: 59-66.

13. James DG. Epidemiology of sarcoidosis. Sarcoidosis 1992; 9: 79-87.

14. Pietinalho A, Ohmichi M, Hiraga $Y$, Löfroos A-B, Selroos O. The mode of presentation of sarcoidosis in Finland and Hokkaido, Japan. A comparative analysis of 571 Finnish and 686 Japanese patients. Sarcoidosis 1996; 13:159-166.

15. Hillerdal G, Nöu E, Osterman K, Schmekel B. Sarcoidosis: epidemiology and prognosis. A 15-year European study. Am Rev Respir Dis 1984; 130: 29-32.

16. WHOQOL group. Field trial WHOQOL-100 February 1995, facet definitions and questions. $1995 \mathrm{a}$; Geneva: WHO (MNH/PSF/95. 1.B).

17. Ray C, Weir WRC, Phillips S, Cullen S. Development of a measure of symptoms in chronic fatigue syndrome: The Profile of Fatigue-Related Symptoms (PFRS). Psychol Health 1992; 7: 27-43. 
18. Drent M, Schols AMWJ, Wouters EFM. Alterations in metabolism and body composition associated with an acute phase response in patients suffering from sarcoidosis. Sarcoidosis Vasc Diffuse Lung Dis 1997; 14(suppl 1): 43.

19. Jamal MM, Cilursu AM, Hoffman EL. Sarcoidosis presenting as acute myositis. Report and review of the literature. $J$ Rheumatol 1988;15:1868-1871.

20. Silverstein A, Siltzbach LE. Muscle involvement in sarcoidosis: asymptomatic, myositis, and myopathy. Arch Neurol 1969;21:235-241.

21. du Bois RM. Sarcoidosis. In: Walters EH, du Bois RM, eds. Immunology and Management of Interstitial Lung Diseases. London: Chapman and Hall, 1995; 97 122.

22. Fité E, Alsina JM, Mañă J, Pujol R, Ruiz J, Morera J. Epidemiology of sarcoidosis in Catalonia: 1979-1989. Sarcoidosis 1996;13:153-158.

23. Harrington DW, Major M, Rybicki B, Popovich J, Maliarik M, Iannuzzi MC. Familial sarcoidosis: analysis of 91 families. Sarcoidosis 1994; 11:240-243.

24. Kirsten D. Sarkoidose in Deutschland. Analyse einer Fragebogenaktion im Jahre 1992 bei Patienten der Deutschen Sarkoidose Vereinigung. Pneumologie 1995; 49: 378-382.

25. Johard U, Berlin M, Eklund A. Sarcoidosis and regional enteritis in two patients. Sarcoidosis 1996; 13: 50-53.

26. Grönhagen-Riska C, Fyhrquist F, Hortling L, Koskimies S. Familial occurrence of sarcoidosis and Crohn's disease. Lancet 1983; 1: 1287-1288.

27. Hansell DM. High resolution computed tomography in sarcoidosis and extrinsic allergic alveolitis: imaging insights. Sarcoidosis 1992; 9: 21-28.

28. Drent M, Van Nierop MAMF, Gerritsen FA, Wouters EFM, Mulder PGH. A computer program using BALF-analysis results as a diagnostic tool in interstitial lung diseases. Am J Respir Crit Care Med 1996; 153: 736-741.

29. Hunninghake GW, Gilbert S, Pueringer R, et al. Outcome of the treatment for sarcoidosis. Am J Respir Crit Care Med 1994; 149: 893-898.

30. Gibson GJ, Prescott RJ, Muers MF, et al. British Thoracic Society Sarcoidosis study: effects of long term corticosteroid treatment. Thorax 1996; 51:238-247.

31. Gottlieb JE, Israel HL, Steiner RM, Triolo J, Patrick H. Outcome in sarcoidosis. The relationship of relapse to corticosteroid therapy. Chest 1997; 111:623-631.

32. Eule H, Weinecke A, Roth I. The possible influence of corticosteroid therapy on the natural course of pulmonary sarcoidosis. Ann NY Acad Sci 1986; 465:695-701.

33. Kirsten D. Inhaled steroids for sarcoidosis? Editorial. Eur Respir J 1995; 8: 679681.

34. Kirsten D, Nowak D, Pielesch W, Magnussen H. Hyperreaktivität und obstruktive Ventilationsstörung bei Lungensarkoidose. Pneumologie 1992; 46: 278-279.

35. Dutch Study Group on Pulmonary Sarcoidosis, Alberts C, Van der Mark ThW, Jansen HM. Inhaled budesonide in pulmonary sarcoidosis: a double-blind, placebo-controlled study. Eur Respir J 1995; 8: 682-688.

36. Baughman RP, Lower EE. Alternatives to corticosteroids in the treatment of sarcoidosis. Sarcoidosis Vasc Diffuse Lung dis 1997; 14: 121-130. 
Chapter 4

Quality of life and depressive symptoms in patients suffering from sarcoidosis 


\section{Quality of life and depressive symptoms in patients suffering from sarcoidosis}

M. Drent*, R.M. Wirnsberger*, M.H.M. Breteler**, L.M.M. Kock**, J. de Vries***, E.F.M. Wouters*

*Department of Pulmonology, University Hospital Maastricht, **Department of Clinical Psychology and Personality, University of Nijmegen, ${ }^{* * *}$ Department of Psychology, Tilburg University, The Netherlands

Sarcoidosis Vasc Diffuse Lung Dis 1998; 15: 59-66 


\section{Abstract}

Apart from the disease status, chronically ill patients are confronted with stressors like dependence, limitations in mobility and physical complaints. Data on patients with sarcoidosis, however, are lacking. The aim of this study was to investigate the quality of life (QOL) and the influence of QOL factors on depressive symptoms in these patients.

Sixty-four patients with histologically proven sarcoidosis participated in this study. Significant co-morbidity was excluded. The Sickness Impact Profile (SIP) was used to determine the QOL. Depressive symptoms were measured with the Beck Depression Inventory (BDI), of which a subset of items measured cognitive symptoms, the Cognitive Depression Index (CDI). Disease status was assessed by pulmonary function parameters $\left(\mathrm{FEV}_{1}, T \mathrm{~L}, \mathrm{CO}\right)$, complaints and illness duration. To control for a confounding cognitive style of self-report, the Positive Affect Negative Affect Schedule (PANAS) was administered.

The major complaint was fatigue. QOL was related to the perception of complaints, but not to the assessed disease status. In a multivariate regression $86 \%$ of the variance could be explained in BDI scores, and $83 \%$ in CDI scores. After controlling for demographical factors, disease status and cognitive style, QOL contributed to the regression, explaining another $17 \%$ of variance of $B D I$ scores as well as $\mathrm{CDI}$ scores. Problems with sleeping were associated positively with depressive symptoms in general $(\beta=0.38)$ and depressive cognitions only ( $\beta=0.32$ ).

In sarcoidosis, QOL factors were associated with depressive symptoms. These results suggest that patients with sarcoidosis may profit from attention to the psychosocial as well as the somatic aspects of this disease. 


\section{Introduction}

Clinical manifestations of sarcoidosis depend on the intensity of the inflammation and organ systems affected. Sarcoidosis presents itself in a variety of ways. About $20 \%$ to $50 \%$ of patients have respiratory symptoms including cough, dyspnea, pain in the chest, wheezing, and chest discomfort [1]. Furthermore, fatigue, arthralgia, and erythema nodosum may be the presenting features of sarcoidosis. Because there is no single test that reflects accurately the progression of sarcoidosis, clinicians use clinical examination, chest roentgenograms, and lung function tests to evaluate and monitor the disease process. Furthermore, to date, the specificity of the markers of disease activity is rather low and their clinical value is limited and uncertain $[1,2]$.

Sarcoidosis occurs throughout the world. The estimated rate of incidence of sarcoidosis variates in Europe from 0.5 per 100,000 inhabitants in Italy to 19.0 per 100,000 in Sweden as well as in the Netherlands. The peak incidence of sarcoidosis occurs between the ages 20 and 40 in both men and women, a time when being ill creates significant distress, especially with concern to psychosocial and economic problems [3]. It is reasonable to suggest that - as well as in other chronic disorders - in sarcoidosis disabilities also interfere with daily activities. Moreover, the quality of life (QOL) of these patients can be decreased because of social isolation and economic disadvantages. To date, the impact on performance of routine daily activities associated with this disease has not been measured. Nowadays QOL questionnaires are used to quantify average changes in health and the effect on the patients' daily life and well-being [4-11]. They reflect, and aggregate together, a range of disease activities and their resulting disability and distress. Unlike pulmonary function parameters, such as the forced expiration volume in one second $\left(\mathrm{FEV}_{1}\right)$ - used in pulmonary disorders - these measures correlate quite well with exercise capacity $[2,12,13]$. One of the most widely used questionnaires in chronic diseases is the sickness impact profile (SIP). The SIP is a generic instrument that is applicable in a wide variety 
of diseases, a feature that allows comparison of results across studies. The SIP was intended to measure the impact of sickness rather than to objectively measure the presence of disease or to subjectively assess illness. It is an evaluation of an individual's own experience of illness perceived through its effect on daily activities, feelings, and attitudes [6]. Using the SIP, SCHrIER et al. [4] found that elderly patients with chronic nonspecific lung disease (CNSLD) experienced substantial problems in the area of physical as well as psychological functioning and reported more dysfunction compared to a healthy control group. Furthermore, these latter authors reported that pulmonary function is hardly related to quality of life assessed by SIP-scores. In contrast, RENWICK and ConNOLLY reported that the impairment of the QOL assessed by the St George's Respiratory Questionnaire, or health related QOL, in adults with obstructive airways disease was related to both baseline pulmonary function and nonspecific bronchial responsiveness [14].

Clinical depression and a depressed mood have often been reported to be associated with a lowered QOL $[13,15-17]$. It is well known that a chronic physical illness might be accompanied by depressive symptoms. The classic symptoms of depression - such as fatigue, and sleep disorders - may mimic those of a chronic illness. Therefore, any attempt to study depression among the chronically physically ill must assess and control for the severity of illness [3, $7,15]$. As others, OKUBADEJo et al. [12] recently reported impairment in most areas of QOL in patients with chronic obstructive pulmonary disease (COPD). In addition, these patients suffer from depression and impairment of cognitive function [13]. KLoNofF and KLeINHENZ [3] evaluated the psychological and psychosocial status of patients with sarcoidosis. These latter authors suggested that the diagnosis of sarcoidosis, in and of itself, is not associated with any specific common psychiatric disorder. However, the clinical picture they present (anxiety, depression, and stress in combination with often vague physical symptoms) may be contributing to the misdiagnosis of their condition as a somatization disorder [3]. Although the cause of sarcoidosis still is 
unknown, there is evidence for a central role of cellular immunity in sarcoidosis. Since it has been suggested that stress affects immunity, stress-reduction therapy might be an important adjunct to medical care of sarcoidosis. Thus, it is important to objectively document the clinical status of patients in order to ascertain any beneficial effect of medical treatment. Moreover, if the underlying medical condition in sarcoidosis cannot be improved appropriately by pharmacological management, one should seek to maximize the functional and psychological condition of the patient.

The present study assesses QOL in patients suffering from sarcoidosis and the association of QOL with depressive symptoms.

\section{Materials and methods}

\section{Subjects}

Patients suffering from sarcoidosis from eight participating Dutch hospitals (Rijnstate Hospital, Arnhem; Academic Hospital Nijmegen and Rehabilitation Centre, Dekkerswald, Nijmegen; District Hospital Middle Twente, Hengelo; St. JansHospital, Weert; Maasland Hospital, Sittard; The Wever Hospital, Heerlen; and University Hospital, Maastricht) were selected. The diagnosis of sarcoidosis was based on consistent clinical features, along with biopsy evidence of noncaseating epitheloid cell granulomas. The clinical symptoms of the respective patients vary from none (sarcoidosis detected on routine chest X-ray film) to more or less severe respiratory and general constitutional symptoms or erythema nodosum and arthralgia (i.e. Löfgren's syndrome). None of the participating subjects had any important medical history, which might influence the quality of life. Moreover, patients with significant co-morbidity were excluded. None of the patients had any anti-depressant pharmacotherapy. In order to be able to evaluate the possible impact of the reported complaints, as well as to evaluate the quality of life in patients without reported complaints, these patients were studied separately. According to a difference in 
experiencing physical symptoms the patients were divided into two groups; group I consisted of patients with complaints ( $n=37$ ), whereas group II consisted of those without complaints ( $n=27$ ). Clinical characteristics of the patients are given in table $I$.

In order to be able to evaluate the severity of dysfunction of the sarcoidosis patients a random control group was used as reference value to compare the SIP measurements, which consisted of 594 cases ( 267 males and 327 females; mean age 43 yrs, range 18 75) obtained from a Dutch open population [18].

Table I. - Characteristics of the studied sarcoidosis patient population with separate data for patients with (group I) and without (group II) current complaints

All Group II Group II

Demographic characteristics

Number of cases

Gender (Male/Female)

Age*

Smoking (yes/no)

Medical characteristics

Time from diagnosis years*

$\mathrm{FEV}_{\text {, (normal/decreased) }}$

$T L$, co (normal/decreased)

SACE U/L $(9-25 \mathrm{U} / \mathrm{L})^{*}$

Use of corticosteroids (yes/no)

Psychologicalcharacteristics

Negative Affect"

Positive Affect"

BDI depression*

CDI depression *

PDI depression"

$\begin{array}{lll}64 & 37 & 27 \\ 37 / 27 & 21 / 16 & 16 / 11 \\ 42.7 \pm 11.9 & 45.4 \pm 12.8 & 39.0 \pm 9.6 \\ 11 / 53 & 8 / 29 & 3 / 24\end{array}$

$5.0 \pm 5.5$

$4.4 \pm 5.1$

$6.1 \pm 5.9$

$52 / 12$

$29 / 8$

$23 / 4$

$46 / 18$

$22 / 15$

$24 / 3^{\text {fit }}$

$25 \pm 19$

$30 \pm 25$

$20 \pm 9$

$21 / 43$

$14 / 23$

$7 / 20$

Data are expressed as mean $\pm \mathrm{SD} . \mathrm{FEV}_{1}$ : forced expiration volume in one second; $T \mathrm{~L}, \mathrm{CO}$ : transfer factor of the lung for carbon monoxide; $\mathrm{sACE}$ : serum angiotensin converting enzyme; BDI: Beck Depression Inventory; CDI: Cognitive Depression Index; PDI: Physical Depression Index; $\mathrm{p}=0.05$; " $\mathrm{p}<0.01$ Group I vs. Group II; Student $t$-test. 


\section{Chapter 4}

\section{Procedure}

Seventy-one sarcoidosis patients from eight participating hospitals were contacted.

Seven patients declined, but 64 patients agreed to participate after giving their informed consent. These sarcoidosis patients were studied. The questionnaires were completed under supervision of a research assistant to prevent missing data or misunderstanding. Moreover, the patients were asked about their smoking history and whether they had any of the following complaints, i.e. fatigue, dyspnea, cough, arthralgia or erythema nodosum. The values of the assessed pulmonary function tests and $S A C E$ levels were obtained from the patient record, from the visit closest to the session the questionnaires were completed.

The random control subjects $(n=594)$ were recruited as follows: from a health insurance company, which had 15,942 clients, a random stratified sample of 875 persons were sent the SIP by mail. Five hundred and ninety-four persons (68\%) returned the completed questionnaire.

\section{Assessment procedures}

Lung function parameters which were measured included forced expiration volume in one second $\left(\mathrm{FEV}_{1}\right)$, inspiratory vital capacity (IVC) measured with a pneumotachograph and total lung capacity (TLC) measured with a body plethysmograph (Compactbody, Jaeger, Würzburg, Germany). Measures were selected from the three best efforts. All volumes were expressed as a percentage of the reference value [19]. The transfer factor of the lung for carbon monoxide $(T, C O)$ was measured using the single breath method (Masterlab, Jaeger, Würzburg, Germany).

\section{Modifications in FEV, and $T, \mathrm{CO}$}

In order to compare the FEV, level with the quality of life scores four ranges in the $\mathrm{FEV}_{1}$ as well as $T$, co levels were classified: 1) normal range ( $\left.\geq 80 \%\right)$; 2 ) mild decrease $(60-80 \%) ; 3)$ moderate decrease $(40-60 \%)$ and 4$)$ severe decrease $(<40 \%)$, respectively (according to the American Medical Association classes). 


\section{Questionnaires}

\section{Sickness Impact Profile (SIP)}

QOL was assessed with the SIP (Dutch version) $[4,18]$. The SIP is designed to assess sickness-related behavioral dysfunction. It provides summary scores for physical, psychosocial, and overall behavioral dysfunction, as well as separate scores for 12 categornes of activities. The scores are expressed as the percentages of the maxinal possible score of dysfunction in that particular category or set of categories [5].

\section{Beck Depression Inventory ( $B D I$ )}

Depressive symptoms were measured with the BDI [20]. The BDI is a well validated index of depression and correlates well with diagnostic criteria. The advantage of the $\mathrm{BDI}$ is that it places the subject within a range of depression (none, mild, moderate, and severe), rather than merely identifying if the person meets diagnostic criteria. This instrument consists of statements which are grouped in 21 groups of four possible responses. The patient was asked to select one statement from each group that describes best the way he has been feeling the past week. Each answer is scored on a four-point Likert-type scale of zero to three. A summation of the ratings in the 21 groups indicates the severity of depression. A patient with a total score of 15 or above was considered to have significant depressive symptoms. In previous studies internal consistency mostly was higher than 0.85. BOSSCHER et al. [21] showed in a non-clinical student population the mean score to be $4.5 \pm 5.0$. In order to control for the physical effects of the illness on mood, items referring to problems with fatigue, sleep, sexual dysfunction, eating, weight, and general health were separated into a Physical Depression Index (PDI). The scale based on the 15 remaining items was called the Cognitive Depression Index (CDI). With medical patients, the CDI might be a better predictor of depression because of a reduction in confusion caused by symptoms of physical illness that are similar to symptoms of depression. PETERson et al. [15] demonstrated this to be a valid way of preventing spurious relationships in a patient population with end-stage renal disease. Internal consistency of the CDI in this sample 
is high, $\alpha=0.85$. The 15 items, as with all BDI items, are answered on a four-point Likert-type scale that represents 0 as the absence of a problem, and 3 as an extreme problem, with a total score range of 0 to 45 . For example, for sad feeling the range is 0 (I do not feel sad) to 3 (I am so unhappy that I cannot stand it). Examples of other cognitive symptoms surveyed are guilt, disappointment, failure, and decision-making.

\section{Positive Affect Negative Affect Schedule (PANAS)}

In order to control for the psychological trait of focusing on negative mood or distress, the PANAS was administered [22]. This questionnaire consists of 20 words referring to mood states, 10 positive, 10 negative. On a five-point Likert-type scale respondents can indicate how often he or she feels that way in general. Internal consistency and validity have been found to be higher than 0.80 and intercorrelations between the positive and negative effect scales in general were low, around $r=-0.20$.

\section{Statistical analysis}

The significance of differences concerning personal characteristics was tested using Chi-squared tests for categorical data and Student's $t$ tests for continuous data. To analyze the association of QOL with depressive symptoms multiple regression analysis was performed using $\mathrm{BDI}$ and $\mathrm{CDI}$ scores as the dependent variable. Demographic, medical and psychological factors were entered blockwise before entering QOL. All analyses were performed using the Statistical Package for Social Sciences (SPSS).

\section{Results}

Table I provides a survey of the demographical, medical and psychological characteristics of the studied patients suffering from sarcoidosis. Age and gender were not related to the demographical and medical variables. Figure 1 shows the reported complaints of the sarcoidosis patients with complaints 
(group I; $\mathrm{n}=37$ ) at the time of onset as well as at the moment this study was performed. The major complaint proved to be fatigue. Moreover, those without complaints at the time they filled in the questionnaires reported less complaints at the time of the presentation of sarcoidosis, as well. Only $2 \mathbb{1}$ patients ( 14 with complaints, group I; 7 without complaints, group II) out of the total sarcoidosis population studied were using oral corticosteroids (mean dose $5.7 \pm 2 \mathrm{mg}^{\text {day }}{ }^{-1}$, range $2.5-10 \mathrm{mg}$ ) at the time they filled in the questionnaires.

The 43 other patients had not used corticosteroids for at least three months prior to this study (table 1 ). This rather low dose of corticosteroids did not seem to affect the pulmonary function test results, the experience of complaints or any of the questionnaire scores.

Fifteen out of the 37 patients with complaints (group I; 40.5\%) showed a decreased $T \mathrm{~L}, \mathrm{CO}$, of which 12 demonstrated a mildly decreased $T \mathrm{~L}, \mathrm{CO}$ and three a moderately decreased $T \mathrm{~L}, \mathrm{CO}$, respectively (according to the American Medical Association classes).

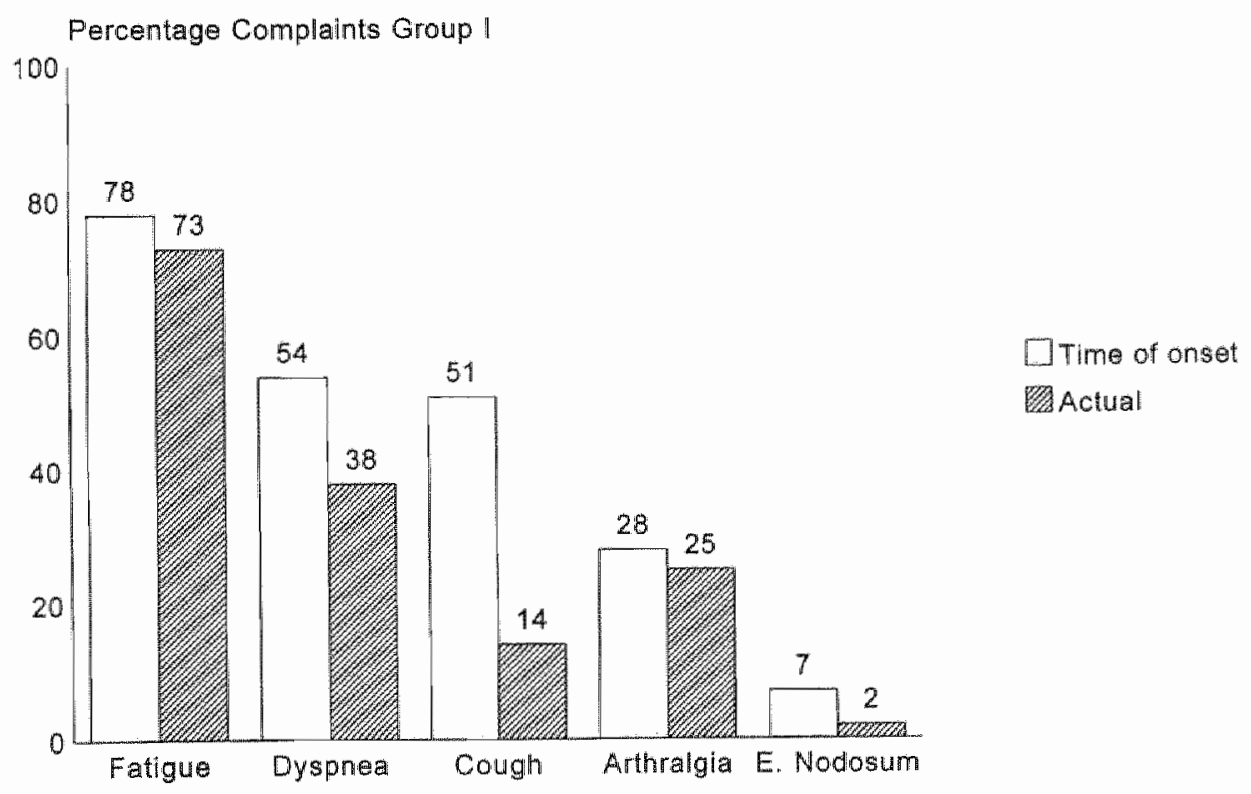

Fig. 1. - Percentages of complaints of the sarcoidosis patients with complaints (group I; $n=37$ ) at the time of onset and the moment the questionnaires were filled in, respectively. E. nodosum: erythema nodosum. 
Only three out of the 27 patients without complaints (group II) showed a mildly decreased $T_{\mathrm{L}}, \mathrm{CO}$, whereas all others showed normal $T \mathrm{~L}, \mathrm{co}$ values. Remarkably, all current smokers of this sarcoidosis patient population showed decreased $T_{L}$, co values. Moreover, those with normal $T_{L}$, co values on average were diagnosed with sarcoidosis three and a half years earlier than those with slightly decreased $T_{L}$, co values (unequal variances, $t$-value $-3.2 d f=54.41$, $\mathrm{p}<0.005)$. Those with decreased $T_{\mathrm{L}}$, co experienced more complaints than those with normal $T \mathrm{~L}$, co values $\left(\chi^{2}=6,6(1), \mathrm{p}=0.01\right)$. Eight out of the 37 patients with complaints (group I; $21.6 \%$ ) showed a mildly decreased $\mathrm{FEV}_{1}$, whereas four out of the 27 patients without complaints (group II; 14.8\%) demonstrated a mildly decreased FEV, (according to the American Medical Association classes). Out of the non-smokers $86 \%$ demonstrated normal $\mathrm{FEV}_{1}$ values, whereas $50 \%$ out of the current smoking sarcoidosis patients showed a mildly decreased FEV, $\left(\chi^{2}=7.2(1), \mathrm{p}<0.005\right)$.

Between the groups of patients with complaints (group D) and without complaints (group II) no difference was found in negative affect, whereas patients without complaints experienced more positive affect (table 1).

Respondents with complaints had significantly higher BDI scores (9.8 \pm 7.8 ) than those without complaints $(4.3 \pm 5.6$; unequal variances $t$ value -3.27 , $d f=61.79, \mathrm{p}<0.005)$. Out of the 64 patients $12(18 \%)$ had a BDI score of 15 or above (associated with significant depression, e.g. Light et al. [13]). Of those 12 cases only one belonged to the group of patients without actual complaints (group II). The average score on the CDI was 3.9t4.6. Univariate analysis still showed those with complaints to have higher CDI scores $(5.2 \pm 5.3)$ than those without $(2.2 \pm 3.6 ; \mathrm{p}<0.01)$.

The results of the SIP subscale scores are given in table II, separately. High percentile scores were found for the patients suffering from sarcoidosis in the subscales employment, recreation and pastimes, and sleep and rest compared to the control group.

Moreover, respondents with complaints had significantly higher percentile 
scores in the SIP subscales mobility (unequal variance $t$-value -3.58 , df 42.4, $\mathrm{p}=0.001$ ), body care and movement (unequal variance $t$-value -2.55 , df 53.2 , $\mathrm{p}=0.01$ ), alertness (unequal variance $t$-value $-3.25, d f 47.3, \mathrm{p}=0.002$ ) as well as emotional behaviour (unequal variance $t$-value $-2.83, d f 54.7, \mathrm{p}=0.007$ ), eating (unequal variance $t$-value $-2.37, d f 47.3, \mathrm{p}=0.02$ ) and home management $(t$ value -2.69, df $62.0, \mathrm{p}=0.01$ ) as compared to those without complaints. Like patients with complaints those without complaints showed higher scores on the subscale sleep and rest as compared to the control group (table II).

Table II. - Average Sickness Impact Profile (SIP) scores of the studied sarcoidosis patient population with separate data for patients with (group I) and without (group II) current complaints and the control group

\begin{tabular}{lllll}
\hline SIP & All & Group I & Group II & $\begin{array}{l}\text { Control } \\
\text { Group }\end{array}$ \\
\hline Alertness Behaviour & $9.4 \pm 17.7^{*}$ & $14.4 \pm 21.0^{*}$ & $2.3 \pm 7.2$ & $4.6 \pm 12.4$ \\
Ambulation & $5.0 \pm 8.2^{*}$ & $5.9 \pm 8.6^{*}$ & $3.6 \pm 7.5$ & $3.0 \pm 7.8$ \\
Body Care and Movement & $3.0 \pm 6.1$ & $4.4 \pm 7.2^{*}$ & $1.0 \pm 3.3$ & $1.9 \pm 5.0$ \\
Communication & $2.9 \pm 4.9$ & $4.4 \pm 6.0$ & $1.6 \pm 3.9$ & $1.1 \pm 4.5$ \\
Eating & $1.5 \pm 3.5$ & $2.3 \pm 4.3$ & $0.4 \pm 1.5$ & $1.0 \pm 3.2$ \\
Emotional Behaviour & $8.5 \pm 13.9$ & $12.1 \pm 16.1^{*}$ & $3.5 \pm 7.7$ & $4.0 \pm 10.0$ \\
Home Management & $8.5 \pm 11.0^{*}$ & $11.5 \pm 11.4^{*}$ & $4.4 \pm 9.0$ & $4.8 \pm 11.8$ \\
Mobility & $4.3 \pm 8.2^{*}$ & $6.8 \pm 9.8^{*}$ & $0.7 \pm 2.5$ & $2.4 \pm 7.6$ \\
Recreation and Pastimes & $14.0 \pm 16.8^{*}$ & $17.5 \pm 18.1^{*}$ & $9.3 \pm 13.9$ & $7.6 \pm 14.1$ \\
Sleep and Rest & $12.9 \pm 16.3^{*}$ & $15.7 \pm 17.6^{*}$ & $9.0 \pm 13.8 *$ & $4.8 \pm 8.2$ \\
Social Interaction & $10.4 \pm 18.1^{*}$ & $12.6 \pm 19.6^{*}$ & $7.3 \pm 15.6$ & $4.0 \pm 8.4$ \\
Work & $14.8 \pm 20.2^{*}$ & $18.0 \pm 20.7^{*}$ & $10.4 \pm 19.1$ & $7.3 \pm 20.0$ \\
Physical dimension & $3.8 \pm 6.1^{*}$ & $5.2 \pm 7.0^{*}$ & $1.6 \pm 3.6$ & $2.2 \pm 5.4$ \\
Psychosocial dimension & $9.9 \pm 14.9^{*}$ & $13.2 \pm 17.0^{*}$ & $5.1 \pm 9.6$ & $3.5 \pm 7.2$ \\
Total SIP score & $8.0 \pm 9.4^{*}$ & $10.4 \pm 10.0^{*}$ & $4.3 \pm 7.1$ & $3.4 \pm 5.6$ \\
\hline
\end{tabular}

Data are expressed as mean $\pm \mathrm{SD}$. Student's $t$-tests: $p<0.01 \mathrm{ws}$. control group. " received from $J_{\text {ACOBS }}$ et al ${ }^{18}{ }^{*}{ }^{*} \mathrm{p} \leq 0.01 \mathrm{vs}$. control group. 


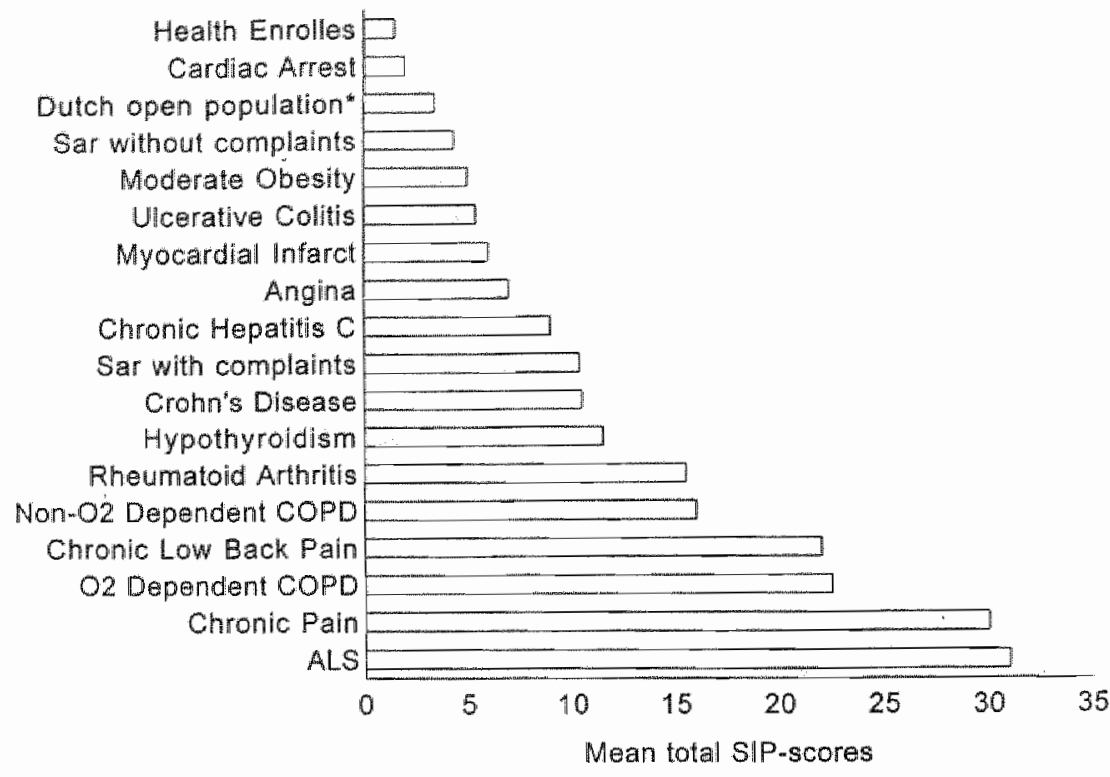

Fig. 2. - Overall Sickness Impact Profile (SIP) scores for different disease conditions or population groups including sarcoidosis patients (Sar) with or without complaints. ALS: amyotrophic lateral sclerosis. (adapted from PATRICK and DEvo [5]; *control group adapted from J ACons et al. [18])

Women showed more emotional $(\mathrm{p}=0.02)$ and body care and movement problems ( $\mathrm{p}=0.04)$. Age was positively related to higher percentile scores in the subscales ambulation $(\mathrm{p}=0.03)$ and mobility $(\mathrm{p}=0.04)$. The duration of the disease, the FEV, and SACE level demonstrated no relation with percentile scores of any of the SIP subscale scores. Patients with decreased $T L$, co values had higher scores on the subscales mobility and eating than those with normal Th, co values.

Figure 2 shows the overall SIP scores for sarcoidosis patients with (group I; $10.4 \pm 10.0$ ) and without complaints (group $I 1 ; 4.3 \pm 7.1$ ), the control population (3.4 5.6) [18] as well as for different disease conditions (adapted from PATRICK and DeYo [5]).

To further analyze the association ofQOL with depressive symptoms multiple regression analysis was performed using $\mathrm{BDI}$ and $\mathrm{CDI}$ scores as the dependent variable. The demographical, medical and psychological variables were forced 
blockwise into the equation, in that particular order. Within each block, the variables were entered stepwise. After having controlled for these variables the functional status variables were entered into the equation. Table III shows the contribution of each block, in terms of explained variance ( $r$ square), and within each block the individual contribution of each variable ( $\beta$ weights). After controlling for demographical, medical, and psychological variables, health status still explained $17 \%$ of the variance in depressive symptoms. Sleeping problems appear to be associated most with both BDI and CDI scores, those with sleeping problems being more depressed. One item of the SIP score, i.e. communication, was not included in the multiple regression analysis because of too many missing values. SIP scores correlated substantially with BDI scores, ranging from $r=0.46$ for body care and movement to $r=0.63$ for sleeping problems. The CDI score still correlated with $r$ mostly above 0.50 (table III).

\section{Discussion}

As far as we know, this is the first study concerning the QOL in sarcoidosis. Our results reveal that sarcoidosis patients are limited in physical and psychological functioning.

They appeared to be particularly affected in the fields of sleep and rest, recreation and pastime, employment, alertness as well as emotional behaviour and social interaction as compared to a control group [18]. This diversity illustrates the broad impact of sarcoidosis on patients' QOL. Compared to patients with CNSLD the fields social interaction and recreation and pastime were more affected in sarcoidosis, whereas ambulation was more affected in CNSLD [4]. Although caution in the interpretation of the comparison is warranted, as most of the scores shown in figure 2 are not standardized for age or sex, the overall SIP scores for sarcoidosis patients with complaints seem comparable with those of patients with Crohn's disease [5]. 
Table III. - Multiple regression of Beck Depression Inventory (BDI) and Cognitive Depression Index (CDI) scores on demographic, medical, and psychological variables, and functional status $(n=54)$

\begin{tabular}{lrr}
\hline & \multicolumn{2}{c}{$\beta^{+}$} \\
Variable & CDI & BDI \\
\hline Block 1 & & \\
$\quad$ Gender & -0.05 & 0.02 \\
Age & -0.06 & 0.01 \\
Smoking & 0.09 & 0.03 \\
& & \\
Block 2 & & \\
Complaints & -0.01 & 0.06 \\
FEV & 0.03 & 0.03 \\
TL,Co & 0.05 & 0.15 \\
Years from diagnosis & 0.10 & 0.10
\end{tabular}

Block 3

Positive Affect

Negative Affect

$\begin{array}{ll}-0.10 & -0.06\end{array}$

$\begin{array}{ll}0.18 & 0.38\end{array}$

Block 4
0.17
0.18

$\mathrm{R}^{2}$

CDI BDI

$\begin{array}{lrr}\text { Alertness Behaviour } & 0.11 & -0.02 \\ \text { Emotional Behaviour } & 0.38 & 0.16 \\ \text { Eating } & -0.07 & 0.03 \\ \text { Home Management } & -0.02 & -0.02 \\ \text { Social Interaction } & -0.14 & 0.04 \\ \text { Body Care and Movement } & 0.01 & 0.04 \\ \text { Ambulation } & 0.12 & 0.09 \\ \text { Mobility } & 0.07 & 0.03 \\ \text { Recreation and Pastimes } & 0.17 & 0.08 \\ \text { Sleep and Rest } & 0.32 & \mathbf{0 . 3 7} \\ \text { Employment } & 0.05 & 0.05\end{array}$

$0.83^{\mathrm{a}} \quad 0.86^{\mathrm{b}}$

$+:$ in final regression equation. Digits printed bold are significant at $\alpha=0.05$. $a: F=7.8$, (20.31) $p<0.001, b: F=9.2(20.31) p<0.001 ; F E V_{j}$ : forced expiration volume in one second; $T L_{,}, \mathrm{C}$ : transfer factor of the lung for carbon monoxide. 
Both sarcoidosis and Crohn's disease are characterized by granuloma formation of the affected tissue. It even has been suggested that the two diseases might be pathogenetically related. Overall SIP scores for sarcoidosis patients without current complaints, general populations, and those who have survived myocardial infarction or cardiac arrest are at the low end of the continuum [5, 18]. Scores for patients with chronic, debilitating illnesses - like COPD - are considerably higher [5].

The univariate statistics showed that patients with complaints experience more depressive symptoms than those without. KLONOFF and KLEnHENZ [3] found a strong association between depressive symptoms and agoraphobic cognitions, suggesting a fear of possible embarrassing situations that may arise due to the complaints. In the multiple regression of the present study the contribution of complaints to depressive symptoms is negligible, however. Note that the impact of a prior existing depression can not be ruled out. Future studies should therefore gather information on a history of depression and low mood, to clear up this issue.

QOL factors, even after control for demographical, medical and psychological factors, were found to be associated with depression in patients with sarcoidosis. Also after exclusion of physical depressive symptoms that may be attributed to the ongoing illness, this relationship remains intact. Moreover, to control for a confounding cognitive style of self-report, the Positive Affect Negative Affect Schedule (PANAS) was administered, a reliable, valid, and efficient means for measuring these two important dimensions of mood [22]. High trait negative affect individuals are more likely to experience intense states of negative affect including anger, contempt, disgust, guilt, fear, and nervousness. The salient finding of the present study is that the SIP-scores, rather than the objective severity of sarcoidosis best predicts the level of cognitive depression, even after controlling for negative affect. Moreover, in chronic lung diseases it has been demonstrated that the relationship between the somatic condition of the patients, reflected by pulmonary function tests, and their quality of life is negligible or weak $[5,14,23]$. In line with this, GuYATT et al. [9] reported that the relationships 
between changes in symptomatic and functional state or QOL in patients with chronic lung diseases and impairment in conventional physical indices are often weak. They found this to be particularly true for interventions such as rehabilitation programs, in which patients are taught to cope with their physiological limitations. Direct measurement of the impact of the illness on patients' lives, therefore, seems necessary to assess whether intervention strategies in this group of patients are of benefit. Thus, future studies to identify valuable variables to measure disease activity will be necessary, since neither the FEV, nor the SACE level in patients suffering from sarcoidosis accounted for a significant variation in the QOL or in depressive cognitions. Only the $T L$, co was demonstrated to be associated with either having complaints or not. In a subpopulation of the present population studied we found the radiographic stage to be related to the SIP subscales "alertness behaviour", "emotional behaviour", "home management' and 'social interaction'. Moreover, respiratory muscle strength and endurance were reduced in these patients with sarcoidosis. However, no relationship between radiographic stage, sACE, respiratory muscle strength, and endurance were found [23].

The chronic inflammatory disorder sarcoidosis interferes with the immune system. It might reduce energy and vitality, and produce progressively worsening, though fluctuating, symptoms that tend to cause psychosocial complaints, and affect the QOL. For this reason, it seems necessary that interventions aimed at improving patients" QOL and reduction of depression should focus on psychological aspects as well as on the chronic and disabling illness. In line with this, as others did [24-27], Speckens et al. [28] suggested cognitive behavioural therapy to be a feasible and effective treatment in general medical patients with more or less unexplained functional somatic symptoms. Basic principles of cognitive behavioural therapy, such as the recognition of the patients' attributions of their symptoms and effective reassurance, could help a large proportion of patients with unexplained symptoms. Sarcoidosis patients, especially those with symptoms not related to measurable functional 
impairment, might benefit from such attention. This might be particularly relevant regarding the high percentage of patients that may suffer from clinical depression. Future studies assessing new intervention strategies are needed to evaluate the benefit of these suggestions.

There are several limitations to the present study. To start with, the crosssectional design prevents causal explanations. The distinct association of complaints with QOL calls for a longitudinal design, which can provide insight into the course of complaints in sarcoidosis, and their influence on QOL. Second, the ratio variables to subjects is high. Future studies should incorporate more subjects, also allowing for more detailed analyses. With regard to the interpretation of the results, caution is required. Both SIP scores and the reported complaints point to the importance of sleeping problems. Although the results of the regression analysis are in line with this conclusion, it should be kept in mind that SIP scores are highly interdependent. Nevertheless, QOL clearly plays a role in depressive symptoms.

In conclusion, these results indicate that in sarcoidosis the quality of life was affected. Extended sleep, severe fatigue, as well as emotional problems seem to be the major problems. Moreover, quality of life factors were associated with depressive symptoms even after controlling for demographical, medical and psychological factors. As far as medical variables are concerned, only the $\pi, c 0$ appears to be slightly related to factors related to quality of life. The diagnostic work-up should explore new approaches in order to elucidate the pathophysiological mechanisms of fatigue in relation to disease activity, such as metabolic aspects, since the regular methods are not sufficient in documenting fatigue. This study points out that interventions aimed at improving patients' quality of life and reduction of depression should focus not only on the disabling illness and somatic care but also on psychological aspects, including coping with the having of sarcoidosis and stress reduction. 


\section{Acknowledgements}

The authors gratefully acknowledge the participating Dutch hospitals, Dr. H.M. Jacobs from the Department of General Practice/Family Medicine, University of Utrecht, for providing the Dutch SIP version and the SIP control data, Dr. A.M.W.J. Schols for the statistical advice, Sylvia Drent and Marjon Elfferich for their great help in collecting the data, as well as their advice and critical comments during this study.

\section{References}

1. Thomas PD, Hunninghake GW. Current concepts of the pathogenesis of sarcoidosis. Am Rev Respir Dis 1987;135: 747-760.

2. Sharma OP, Alam S. Diagnosis, pathogenesis and treatment of sarcoidosis. Curr Opin Pulm Med 1995;1: 392-400.

3. Klonoff EA, Kleinhenz ME. Psychological factors in sarcoidosis: the relationship between life stress and pulmonary function. Sarcoidosis 1993: 118-124.

4. Schrier AC, Dekker FW, Kaptein AA, Dijkman JH. Quality of life in elderly patients with chronic nonspecific lung disease seen in family practice. Chest 1990; 98: 894-899.

5. Patrick DL, Deyo RA. Generic and disease-specific measures in assessing health status and quality of life. Med Care 1989;27: S217-S232.

6. Davies GL, Balart LA, Schiff ER, et al. Assessing health-related quality of life in chronic hepatitis C using the sickness impact profile. Clin Ther 1994; 16: 334343.

7. Sacks CR, Peterson RA, Kimmel PL. Perception of illness and depression in chronic renal disease. Am J Kidney Dis 1990; 15: 31-39.

8. Juniper EF, Guyatt GH, Ferrie PJ, Griffith LE. Measuring quality of life in asthma. Am Rev Respir Dis 1993; 147: 832-838.

9. Guyatt GH, Berman LB, Townsend M, Pugsley SO, Chambers $\mathbb{L}$ W. A measure of quality of life for clinical trials in chronic lung disease. Thorax 1987; 42:773-778.

10. Guyatt GH, Feeny DH, Patrick DL. Measuring Health-related Quality of Life. Ann Intern Med 1993; 118:622-629.

11. Bergner M, Bobitt RA, Carter WB, Gilson BS. The sickness impact profile: development and final revision of a health status measure. Med Care 1981; 19: 787-805.

12. Okubadejo AA, Jones PW, Wedzicha IA. Quality of life in patients with chronic obstructive pulmonary disease and severe hypoxaemia. Thorax 1996; 51:44-47.

13. Light RW, Merrill EJ, Despars JA, Gordon GH, Mutalipassi LR. Prevalence of depression and anxiety in patients with COPD. Relationship to functional capacity. Chest $1985 ; 87: 35-38$. 
14. Renwick DS, Connolly MJ. Impact of obstructive airways disease on quality of life in older adults. Thorax 1996; 51: 520-525.

15. Peterson RA, Kimmel PL, Sacks CR, Mesquita ML, Simmens SI, Reiss D. Depression, perception of illness and mortality in patients with end-stage renal disease. Int J Psychiatry Med 1991; 21: 343-354.

16. McSweeney AJ, Grant I, Heaton RK, Adams KM, Timms RM. Life quality of patients with chronic obstructive pulmonary disease. Arch Intern Med 1982; 142: $473-478$.

17. Mackinnon JR, Avison WR, McCain GA. Pain and functional limitations in individuals with rheumatic arthritis. Ini J Rehabil Res 1994; 17:49-59.

18. Jacobs HM, Luttik A, Touw-Otten FWMM, De Melker RA. The sickness impact profile; results of a validation study of the Dutch version. Ned Tijdschr Geneeskd $1990 ; 134: 1950-1954$.

19. Quanjer PH. Standardized lung function testing. Bull Eur Physiopatol Respir $1983 ; 19: 7-44$.

20. Beck AT, Ward $\mathrm{CH}$, Mendelson M, Mock J, Erbaugh J. An inventory for measuring depression. Arch Gen Psychiat 1961; 4: 561-571.

21. Bosscher RJ, Koning H, Van Meurs R. Reliability and validity of the Beck Depression Inventory in a Dutch college population. Psychol Reports 1986; 58: 696-698.

22. Watson D, Clark LA, Tellegen A. Development and validation of brief measures of positive and negative affect: the PANAS scales.J Pers Soc Psychol 1988; 54 : $1063-1070$.

23. Wirnsberger RM, Drent $M$, Hekelaar $N$, et al. Relationship between respiratory muscle function and quality of life in sarcoidosis. Eur Respir $J 1997 ; 10: 1450-$ 1455.

24. Ketelaars CAJ, Schlösser MAG, Mostert R, Huyer Abu-Saad H, Halfens RJG, Wouters EFM. Determinations of quality of life in COPD patients. Implications for home care. Thorax 1996; 51:39-43.

25. Dudley DL, Glaser EM, Jorgenson BN, Logan DL. Psychosocial conconitants to rehabilitation in chronic obstructive pulmonary disease. Part 1. Psychosocial and psychological considerations. Chest 1980; 77: 413-420.

26. Watson D, Pennebaker JW. Health complaints, stress and distress: exploring the central role of negative affectivity. Psychol Rev 1987; 96:234-254.

27. Wijkstra PJ, Ten Vergert EM, Van Altena R, et al. Long term benefits of rehabilitation at home on quality of life and exercise tolerance in patients with chronic obstructive pulmonary disease. Thorat 1995; 50: 824-828.

28. Speckens AEM, Van Hemert AM, Spinhoven P, Hawton KE, Blok JH, Rooijmans GM. Cognitive behavioural therapy for medically unexplained physical symptoms: a randomised controlled trial. Br Med $J 1995 ; 311: 1328-1332$. 
Chapter 5

Evaluation of quality of life in sarcoidosis patients 


\section{Evaluation of quality of life in sarcoidosis patients}

R.M. Wirnsberger*, J. de Vries**, M.H.M. Breteler***, G.L. van Heck**, E.F.M. Wouters*, M. Drent*

* Department of Pulmonology, University Hospital Maastricht, **Department of Psychology, Tilburg University, *** Department of Psychology and Personality, Nijmegen University, The Netherlands

Respir Med 1998: 92:750-756 


\section{Abstract}

Health-related quality of life (QOL) has become an important topic in health care. However, hardly any attention has been paid to QOL in sarcoidosis. Therefore, the aim of this study was to assess the impact of sarcoidosis on QOL. Sixty-four sarcoidosis patients completed the World Health Organization Quality of Life assessment instrument (WHOQOL-100) and the Beck Depression Inventory (BDI). For the WHOQOL-100 a matched group of healthy controls was selected. Patients were divided into two groups: group I $(\mathrm{n}=37)$ consisted of patients with actual symptoms, group II $(\mathrm{n}=27)$ consisted of asymptomatic patients. The WHOQOL-100 revealed a number of areas in which sarcoidosis patients, especially those with current symptoms, experienced problems. A major symptom in both groups of sarcoidosis patients was fatigue. No association between the facet fatigue and the domain psychological health was found. Depressive symptoms (BDI) were associated with psychological function (WHOQOL-100). No association between pulmonary function tests and QOL was found. In conclusion, this study shows that sarcoidosis has a considerable impact on the QOL of patients. The WHOQOL-100 appeared to be a sensitive instrument to measure fatigue - one of the most common symptoms in sarcoidosis - which otherwise is difficult to assess objectively.

\section{Introduction}

Over the past two decades, health status and quality of life (QOL) have been studied for a considerable number of chronic disease conditions [1] including respiratory diseases such as asthma [2] and chronic obstructive pulmonary disease (COPD) [3-9]. COPD has been associated with impaired QOL [4,6], a high incidence of depression $[3,5,6]$ and anxiety $[6]$. However, the relationship between the physiological parameters of severity of COPD and QOL, when 
present, is not strong [7-9].

With respect to sarcoidosis, no studies regarding health status or QOL were found. Depending on the severity of sarcoidosis and the organ systems involved, patients are asymptomatic or present with symptoms such as cough, dyspnea, chest pain, skin lesions and joint and muscle pain. The wide spectrum of symptoms makes sarcoidosis only partly comparable with other chronic respiratory disorders like COPD. The greatest impact on QOL in sarcoidosis, as seen in the clinical practice, seems to be caused by symptoms such as fatigue and sleeping disorders. Fatigue is a common problem in many chronic disorders [10]. Furthermore, fatigue and sleeping disorders are often associated with depression. With respect to depression in sarcoidosis, research by KLONOFF and KLEINHENZ suggests that sarcoidosis patients as a group do not meet the criteria for clinical depression. Awareness of bodily sensations, however, was found to be associated with more depressive symptoms and avoidance of activities when alone [11].

In sarcoidosis, QOL and the feature fatigue have not been studied. Therefore, the primary objective of this study was to evaluate the QOL of sarcoidosis patients. A broad-ranging QOL instrument, the WHOQOL-100, which also measures fatigue, was employed. A secondary objective of this study was to investigate whether fatigue and sleeping problems in sarcoidosis patients are related to depressive symptoms.

\section{Materials and methods}

\section{Patients}

The patients suffering from sarcoidosis from eight participating Dutch hospitals (Rijnstate Hospital, Arnhem; Academic Hospital St Radboud, Nijmegen; Rehabilitation Centre Dekkerswald, Nijmegen; District Hospital Middle Twente, Hengelo; St. Jans-Hospital, Weert; Maasland Hospital, Sittard; The Wever Hospital, 
Heerlen; and University Hospital, Maastricht) were studied. The diagnosis of sarcoidosis was based on consistent clinical features, along with biopsy evidence of noncaseating epitheloid cell granulomas. The clinical symptoms of the respective patients varied from none (sarcoidosis detected on routine chest $X$-ray film) to respiratory and general constitutional symptoms or erythema nodosum and arthralgia (i.e. Löfgren's syndrome). None of the participating subjects had any medical history, which might influence the quality of life. Moreover, patients with co-morbidity were excluded. None of the patients had any anti-depressant pharmacotherapy. In order to be able to evaluate the possible impact of the reported symptoms, as well as to evaluate the quality of life in patients without reported symptoms, the patients were divided into two groups and were studied separately. Group I consisted of patients with current symptoms $(n=37)$, whereas group II consisted of the patients without current symptoms $(\mathrm{n}=27)$. The mean ( $\mathrm{sD}$ ) time from diagnosis for the whole group of sarcoidosis patients was $5.0(5.5)$ years. For demographic characteristics of the patients see table I.

Table I. - Demographic characteristics of the studied sarcoidosis patients with separate data for patients with (group I) and without (group II) current symptoms and the control group

Sarcoidosis patients

Control group

Total group Group I Group II

\begin{tabular}{lllll}
\hline Number of cases & 64 & 37 & 27 & 64 \\
Gender (male/female) & $37 / 27$ & $21 / 16$ & $16 / 11$ & $37 / 27$ \\
Age* & $42.7 \pm 11.9$ & $45.4 \pm 12.8$ & $39.0 \pm 9.6$ & $42.8 \pm 12$ \\
Smoking (yes/no) & $11 / 53$ & $8 / 29$ & $3 / 24$ & \\
\hline
\end{tabular}

* Data are expressed in mean $\pm \mathrm{SD}$. 


\section{Procedure}

Seventy-one sarcoidosis patients from the eight participating hospitals were contacted. Seven patients declined for various private reasons, but 64 patients agreed to participate after signing an informed consent. These sarcoidosis patients were studied. They completed the WHOQOL-100 and the Beck Depression Inventory (BDI). In addition, the physician asked patients about their smoking habits and whether they had any of the following symptoms: fatigue, dyspnea, cough, arthralgia and/or erythema nodosum.

The values of the assessed pulmonary function tests were obtained from the patient record, from the visit closest to the session the questionnaires were completed.

The healthy control group was recruited in the following way: 528 persons selected at random from the zipcode book were called. Of these persons 240 indicated to be willing to participate in a study on QOL. From the group of persons who returned a completed test-booklet $(n=178 ; 74.2 \%$ ), matched healthy control subjects ( $n=64)$ were selected on the basis of gender and age (table I). These control persons were used in order to compare their WHOQOL-100 scores with those of the sarcoidosis patients; they did not complete the Beck Depression Inventory (BDI). The control group was divided into two groups in such a way that patients with and without current symptoms had their own matched control group. The rationale for splitting the control group was that both groups of sarcoidosis patients would have their own matched controls in case the results showed that patients with current symptoms scored significantly different with respect to QOL from with the patients without current symptoms.

Both questionnaires, the WHOQOL-100 and the BDI are self-report measures. After the subjects had completed these questionnaires, a study assistant checked whether the questionnaires had been filled in completely and no questions were skipped.

\section{Pulmonary function tests}

Pulmonary function measurements included the forced expiration volume in one second (FEV $)_{1}$ (Compactbody, Jaeger, Würzburg, Germany). The best measure of 
three efforts was selected. All volumes are expressed as percentages of the reference values [12]. The transfer factor of the lung for carbon monoxide $(T, C 0)$ was measured using the single breath method (Masterlab, Jaeger, Würzburg, Germany).

\section{Modifications in $F E V_{1}$, and $T \mathrm{~L}, \mathrm{CO}$}

In order to compare the $\mathrm{FEV}_{1}$ and $T_{\mathrm{L}}$, co levels with the quality oflife scores, the values were classified according to the American Medical Association classes: 1) nomal range $(\geq 80 \%) ; 2$ ) mill decrease $(60-80 \%) ; 3)$ moderate decrease $(40-60 \%)$ and 4$)$ severe decrease $(<40 \%$ ) predicted, respectively.

\section{Questionnatres}

World Health Organization Quality of Life assessment instrument (WHOQOL-100) The WHOQOL-100 (Dutch version) [13] is a cross-culturally developed generic multidimensional QOL measure that has been simultaneously developed in 15 centres around the world, such as in France, Russia, USA, Panama, Zimbabwe, Japan, Thailand, and The Netherlands [14]. The World Health Organization Quality of Life group (WHOQOL group) has defined QOL as "an individual's perception of his/her position in life in the context of the culture and value systems, in which he/she lives and in relation to their goals, expectations, standards, and concerns" [14].

The WHOQOL-100 consists of 100 items assessing 24 facets of QOL within six domains (physical health, psychological health, level of independence, social. relationships, environment, and spirituality/religion/personal beliefs) and a general evaluative facet (overall quality of life and general health). Table II gives the questions from the facets energy and fatigue and sleep and rest from the domain physical health. Each facet is represented by four items. The response scale is a five-point Likert scale [14]. Except for the facets pain and discomfort, negative feelings, and dependence on medication or treatments, higher scores show a better QOL for the facet or domain. Before calculating the domain scores the scores of these three facets have to be reversed. The reliability and validity of the Dutch version of the WHOQOL are high 
[15]. For instance, the Cronbach alphas for the separate facets range from 0.71 to 0.94 and the questionnaire makes clear distinctions between healthy and chronically ill subjects.

Table II. - WHOQOL items from the facets energy and fatigue and sleep and rest from the domain physical health

Facet 'energy and fatigue' How easily do you get tired?

How much are you bothered by fatigue?

Do you have enough energy for every day life?

How satisfied are you with the energy you have?

Facet 'sleep and rest' Do you have any difficulties sleeping?

How much do any sleep problems worry you?

How satisfied are you with your sleep?

How well do you sleep?

\section{Beck Depression Inventory (BDD)}

Depressive symptoms of the sarcoidosis patients were measured with the BDI [16]. This instrument consists of statements which are grouped in 21 groups of four possible responses. Each answer is scored on a four-point Likert-type scale of 0-3. In previous studies internal consistency was mostly higher than 0.85. Bosscher et al. found scores ranging from 4.5 to $5.0 \mathrm{in}$ a non-clinical student population [17]. A patient with a total score of 15 or above was considered to have significant depressive symptoms [5]. In order to control for the physical effects of the illness on mood, items referring to problems with fatigue, sleep, sexual dysfunction, eating, weight, and general health were summed and labelled the physical depression index (PDI). The remaining items were summed and labelled the cognitive depression index (CDI). Peterson et al. showed that this was a valid way of preventing spurious relationships in a population with end-stage renal disease [18]. Internal consistency of the CDI in this sample was high $(\alpha=0.85)$. 


\section{Statistical analysis}

Data are expressed as mean \pm SD and, if appropriate, as a mean with its range. In order to detect statistically significant differences between the patient groups and their control groups, data were analyzed for the WHOQOL-100 with Student's $t$-tests.

The significance concerning personal characteristics was tested using Chi-squared tests for categorical data and Student's $t$-tests for continuous data. Due to the number of facets in the WHOQOL-100 and thus the number of analyses examined, a probability value of less than 0.01 was considered to be statistically significant. All analyses were performed using the Statistical Package for the Social Sciences (SPSS) [19].

\section{Results}

Patients with current symptoms ( $n=37$; group I) had also had more symptoms at the time of the initial presentation of sarcoidosis than those without current symptoms ( $\mathrm{n}=27$; group II).

The reported actual symptoms of the group of symptomatic patients were fatigue in 28 cases, dyspnea in 15 cases, arthralgia in 11 cases, cough in six cases, and erythema nodosum in one. In line with this, the major symptom assessed by the WHOQOL-100 domain physical health also appeared to be fatigue. The QOL of the patients suffering from sarcoidosis was scrutinized for the WHOQOL-100 domains and their facets, separately. Age was related to lower scores on the facet transport $(r=0.37, p<0.01)$ and higher scores on the facet dependence on medication or treatments $(r=0.34, p<0.01)$.

Symptomatic patients $(n=37)$ scored significantly different from the asymptomatic patients with respect to the domain level of independence (equal variance $t$-value $4.28, \mathrm{p}<0.001$ ) and the facets mobility (equal variance $t$-value $3.15, \mathrm{p}<0.01$ ), activities of daily living (equal variance $t$-value $3.88, \mathrm{p}<0.01$ ), 
Table III. - WHOQOL-100 scores of the studied sarcoidosis patients with separate data for the patients with (group I) and without (group II) current symptoms and the scores for the total control group

\begin{tabular}{|c|c|c|c|}
\hline WHOQOL-100 scores & $\begin{array}{l}\text { Group I } \\
(\mathrm{n}=37)\end{array}$ & $\begin{array}{c}\text { Group II } \\
(n=27)\end{array}$ & $\begin{array}{l}\text { Contral group } \\
\qquad(n=64)\end{array}$ \\
\hline Overall quality of life and general health & $14.2(3.7)$ & $14.2(3.7)$ & $16.0(2.5)^{s}$ \\
\hline Playsical health & $14.3(2.8)$ & $14.2(3.4)$ & $16.0(1.9)^{:}$ \\
\hline Pain and discomfort & $9.2(3.1)$ & $9.1(3.8)$ & $9.0(2.9)$ \\
\hline Energy and fariguc & $12.2(3.7)$ & $12.0(4.5)$ & $15.3(2.5)^{*}$ \\
\hline Sleep and rest & $14.8(3.8)$ & $16.9(3.6)$ & $17.6(2.4)^{8}$ \\
\hline Psychologicall health & $14.5(2.6)$ & $16.1(2.0)$ & $15.3(1.7)$ \\
\hline Positive feelings ${ }^{\dagger}$ & $13.8(3.0)$ & $15.7(2.2)$ & $14.6(1.8)$ \\
\hline Thinking, learning, memory, and concentration & $14.0(3.4)$ & $16.1(2.9)$ & $14.8(2.3)$ \\
\hline Self-esteem & $13.9(3.0)$ & $15.2(2.4)$ & $14.7(2.0)$ \\
\hline Body image and appearance & $16.1(3.5)$ & $17.2(2.2)$ & $17.0(2.5)$ \\
\hline Negative feelings & $9.1(3.1)$ & $7.8(3.0)$ & $8.6(2.9)$ \\
\hline Level of independence ${ }^{\star}$ & $13.9(3.3)$ & $17.2(2.7)$ & $17.4(2.4)$ \\
\hline Mobility & $15.5(3.4)$ & $18.0(2.4)$ & $17.3(3.2)$ \\
\hline Activities of daily livingt & $13.4(3.7)$ & $16.8(3.3)$ & $17.0(2.3)$ \\
\hline Dependence on medication and trestments ${ }^{t}$ & $10.0(4.2)$ & $6.8(3.2)$ & $5.8(2.9)$ \\
\hline Working capacity ${ }^{*}$ & $12.8(4.2)$ & $17.0(3.3)$ & $17.3(2.8)$ \\
\hline Social relationships & $15.4(2.8)$ & $16.9(2.5)$ & $15.6(2.2)$ \\
\hline Personal relationships & $15.7(3.0)$ & $17.1(28)$ & $16.1(2.0)$ \\
\hline Social support & $15.4(3.6)$ & $17.2(2.8)$ & $15.8(2.4)$ \\
\hline Sexual activity & $15.2(3.3)$ & $16.3(2.9)$ & $14.6(3.1)$ \\
\hline Environment & $15.6(2.1)$ & $16.9(1.9)$ & $15.6(1.7)$ \\
\hline Physical safety and security & $15.7(2.8)$ & $16.9(2.8)$ & $15.5(2.0)$ \\
\hline Home environment & $15.5(2.9)$ & $17.2(2.9)$ & $15.6(2.6)$ \\
\hline Financial resources & $16.5(3.2)$ & $17.3(2.3)$ & $15.7(3.1)$ \\
\hline Health and social care: availability and quality & $15.1(2.1)$ & $16.0(2.7)$ & $14.8(2.2)$ \\
\hline Ability to acquire new information and skills & $15.5(2.8)$ & $16.3 \cdot(2.5)$ & $15.5(2.4)$ \\
\hline Participating in and possibilities for recreation/leisure & $14.3(3.8)$ & $16.8(2,9)$ & $15.7(2.7)$ \\
\hline Physical environment (pollution' nolse/ traffic/ climate) & $14.7(2.8)$ & $15.6(2.6)$ & $14.9(2.2)$ \\
\hline Transport & $17.3(3.4)$ & $18.9(1.7)$ & $17.3(3.0)$ \\
\hline Spirituality/religion/personal beliefs & $12.8(3.5)$ & $13.7(3.4)$ & $12.7(2.7)$ \\
\hline
\end{tabular}

Note: Student"s $t$-tests were applied. Data are expressed as mean with so in parentheses. Group 1 ws. group II: * $\mathrm{p}<0.001,{ }^{\dagger} \mathrm{p}<0.01$. Total patient group (scores not shown) vs. total control group: $p<0.001,{ }^{5} \mathrm{p}<0.01$. 
dependence on medication or treatments (equal variance $t$-value $-3.29, \mathrm{p}<0.01$ ), working capacity (unequal variance $t$-value $4.55, \mathrm{p}<0.01$ ), positive feelings (equal variance $t$-value $2.79, \mathrm{p}<0.01$ ), and participating in and possibilities for recreation/leisure (equal variance $t$-value $2.82, \mathrm{p}<0.01$ ). In each case, the $\mathrm{QOL}$ of the patients with symptoms was lower than the QOL of the patients without symptoms (table III).

For these domains and facets, separate analyses were done for patients with symptoms and matched healthy control subjects, and patients without symptoms and their matched healthy control subjects, respectively.

Regarding the domain level of independence, the symptomatic patients scored lower (unequal variance $t$-value $5.24, d f 64.4, \mathrm{p}<0.001$ ) than their control group. At facet level, these patients scored lower on activities of daily living (unequal variance $t$-value 5.43 , df $59, \mathrm{p}<0.001$ ), and working capacity (unequal variance $t$-value 5.04 , df $64.1, \mathrm{p}<0.001$ ), and higher on the facet dependency on medication or treatments (unequal variance $t$-value $-5.12, d f$ $62.9, \mathrm{p}<0.001)$ than their matched healthy control subjects.

Patients without symptoms scored significantly different from their control subjects on the domain environment (equal variance $t$-value -2.95 , df 52 , $\mathrm{p}<0.01$ ) and its facets home environment (equal variance $t$-value -2.85 , df 52 , $\mathrm{p}=0.01$ ) and transport (equal variance $t$-value -2.75 , df $52, \mathrm{p}<0.01$ ). With respect to these latter areas, patients scored higher than the healthy persons.

Regarding the other domains and facets, the results for symptomatic and asymptomatic patients were comparable. Therefore, the patients were treated as one group when their scores on these QOL domains and facets were compared with the matched healthy controls.

Differences between the total sarcoidosis patient group and the total healthy control group, emerged on the domain physical health (unequal variance $t$ value $3.79, d f 102.7, \mathrm{p}<0.001$ ) and its facets energy and fatigue (unequal variance $t$-value $5.30, d f 101, \mathrm{p}<0.001$ ) and sleep and rest (unequal variance $t$ value $3.38, d f 105.8, \mathrm{p}=0.001$ ). In general, the sarcoidosis group indicated that 
they felt physically less healthy and their general sense of QOL was lower.

With respect to depressive symptoms, respondents with current symptoms had significantly higher BDI scores $(9.8 \pm 7.8)$ than those without symptoms

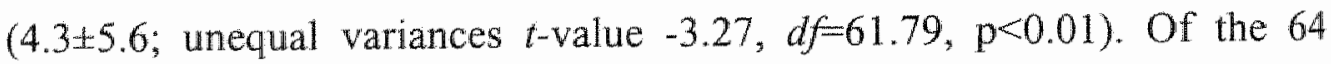
patients, $12(18 \%)$ had a BDI score of 15 or above (associated with significant depression) [5]. Of those 12 cases only one belonged to the group of patients

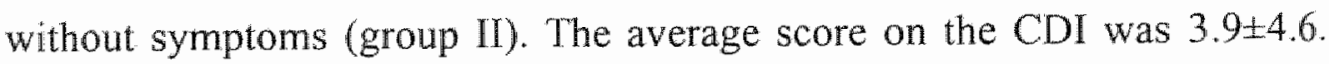

Table IV. - Medical characteristics of the studied sarcoidosis patients with (group I) and without (group II) current symptoms

\begin{tabular}{lll}
\hline & Group I $(n=37)$ & Group II $(n=27)$ \\
\hline $\begin{array}{l}\text { Duration of disease, years } \\
\text { FEV } \\
>80 \%\end{array}$ & $4.4 \pm 5.1$ & $6.1 \pm 5.9$ \\
$60-80 \%$ & 29 & 23 \\
$T_{L}, \mathrm{CO}$ & 8 & 4 \\
$>80 \%$ & & 24 \\
$60-80 \%$ & 22 & 3 \\
$40-60 \%$ & 12 & - \\
\hline
\end{tabular}

$\mathrm{FEV}_{1}$ : forced expiration volume in one second; $T \mathrm{~L}, \mathrm{CO}$ : transfer factor of the lung for carbon monoxide. ${ }^{*}$ Data are expressed in mean $\pm \mathrm{SD} .{ }^{\dagger} \mathrm{p}<0.01$ group I $v$. group II.

Univariate analysis still showed that symptomatic patients had higher CDI scores $(5.2 \pm 5.3)$ than those without symptoms $(2.2 \pm 3.6 ; p<0.01)$.

For the WHOQOL-100 only the domain psychological health and the facet fatigue were related to depression $(p<0.01)$. No correlation between the facet fatigue and the domain psychological health was found.

\section{Pulmonary function tests}

Pulmonary function tests of the respective patients groups are summarized in table IV. No correlations between the WHOQOL-100 scores and the pulmonary function tests were found. 


\section{Discussion}

The results of this study showed that the major problems of patients with sarcoidosis are fatigue and low levels of energy, and that these symptoms can be measured in a standardized format.

Surprisingly, both patient groups - including patients who had reported no current symptoms - suffered from fatigue, sleeping problems and impaired general quality of life compared to the healthy control group. Sarcoidosis patients who had considered themselves asymptomatic also demonstrated an impaired QOL.

A number of differences in QOL were found between the patient groups with and without current symptoms. Besides the physical problems mentioned above, patients with current symptoms suffered from impaired QOL mainly with respect to the level of independence. This area includes problems with patients' mobility, working capacity, and activities of daily living. Moreover, group I had low levels of positive feelings and problems with recreation compared with group II. The fact that symptomatic sarcoidosis patients had a poor QOL compared to asymptomatic patients is not surprising. However, the areas in which QOL of these patients were impaired indicates that sarcoidosis has a considerable impact on daily life, even in patients with a relatively mild impairment of pulmonary function tests.

Although in many disorders fatigue is a common problem [10], in sarcoidosis fatigue and loss of energy have had hardly any attention in the literature. In the present study, scores for fatigue and loss of energy were found to be similar to those of a patient population suffering from rheumatoid arthritis [20].

Depressed mood and clinical depression are known to be associated with a lower QOL [3,5,18]. In agreement with KLONOFF et al., the sarcoidosis patients as a group did not meet the criteria for clinical depression [11]. However, 
patients with current symptoms had higher BDI and CDI scores than asymptomatic patients. For the WHOQOL-100 only the domain psychological health was associated with depression. This indicates that patients who were less satisfied with their psychological situation were more depressed. The facet fatigue was related to depression, but not to the domain psychological health.

The association found between the psychological health of patients and depressive symptoms could be expected, irrespective of the impairment of sarcoidosis. The absence of an association between fatigue and psychological health, however, suggests that the relationship between fatigue and depression can be explained in the context of the ongoing sarcoidosis. Patients may become exhausted by the disease. Depressive symptoms, then, at least partly, are the psychological expression of exhaustion.

For several years now, evidence has accumulated that patients with depressive disorders experience more limitations in well-being and social functioning, when compared with healthy controls and patients with chronic illness. The combination of chronic illness and depressive symptoms, however, is associated with increased morbidity and impairment of social functioning [21]. Recent research suggests that functional ability plays a role here. In chronic obstructive pulmonary disease (COPD), mood and attitude were found to be closely related to breathlessness [22]. Kellner et al. found depressive symptoms to be associated with self-rated pulmonary complaints (i.e. breathlessness) [23]. COPD patients with greater distress and poorer coping had a higher probability of readmission to hospital independent of disease severity assessed by pulmonary function tests [24]. In COPD, treating psychosocial aspects and improving the patient's ability to cope with the disease has been found to be an important aspect [6]. In sarcoidosis, teaching patients to cope with the limitations implied by the disease, offering psychosocial support and treating depressive symptoms may also be important. Intervention research is needed to study the causal relationship between complaints and psychological factors. 
No relationship between pulmonary function tests and QOL was found. These results suggest that pulmonary function tests may not be sensitive parameters for QOL in sarcoidosis. Previously, we found that health status assessed by the Sickness Impact Profile [25] did not correlate with routinely performed pulmonary function tests in a sub-population of this patient group [26]. Moreover, the majority of patients in the present study had a normal $\mathrm{FEV}$, and $T \mathrm{~L}, \mathrm{co}$. The limitations of this patient group, therefore, do not seem to be attributable to abnormalities in ventilatory mechanics. In our previous study mentioned above, we also found that respiratory muscle strength and endurance time was decreased in sarcoidosis patients compared to healthy controls. Moreover, a relationship was found between decreased respiratory muscle endurance time and the presence of symptoms such as fatigue [26]. Future studies should focus on, in addition to respiratory symptoms, constitutional complaints such as fatigue and sleeping problems which are otherwise difficult to assess objectively.

One limitation of this study is the rather low number of patients involved, which reduces the statistical power.

In conclusion, this study shows that sarcoidosis, especially in symptomatic patients, has a considerable impact on quality of life. Rather vague symptoms like fatigue and loss of energy as well as sleeping problems in patients with sarcoidosis can be assessed with the WHOQOL-100. Moreover, the symptom fatigue was not associated with psychological health and should be attributed to the ongoing disease. Psychological health was associated with depressive symptoms in sarcoidosis patients. Treating depressive symptoms and including psychological interventions in sarcoidosis patient care may improve QOL in these patients. Intervention research, however, is needed here. 


\section{Acknowledgements}

This paper is based on data and experience obtained during the WHO Project on the development of the World Health Organization Quality of Life assessment instrument. The authors would like to thank Leonie Kock and Sylvia Drent for gathering the data and Marjon Elfferich for her valuable comments during the preparation of the manuscript.

\section{References}

1. Patrick DL, Deyo RA. Generic and disease-specific measures in assessing health status and quality of life. Med Care 1989;27: S217-S232.

2. Juniper EF, Guyatt GH, Ferrie PJ, Griffith LE. Measuring quality of life in asthma. Am Rev Respir Dis 1993; 147: 832-838.

3. McSweeny AJ, Grant I, Heaton RK, Adams KM, Timms RM. Life quality of patients with chronic obstructive pulmonary disease. Arch Intern Med 1982; 142 : 473-478.

4. Guyatt GH, Townsend M, Berman LB, Pugsley SO. Quality of life in patients with chronic airflow limitation. Br J Dis Chest 1987; 81:45-54.

5. Light RW, Merrill EJ, Despars JA, Gordon GH, Mutalipassi LR. Prevalence of depression and anxiety in patients with COPD. Relationship to functional capacity. Chest 1985; 87: 35-38.

6. Dudley DL, Glaser EM, Jorgenson BN, Logan DL. Psychosocial concomitants to rehabilitation in chronic obstructive pulmonary disease. Part I. Psychosocial and psychological considerations. Chesi 1980; 77:413-420.

7. Schrier AC, Dekker FW, Kaptein AA, Dijkman JH. Quality of life in elderly patients with chronic nonspecific lung disease seen in family practice. Chest 1990; 98: 894-899.

8. Williams SJ, Bury MR. Impairment, disability and handicap in chronic respiratory illness. Soc Sci Med 1989; 29: 609-616.

9. Curtis JR, Deyo RA, Hudson LD. Health-related quality of life among patients with chronic obstructive pulmonary disease. Thorax 1994; 49: 162-170.

10. Thomas PK. The chronic fatigue syndrome: what do we know? Br Med J 1993; 306: 1557-1558.

11. KlonoffEA, Kleinhenz ME. Psychological factors in sarcoidosis: the relationship between life stress and pulmonary function. Sarcoidosis 1993; 10: 118-124.

12. Quanjer PH, Tammeling GJ, Cotes JE, Pederson OF, Peslin R, Yernault J-C. Lung volumes and forced ventilatory flows. Report of the working party. Standardization of lung function tests. European Commission for Steel and Coal. Official statement of the European Respiratory Society. Eur Respir $J 1993 ; 6$ (suppl 16): 5-40. 
13. WHOQOL group. Field trial WHOQOL-100 February 1995 : facet definitions and questions. 1995a; Geneva: WHO (MNH/PSF/95.1.B).

14. WHOQOL group. Development of the WHOQOL: rationale and current status. Int $J$ Ment Health 1994; 23 : 24-56.

15. De Vries J, Van Heck GL. The World Health Organization Quality of Life assessment instrument (WHOQOL-100): validation study with the Dutch version. Eur J Psychol Assessment 1997; 13: 164-178.

16. Beck AT, Ward CH, Mendelson M, Mock J, Erbaugh J. An inventory for measuring depression. Arch Gen Psychiat 1961; 4: 561-571.

17. Bosscher RJ, Koning H, Van Meurs R. Reliability and validity of the Beck Depression Inventory in a Dutch college population. Psychol Reports $1986 ; 58$ : 696-698.

18. Peterson RA, Kimmel PL, Sacks CR, Mesquita ML, Simmens SJ, Reiss D. Depression, perception of illness and mortality in patients with end-stage renal disease. Int J Psychiat Med 1991; 21: 343-354.

19. SPSS-PC for the IBM PC/XT/AT. SPSS Inc., Chicago, 1986.

20. De Vries J. Beyond health status. Construction and Validation of the Dutch WHO Quality of life assessment instrument. Doctoral thesis, Tilburg University, Tilburg, The Netherlands, 1996.

21. Klerman, GL. Depressive disorders: further evidence for increased medical morbidity and impairment of social functioning. Arch Gen Psychiatry 1989; 46: 856-858.

22. King B, Cotes JE. Relation of lung function and exercise capacity to mood and attitudes to health. Thorax 1989; 44:402-409.

23. Kellner R, Samet J, Pathak D. Dyspnea, anxiety, and depression in chronic respiratory impairment. Gen Hosp Psychiat 1992; 14:20-28.

24. Osman LM, Godden DJ, Friend JAR, Legge JS, Douglas JG. Quality of life and hospital re-admission in patients with chronic obstructive pulmonary disease. Thorax 1997; 52: 67-71.

25. Bergner M, Bobbit RA, Carter WB, Gilson BS. The Sickness Impact Profile: development and final revision of a health status measure. Med Care 1981; 19: $787-805$

26. Wirnsberger $R M$, Drent $M$, Hekelaar $N$, et al. Relationship between respiratory muscle function and quality of life in sarcoidosis. Eur Respir $J 1997 ; 10$ : 14501455. 
Chapter 6

Impairment of quality of life: rheumatoid arthritis versus sarcoidosis 


\title{
Impairment of quality of life: rheumatoid arthritis versus sarcoidosis
}

\author{
R.M. Wirnsberger*, J. de Vries**, T.L.Th.A. Jansen***, \\ G.L. van Heck**, E.F.M. Wouters* and M. Drent*
}

*Department of Pulmonology, University Hospital, Maastricht, ** Department of Psychology, Tilburg University, Tilburg, and *** Department of Rheumatology, Medical Centre Leeuwarden, Leewwarden, The Netherlands

Neth J Med: accepted for publication 


\section{Abstract}

Quality of life (QOL) has become an important item in health care. QOL should be a major target of treatment in chronic diseases such as rheumatoid arthritis (RA) and sarcoidosis. The aim of this study was to compare the impact of RA and sarcoidosis on patients' QOL. We expected more serious impairment of QOL in the RA group than in the sarcoidosis group. QOL was studied in RA patients ( $n=32)$, sarcoidosis patients ( $n=37)$, and a healthy control group $(n=37)$ employing the World Health Organization Quality of Life assessment instrument (WHOQOL-100). In both patient groups QOL was impaired with respect to physical health, level of independence $(\mathrm{p}<0.001)$ and overall QOL and general health $(\mathrm{p}<0.01)$. Moreover, RA patients appeared to have a lower QOL with respect to pain and discomfort $(p<0.001)$ and mobility $(p<0.001)$. In conclusion, in RA and sarcoidosis, fatigue and sleep were major problems. In contrast to our expectations, with respect to activities of daily living and working capacity, the two patient groups did not show any difference. Impairment of QOL was more serious and included more aspects of QOL in RA than in sarcoidosis.

\section{Introduction}

In the past two decades, assessment of health status and quality of life (QOL) as an outcome measure has become increasingly important in patients with chronic diseases, including rheumatoid arthritis (RA) [1-5]. Improvement of patients' QOL has become a major target of treatment. In general, health status and QOL of RA patients are substantially impaired, whereas disease status as assessed by disease related variables does not reflect accurately the impact of the disease on psychological and social well-being $[4,6]$. In sarcoidosis, health 
status and QOL have had little attention. Recently, we found impairment of health status and QOL in sarcoidosis patients $[7,8]$. No relationship of routinely performed pulmonary function tests and serum angiotensin converting enzyme (SACE) with health status and QOL was found $[7,8]$.

Sarcoidosis and RA have several sequelae in common. Both are inflammatory disorders of unknown etiology. In both disorders the mode of onset is variable and they both regularly occur in relatively young people. The clinical course in both disorders is rather unpredictable, ranging from spontaneous remission to rapid progression or a more chronic course. Some immunologic disturbance is thought to play a central role in the pathogenesis of sarcoidosis [9] as well as in RA [10]. Manifestations of RA are primarily in the joints, but systemic symptoms and extra-articular inflammation may be present as well. In RA, as in sarcoidosis, pulmonary involvement with interstitial pneumonitis or fibrosis, however only occasionally, may occur $[11,12]$. In sarcoidosis, symptoms vary considerably depending on the organ involvement. Patients with sarcoidosis may present with aspecific symptoms also present in RA such as fatigue, arthralgia, and muscle pain. In a previous study, we found that in about $5 \%$ of patients with sarcoidosis, the symptoms were initially attributed to RA [13]. Finally, in both disorders no specific causal treatment is known at present.

In rheumatology a number of heal th status measures have been used to assess QOL [4]. Recently, RUTA et al. validated the SF-36, a generic health status measure, in RA patients [14]. However, usually, disease impact of RA on patients' lives has been assessed rather by disease-specific health status measures [5,15-17] than by subjective QOL measures. Health status measures assess mainly functional status, whereas QOL instruments assess the individuals' subjective perception of their life. When using health status measures, it would be a mistake to equate lower levells of functioning with lower QOL. For this conclusion would contrast with findings in QOL research reflecting high perceived QOL in spite of low levels of functioning [18]. 
To the best of our knowledge, there exist no comparative studies on QOL in RA and other chronic inflammatory disorders such as sarcoidosis. Therefore, the aim of this study was to compare the impact on QOL of RA and sarcoidosis. Recently, we showed that QOL in symptomatic sarcoidosis patients was more seriously impaired than QOL in patients without current symptoms [8]. We hypothesized that QOL would be impaired in both disorders compared to healthy controls. Sarcoidosis patients were expected to have better QOL than RA patients. Especially with respect to pain and levels of independence we expected a more serious impairment of QOL in the RA patients. With respect to fatigue no difference between the patient groups was expected. Therefore, in the present study QOL in active RA was compared with QOL of symptomatic sarcoidosis patients. For this purpose a broad-ranging generic QOL instrument, the World Health Organization Quality of Life assessment instrument (WHOQOL-100) [19], was used.

\section{Materials and methods}

\section{Patients}

A group of RA patients $(n=35)$ was recruited aselectively from consecutive patients with RA not in remission visiting the Outpatient Department of Rheumatology, Rijnstate Hospital, Arnhem. When asked to participate in the study, three patients refused for various reasons, whereas 32 patients agreed to participate in the study. None of those latter patients had any co-morbidity. The diagnosis of RA was based on the American College of Rheumatology (formerly the American Rheumatism Association) criteria for RA [20]. The patients were clinically assessed by a rheumatologist, who completed a questionnaire with the clinical characteristics: disease duration, functional class according to Steinbrocker [21], current serum markers (erythrocyte sedimentation rate (ERS) and C-reactive protein (CRP)), 28 swollen joint index, and current medication. At the same visit the patient completed 
the WHOQOL-100 [19].

The sarcoidosis patient group was recruited from eight Dutch hospitals (Rijnstate Hospital, Arnhem; Academic Hospital St Radboud, Nijmegen; Rehabilitation Center Dekkerswald, Nijmegen; District Hospital Middle Twente, Hengelo; St. JansHospital, Weert; Maasland Hospital, Sittard; The Wever Hospital, Heerlen; and University Hospital, Maastricht). Seventy-one unselected consecutive out-patients with sarcoidosis, without any co-morbidity were contacted. Seven sarcoidosis patients declined for various reasons, but 64 agreed to participate. The diagnosis of sarcoidosis was based on clinical findings, along with histological evidence. None of the patients had any previous medical condition which might have influenced their QOL. All patients completed the WHOQOL-100. In addition, the physician asked them whether they had any of the following complaints: fatigue, dyspnea, coughing, arthralgia or erythema nodosum. Twenty-seven out of the 64 studied sarcoidosis patients reported no actual symptoms, whereas 37 patients appeared to suffer form one or more symptom(s). In order to be able to compare the impact of symptoms between sarcoidosis patients and the studied RA patients, who were not in remission, only sarcoidosis patients with current symptoms related to their disorder $(n=37)$ were included in the present study. Using the patient record, the values of serum angiotensin converting enzyme (SACE) levels and the pulmonary function tests were obtained from the visit closest to the session in which the questionnaires were completed.

Table I. - Characteristics of the studied sarcoidosis patients, rheumatoid arthritis (RA) patients, and the healthy control group

Variable

RA patients Sarcoidosis patients Control group

Number

Age, years *

Gender male/female
32

$54.3(29-70)$

$13 / 19$
37

$45.3(26-73)$

$21 / 16$
37

45.4 (26-73)

$21 / 16$

* Data are means with the range in parentheses 
Furthermore, 240 control persons were recruited who were willing to participate in a study on QOL. From the group of persons who returned a completed test-booklet $(n=178 ; 74.2 \%)$, healthy control subjects $(n=37)$ were selected on the basis of gender and age, matched for the sarcoidosis patients. For demographical characteristics of the two patient groups and the control group see table I.

The characteristics of the RA patients are summarized in table II. During the previous week five of the RA patients had received systemic corticosteroids; all these patients received prednisolone. One patient was treated with $7.5 \mathrm{mg}$ day-1, two with 10 $\mathrm{mg} \cdot$ day $^{-1}$, one with $15 \mathrm{mg} \cdot \mathrm{day}^{-1}$, and of one patient information on the dose was missing. Only one patient did not receive disease modifying anti-rheumatic drugs (DMARDs) at the time of the study. Ten patients were currently treated with Methothrexate, nine with Sulphasalazine, four with Azathioprine, three with Penicillamine, two with Hydroxychloroquine, one with Auromyosis, and one patient received the combination Sulphasalazine and Metothrexate at the time of the study.

Table II. - Characteristics of the RA patient group

Variable

Disease duration, years*

$8.4(9.3)$

Type RA seropositive/seronegative

$26 / 4$

Steinbrocker I/II/IIL/V

$9 / 22 / 1 / 0$

28 swollen joint index*

$8.3(8.0)$

CRP $\mu \mathrm{g} \cdot \mathrm{mL}^{-1 *}$

$25.9(34.2)$

ESR*

$33.7(25.8)$

Corticosteroid use yes/no

$5 / 27$

Present treatment with DMARDs yes/no

$31 / 1$

Number of DMARDs in the past*

$2.2(2.3)$

* Data are means (sD); CRP: C-reactive protein, normal levels $<9 \mu \mathrm{g} \cdot \mathrm{mL}^{-1}$; ESR: erythrocyte sedimentation rate; DMARDs: disease modifying anti-rheumatic drugs.

The characteristics of the sarcoidosis patients are summarized in table III. The sarcoidosis patients reported the presence of the following symptoms: fatigue in 28 , dyspnea in 15, arthralgia in 11, cough in six patients, and erythema nodosum in one 
patient. With respect to the pulmonary function tests, eight of the sarcoidosis patients $(21.6 \%)$ showed a mildly decreased forced expiration volume in one second $\left(\mathrm{FEV}_{\mathrm{F}}\right)$, and 15 patients showed a decreased $T \mathrm{~L}, \mathrm{CO}$. In 12 of the patients the tranfer factor of the lung for carbon monoxide ( $T \mathrm{~L}, \mathrm{Co}$ ) was mildly decreased and in three patients it was moderately decreased, according to the American Medical Association classes.

\section{Questionnaire}

Both patient groups and the healthy controls completed the World Health Organization Quality of Life assessment instrument, WHOQOL-100, (Dutch version) [19].

Table III. - Characteristics of the sarcoidosis patient group

Variable

Disease duration*

$\mathrm{FEV}_{\text {, (nomal } / \text { decreased) }}$

Th,co (normal/decreased)

SACE U $\cdot \mathrm{L}^{-1}\left(9-25 \mathrm{U} \cdot \mathrm{L}^{-1}\right)^{*}$

Use of corticosteroids (yes/no)
$4.4(5.1)$

$29 / 8$

$22 / 15$

$30(25)$

$14 / 23$

* Data are means (SD); $\mathrm{FEV}_{1}$ : forced expiratory volume in one second; $T \mathrm{~L}, \mathrm{CO}$ : transfer factor of the lung for carbon monoxide; SACE: serum angiotensin converting enzyme.

This is a cross-culturally developed generic multidimensional QOL measure that has been simultaneously developed in 15 centres around the world, e.g., in France, Russia, USA, Panama, Zimbabwe, Japan, Thailand, and The Netherlands [22]. It consists of 100 items assessing 24 facets of QOL within six domains (physical health, psychological health, level of independence, social relationships, environment, and spirituality/ religion/personal beliefs) and a general evaluative facet (overall quality of life and general health). Each facet is represented by four items. The response scale is a fivepoint Likert scale, scores can range from 4-20. Except for the facets pain and discomfort, negative feelings, and dependence on medication and treatment, higher scores mean a better QOL. The reliability and validity of the Dutch version of the instrument are high $[19,23]$. 


\section{Pulmonary function tests}

Pulmonary function measurements of the sarcoidosis patients included the FEV, (Compactbody, Jaeger, Würzburg, Germany). The best measure of three efforts was selected. All volumes are expressed as percentages of the reference values [24]. The $T_{\mathrm{L}}, \mathrm{CO}$ was measured using the single breath method (Master-lab, Jaeger, Würzburg, Germany). Four ranges of $F E V$, and $T_{L}$, co levels were classified according to the American Medical Association classes: 1) normal ( $\geq 80 \%)$; 2 ) mild decrease (60$80 \%)$; 3) moderate decrease $(40-60 \%)$; severe decrease $(<40 \%)$, of the values predicted.

\section{Statistical analysis}

Data are expressed as mean (SD) and, if appropriate, as mean with range. Statistical differences in age and gender between patient groups were studied using Student's $t$ tests and Chi-squared tests. Pearson correlation coefficients were used. Significance was accepted with probability values of less than 0.05 . Furthermore, data were anallyzed using analysis of variance (ANOVA) with age and gender as covariates between groups. Within the RA group one-way analyses of variance were used. In ANOVA, significance was accepted with probability values of less than 0.01 due to the number of analyses. All analyses were performed using the Statistical Package for Social Science (SPSS) for Windows [25].

\section{Results}

The RA patients were significantly older than the sarcoidosis patients $(\mathrm{p}<0.001)$. Table IV summarizes the QOL scores for domains and facets with significant differences between the groups.

Impairment was found with respect to the evaluative facet overall QOL and general health, the domains physical health and its facets, as well as the domain level of independence and its facets, for both patient groups. 
The facets overall QOL and general health $(\mathrm{F}=7.4 ; d f=2, \mathrm{p}<0.01)$, energy and fatigue $(F=16.5 ; d f=2, p<0.001)$, sleep and rest $(F=9.8 ; d f=2, p<0.001)$, activities of daily living $(\mathrm{F}=18.1 ; d f=2, \mathrm{p}<0.001)$, and working capacity $(\mathrm{F}=18.5 ; d f=2, \mathrm{p}<0.001)$ were low in the two patient groups compared to the healthy controls.

Table IV. - WHOQOL-100 domain and facet scores with significant differences between the rheumatoid arthritis (RA) patients, the sarcoidosis patients, and the matched control group

WHOQOL 100

RA Sarcoidosis Control

group group group

Overall quality of life and general health ** Physical health $\$$

$13.6(2.5) \quad 14.2(3.7)$

$16.2(2.3)$

Pain and discomfort

$12.3(2.6) \quad 14.3(2.8)$

$16.1(1.7)$

Energy and fatigue *\$

$12.9(2.7) \quad 9.2(3.1)$

$9.1(2.8)$

Sleep and rest $* \$$

$11.7(3.6) \quad 12.2(3.7)$

$15.8(2.0)$

Level of independence $\$$

$14.0(3.7) \quad 14.8(3.8)$

$17.4(2.5)$

Mobility *

Activities of daily living $*$ s

$12.1(2.9) \quad 13.9(3.3)$

$17.4(2.3)$

$13.6(2.5) \quad 15.5(3.4)$

$17.2(3.3)$

$12.8(3.5) \quad 13.4(3.7)$

$17.2(2.1)$

Dependence on medication or treatments ${ }^{\text {ts }}$

$13.8(3.0) \quad 10.0(4.2)$

$5.7(2.7)$

Working capacity ${ }^{* 5}$

$12.1(3.5) \quad 12.8(4.2)$

$17.0(2.9)$

Analysis of variance (covariates age); scores are means (SD). *Control group scored differently from the two patient groups, ${ }^{\dagger}$ all three groups scored differently from each other, " RA group scored differently from the two other groups, ${ }^{8} \mathrm{p}<0.001, " \mathrm{p}<0.01$. Note: domains are printed in bold; for the facets pain and discomfort and dependence on medication or treatment higher scores mean worse QOL.

In the domains physical health $(F=18.1 ; d f=2, \mathrm{p}<0.001)$ as well as level of independence $(F=26.6 ; d f=2, p<0.001)$ and its facet dependence on medication or treatment $(\mathrm{F}=41.2 ; d f=2, \mathrm{p}<0.001)$ all three groups scored significantly different from each other, the healthy controls having the highest QOL in these 
areas and the RA group the lowest. Furthermore, the group of RA patients scored worse on the facets pain and discomfort $(\mathrm{F}=16.1 ; d f=2, \mathrm{p}<0.001)$ and mobility $(\mathrm{F}=6.9 ; d f=2, \mathrm{p}<0.01$ ) than the sarcoidosis patients and the healthy group.

For the RA patients we found a significant correlation between the evaluative facet overall QOL and general health and the facet pain and discomfort $(r=-0.65, p<0.001)$. Moreover, a relationship was found between the facet energy and fatigue and the facet pain and discomfort $(r=-0.69, p<0.001)$.

四 Class | $\square$ Class I| $\boxminus$ Class III

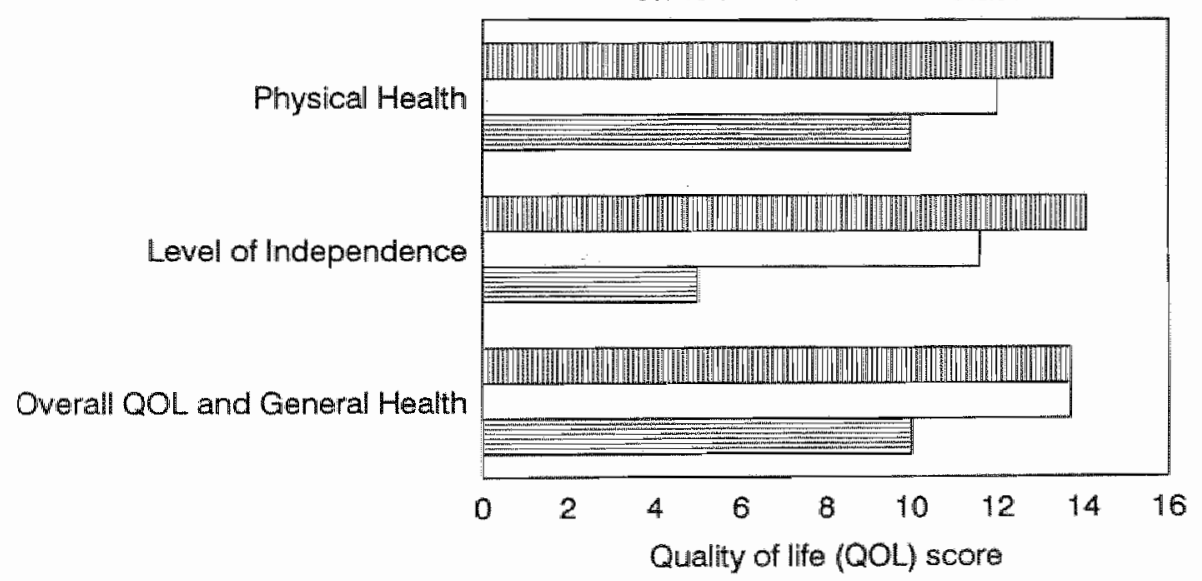

Fig. 1. - Relationships between functional classes and the domains physical health, level of independence, and overall QOL and general health.

(Note, that for the facet pain and discomfort, in contrast to most other facets, higher scores mean a worse QOL). No relationship was found for the facet pain and discomfort with sleep and rest and between the facets energy and fatigue and sleep and rest. Although the RA patients did not show an impairment of their psychological health, the facet energy and fatigue was related to the domain psychological health of the WHOQOL-100 ( $r=0.75, p<0.001$ ). For the sarcoidosis patients no such relationship was found. 
For the RA patients, several relationships were found between the physiological measures of disease severity and QOL. On the domain level, a relationship was found between physical health and the $\operatorname{ESR}(r=-0.50, p<0.01$ ), level of independence and number of courses with DMARDs $(r=-0.35, p=0.05)$, and between environment $(r=-0.42, \mathrm{p}<0.05)$ as well as spirituality/religion/ personal beliefs $(r=-0.41, p<0.05)$ and the doses of systemic corticosteroids.

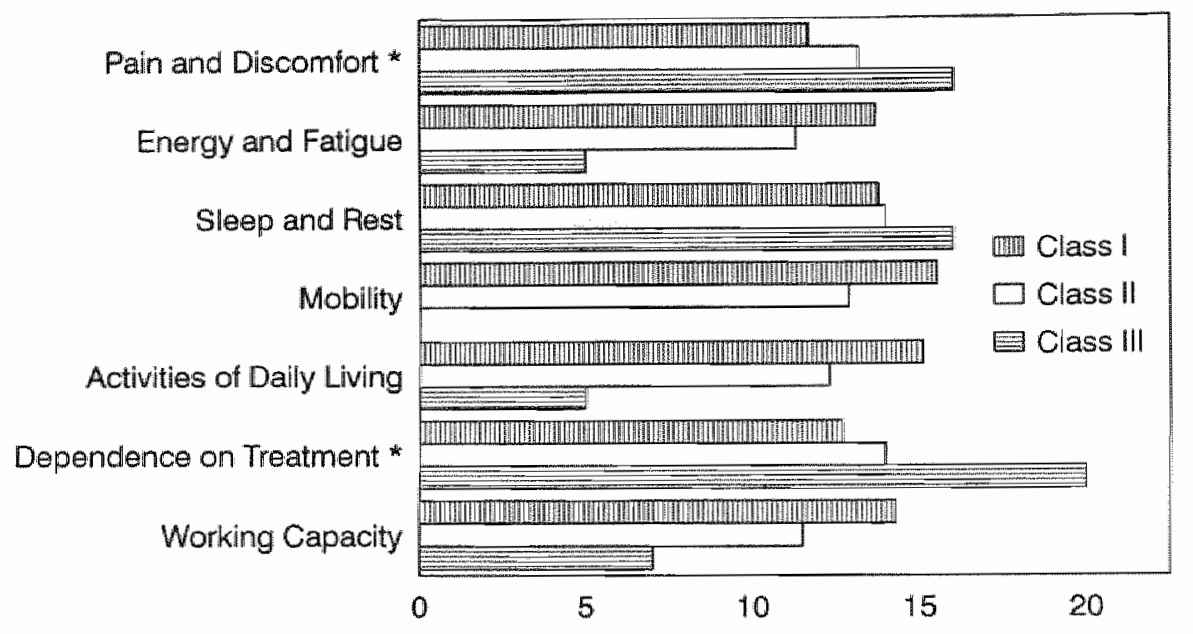

Fig. 2 - Relationships between functional classes and the facets of the domains physical health and level of independence. *High scores indicate a low QOL. Note: the mobility score of functional class III is missing.

On the facet level, energy and fatigue correlated with the number of courses with DMARDs in the past $(r=-0.50, \mathrm{p}<0.01)$, and the doses of systemic corticosteroids $(=-0.39, \mathrm{p}<0.05)$. Moreover, RA patients with elevated CRP levels showed even lower scores for energy and fatigue $(t=2.95, d f=12, \mathrm{p}=0.012)$ than those with normal CRP. The facet sleep and rest correlated with the ESR $(r=-0.54, p<0.001)$. The facets thinking, learning, memory, and concentration 
and bodily image and appearance were related to the swollen joint index ( $1=$ $0.39, \mathrm{p}<0.05$ and $\mathrm{r}=-0.40, \mathrm{p}<0.05$, respectively). Mobility correlated with the $\operatorname{ESR}(r=-0.48, p<0.01)$. Moreover, a relationship was found between activities of daily living and the number of courses of DMARDs $(r=-0.40, p<0.05)$.

The QOL facets ability to acquire new information and skills was related to the number of courses with DMARDs $(r=-0.41, p<0.05)$ and the doses of systemic corticosteroids ( $r=-0.47, \mathrm{p}<0.01$ ). No relationships were found between the QOL facet pain and discomfort and any of the physiological measures of disease severity. In addition, no relationship was found between gender, duration of the disease, and the current medication with DMARDs and patients" QOL. Figure 1 shows the relationship between the functional class and the domains physical health, level of independence, and the evaluative facet overall quality oflife and general health. In figure 2, the relationship between the facets of these two domains and the functional class is illustrated.

The domain psychological health $(\mathrm{F}=5.4, d f=2 ; \mathrm{p}<0.05)$, and its facets selfesteem $(F=9.0, d f=2 ; p<0.001)$ and negative feelings $(F=7.5, d f=2 ; p<0.01)$ were related to positivity of the rheumatoid factor (RF). Scores for both facets were higher in patients with positivity for RF than in those with a positivity for APF or seronegativity for RF indicating that patients with a positive RF had a better psychological health, more self-esteem, and less negative feelings.

For the sarcoidosis patients, no relationships were found berween physiological measures, that is $\mathrm{SACE}$ levels as well as pulmonary function tests, and QOL.

\section{Discussion}

Generally, parameters of disease activity or progression in RA are measured by the disease activity score (DAS). The DAS has shortcomings with respect to the subjective feeling the RA patient has. In this study, we compared quality of life 
(QOL) in RA with QOL in sarcoidosis using the WHOQOL-100. The results showed that compared with healthy control subjects, QOL was impaired in both RA patients and sarcoidosis patients in many aspects of daily life. Compared to sarcoidosis patients, the impairment of QOL in RA patients was more severe and included more aspects of daily life.

Both patient groups felt anergic and suffered from fatigue. Recurrent or persistent fatigue is a feature of many acute or chronic inflammatory conditions. Fatigue is generally recognized as a subjective feature in RA not being in clinical remission [26]. CROSBY found in a patient group with RA that more than $50 \%$ of patients suffered from fatigue and constant lack of energy [27]. Furthermore, in another study she demonstrated a correlation of pain, fragmented sleep, and functional ability with fatigue in RA patients [28]. In the present study, pain and fatigue were interrelated in the RA patients. However, no interrelationships of pain and fatigue with sleep were found, although all three aspects were significantly impaired. In sarcoidosis, the symptom fatigue has received little attention in literature [29]. Recently, we found lower levels of energy and more fatigue in a group of sarcoidosis patients compared to healthy controls. This finding was not associated with current symptoms; it appeared to be present also in patients who initially had reported no symptoms [8]. In another study, we found besides reduced respiratory muscle strength and respiratory muscle endurance, an interrelationship between the symptom fatigue and decreased expiratory muscle strength [30].

RA can have a negative effect on affective, behavioral, and social functioning of patients [31]. Studying psychological stressors in RA, others have found, that patients with RA have to cope simultaneously with pain, limitations and dependence and that all three factors were negatively related to QOL [32]. Moreover, depressive symptoms are more frequent in patients with RA than in healthy controls, even after controlling for criterion contamination $[31,33]$. In the present study, the RA patients as a group were not impaired with respect to their psychological health. However, patients with lower levels of energy 
showed more impairment of their psychological health. In rheumatology research, the negative affect component of QOL has been emphasized [31]. In the present study, RA patients did not have more negative feelings than sarcoidosis patients and healthy controls. However, patients with seropositivity for rheumatoid factor (RF) showed a better psychological health, less negative feelings, and a better self-esteem compared with positivity for APF. Fewer psychopathological tendencies in patients with positivity for RF than in those with seronegativity for RF have been reported before [34].

Also the sarcoidosis patients included in this study were not impaired in their psychological health. We found no relationship between psychological health and fatigue. With respect to depressive symptoms in sarcoidosis patients, we found in a previous study that patients with current symptoms experienced more depressive symptoms than asymptomatic patients [7].

In the present study, the QOL in both patient groups was impaired mainly with respect to aspects of physical health and level of independence, as well as general health and overall QOL. As discussed above, with respect to fatigue and sleeping problems, the two patient groups did not differ significantly from each other. Also difficulties performing activities of daily living and work were equally impaired in both patient groups. In addition, the RA patients were impaired in their mobility. Also pain and discomfort appeared to be a major problem only in RA patients. This could be expected, as chronic and acute pain is known to be the major consequence of this disorder [31,32]. Surprisingly, no rellationship was found between the levels of pain of RA patients assessed by the WHOQOL-100 and any of the physiological measures of disease severity. In contrast, MEENAN et al. found for the AIMS pain scale relationships with criteria of disease severity such as joint count [35]. However, the AIMS is a health status measure and the questions are closer related to objective measures than the WHOQOL-100 items. The questions of the latter assess, e.g., to what extent the patient has "difficulties to cope with pain or discomfort", "worries about pain or discomfort", and measure the "extent that pain keeps you from doing 
what you must do". These questions assess rather the patient's subjective perception. The impairment the patient perceives by the pain is not necessarily related to measures such as functional status or swollen joint count.

RA patients with elevated CRP levels had low levels of energy and more fatigue. This is in line with other studies, in which improvement of fatigue coincided with a descend of CRP levels after treatment [36]. Previously, we found a relationship between fatigue and CRP levels for sarcoidosis patients as well [37]. Moreover, we found that in sarcoidosis patients, the symptom fatigue is assessed more accurately by the WHOQOL-100 than by only asking patients, whether they have complaints [8]. The WHOQOL-100 facet energy and fatigue appears to be sensitive for the assessment of fatigue in RA patients as well.

Physiological measures such as ESR and the current medication as well as medication in the past were related to only a few aspects of QOL. As discussed above, elevated CRP levels correlated with patients' pain and discomfort. No relationship was found between duration of the disease and QOL. In general, non-significant correlations were found between QOL and the routinely performed physiological measures. These results are in line with other studies that have shown that disease status in RA, assessed by the routinely performed measures of physical health, did not reflect the impact of RA on psychosocial well-being [38]. Self-reported low physical well-being, however, was associated with more depressive feelings [6]. Other subjective stressors such as perceived limitation and dependence were found only weakly related to traditionally used disease status measures [32].

For the sarcoidosis patients no relationship between pulmonary function tests such as FEV, and T,, co and the WHOQOL-100 scores were found. Also health status was not related to duration of the disease and routinely performed tests such as pulmonary function tests and serum angiotensin converting enzyme (sACE) levels, respectively, as we showed in a previous study [7]. However, as mentioned above, fatigue was found related to higher levels of CRP in sarcoidosis patients [37]. Although the impairment of QOL was more 
severe in the RA patients, QOL was considerably impaired in the sarcoidosis patients as well. When only considering the pulmonary function tests, one would expect only mild impairment of QOL in these sarcoidosis patients, as these tests were normal in most patients, or showed only mild impairment.

The present study has a couple of limitations. Disease severity as assessed by the different, routinely performed physiological measures for both disorders are not directly comparable. Moreover, the disease duration of the RA patients was longer than that of the sarcoidosis patients. Although one may hypothesize that duration of the disease influences QOL, this was clearly not the case in both patient groups. In addition, the RA patients were significantly older than the sarcoidosis patients. This difference between the patient groups did not influence the results, as age was used as covariate in the analyses.

In conclusion, QOL is impaired in both RA as well as in sarcoidosis. As hypothesized, QOL was more seriously impaired and included more aspects of QOL in RA patients than in sarcoidosis patients. Main problems for both patient groups were sleeping problems and fatigue. As expected, pain and impaired mobility were found only in the RA patients. However, in contrast to expectations, with respect to their working capacity and problems performing activities of daily living the two patient groups did not show any differences. Although the disease impact on the quality of life was more severe in the RA patients, the impact of the disease on symptomatic sarcoidosis patients was considerable. This study indicated that QOL indeed is partly related to physiological measures of disease severity in RA, but not at all in sarcoidosis. Thus, in RA, as in other chronic diseases such as sarcoidosis, assessment of QOL is of additional value and, therefore, should be included in the follow-up for a broad evaluation of the patients' clinical situation. The WHOQOL-100 appeared to be an appropriate instrument to assess QOL in both diseases studied. 


\section{Acknowledgements}

The authors thank the Departments of Pulmonology of the participating Dutch Hospitals and the Department of Rheumatology of the Rijnstate Hospital, Arnhem. Moreover, we would like to thank S. Drent, L.M.M. Kock, M. Janssen, A.J.L. de Jong, and N. de Vries for their help in collecting the data, and M.D.P. Elfferich for her valuable advice and comments.

\section{References}

1. Patrick DL, Deyo RA. Generic and disease-specific measures in assessing health status and quality of life. Med Care 1989;27: S217-\$232.

2. Blalock SJ, McEvoy deVellis B, deVellis RF, Sauter van SH. Self-evaluation processes and adjustment to rheumatoid arthritis. Arthritis Rheum 1988; 31: 1245-1251.

3. Long AF, Scott DL. Meeting Report. Measuring health status and outcomes in rheumatoid arthritis within routine clinical practice. Br J Rheumatol 1994; 33: 682-685.

4. Van Riel P, Van Lankveld W. Quality of life in rheurnatoid arthritis: a review. Pharm World Sci 1993; 15:93-97.

5. Meenan RF. The AIMS approach to health status measurement: conceptual background and measurement properties. J Rheumatol 1982; 9: 785-788.

6. Bijlsma JWJ, Huiskes CJAE, Kraaimaat FW, Vanderveen MJ, Huber-Bruning $O$. Relation between patients' own health assessment and clinical and laboratory findings in rheumatoid arthritis. $J$ Rheumarol 1991; 18:650-653.

7. Drent M, Wirnsberger RM, Breteler MHM, Kock LMM, De Vries J, Wouters EFM. Quality of life and depressive symptoms in patients suffering from sarcoidosis. Sarcoidosis Vasc Diffuse Lung Dis 1998; 15: 59 -66.

8. Wirnsberger RM, De Vries J, Breteler MHM, Van Heck GL, Wouters EFM, Drent M. Evaluation of quality of life in sarcoidosis patients. Respir Med 1998; 92: 750756.

9. Semenzato $G$, Zambello R, Trentin L, Agostini C. Cellular immunity in sarcoidosis and hypersensitivity pneumonitis. Recent advances. Chest 1993; 103 (suppl): 139S-143S.

10. Bennett JC. The etiology of Rheumatoid arthritis. In: Kelley WN, Harris ED, Ruddy S, Sledge CB, eds. Textbook of Rheumatology. Philadelphia, Saunders, 1981: $887-895$.

11. King TE. Connective tissue disease. In: Schwarz MI, King TE, eds. Interstitial Lung Disease. St. Louis, Missouri; Mosby-Year Book, 1993: 271-308.

12. Rees JH, Woodhead MA, Sheppard MN, duBois RM. Rheumatoid arthritis and crypogenic organizing pneumonia. Respir Med 1991; 85: 243-246. 
13. Wirnsberger RM, De Vries J, Wouters EFM, Drent M. Clinical presentation of sarcoidosis in the Netherlands. An epidemiological study. Neth J Med 1998; 53: 53-60

14. Ruta DA, Hurst NP, Kind P, Hunter M, Stubbings A. Measuring health status in British patients with rheumatoid arthritis: reliability, validity and responsiveness of the Short Form 36-item Health Survey (SF-36). BrJRheumatol 1998; 37:425436.

15. Fries JF, Spitz P, Kraines RG, Holman HR. Measurement of patient outcome in arthritis. Arthritis Rheum 1980;23:137-145.

16. Fries JF, Spitz PW, Young DY. The dimensions of health outcomes: The Health Assessment Questionnaire, disability and pain scales. I Rheumat 1982; 9: 789793.

17. Pincus T, Summey JA, Soraci SA, Wallston KA, Hummon NP. Assessment of patient satisfaction in activities of daily living using a modified Stanford Health Assessment Questionnaire. Arthritis Rhewm 1983; 26: 1346-1353.

18. O'Boyle CA. The schedule for the evaluation of individual quality of life (SEIQoL). Int $J$ Ment Health 1994; 23: 3-23.

19. De Vries J, Van Heck GL. Nederlandse WHOQOL-100, 1995. Tilburg: Tilburg University.

20. Arnett FC, Edworthy SM, Bloch DA, et al. The American Rheumatism Association 1987 revised criteria for the classification of rheumatoid arthritis. Arthritis Rheum 1988; 31:315-324.

21. Steinbrocker $\mathrm{O}$, Traeger $\mathrm{CH}$, Batterman RC. Therapeutic criteria in rheumatoid arthritis. JAMA 1949; 140:659-662.

22. WHOQOL group. Development of the WHOQOL: rationale and current status. Int J Ment Health 1994; 23: 24-56.

23. De Vries J. Beyond health status. Construction and validation of the Dutch WHO Quality of life assessment instrument. Doctoral thesis, Tilburg University, Tilburg, The Netherlands, 1996.

24. Quanjer PH, Tammeling GJ, Cotes JE, Pedersen OF, Peslin R, Yernault J-C. Lung volumes and forced ventilatory flows. Report working party. Standardization of lung function tests. European Commission for Steel and Coal. Official statement of the European Respiratory Society. Eur Respir J 1993; 6 (suppl 16): 5-40.

25. SPSS-PC for the IBM PC/XT/AT. SPSS Inc., Chicago, 1986.

26. Pinals RS, Masi AT, Larsen RA. Preliminary criteria for clinical remission in rheumatoid arthritis. Arthritis Rheum 1981;24: 1308-1315.

27. Crosby LJ. Stress factors, emotional stress and rheumatoid arthritis disease activity. $J$ Adv Nurs 1988; 13:452-461.

28. Crosby LJ. Factors which contribute to fatigue associated with rheumatoid arthritis. $J$ Adv Nurs 1991; 16:974-981.

29. James DG. Complications of sarcoidosis. Chronic fatigue syndrome. Sarcoidosis $1993 ; 10: 1-3$.

30. Wirnsberger RM, Drent $M$, Hekelaar $N$, et al. Relationship between respiratory muscle function and quality of life in sarcoidosis. Eur Respir $J$ 1997; 10: 14501455. 
31. Anderson KO, Bradley LA, Young LD, McDaniel LK, Wise CM. Rheumatoid arthritis: review of psychological factors related to etiology, effects, and treatment. Psychol Bull 1985; 98: 358-387.

32. Van Lankveld W, Näring G, Van der Staak C, Van 't Pad Bosch P, Van de Putte $L$. Stress caused by rheumatoid arthritis: the relation between subjective stressors of the disease, disease status and well-being. $J$ Behavioral Med 1993; 16:309. 321 .

33. Pincus $T$, Callahan LF. Depression scales in rheumatoid arthritis: criterion contamination in interpretation of patient responses. Patient Educ Couns 1993; 20: 133-143.

34. Vollhardt BR, Ackerman SH, Grayzel AI, Barland P. Psychologically distinguishable groups of rheumatoid arthritis patients: a controlled, single blind study. Psychosom Med 1982; 44: 353-362.

35. Meenan RF, Gertman PM, Mason JH, Dunaif R. The Arthritis Impact Measurement Scales: further investigations of a health status measure. Arthritis Rheum 1982;25: 1048-1053.

36. Elliott MJ, Maini RN, Feldmann M, et al. Randomised double-blind comparison of chimeric monoclonal antibody to tumour necrosis factor $\alpha$ (cA2) versus placebo in rheumatoid arthritis. Lancet 1994; 344: 1105-1110.

37. Drent M, Schols AMWJ, Wouters EFM. Alterations in metabolism and body composition associated with an acute phase response in patients suffering from sarcoidosis. Sarcoidosis Vasc Diffuse Lung Dis 1997; 14 (suppl 1): 43.

38. Van Lankveld WGJM. Coping with chronic stressors of rheumatoid arthritis Doctoral thesis, Catholic University Nijmegen, Nijmegen, The Netherlands, 1993. 
Chapter 7

Relationship between respiratory muscle function and quality of life in sarcoidosis 


\section{Relationship between respiratory muscle function and quality of life in sarcoidosis}

R.M. Wirnsberger*, M. Drent*, N. Hekelaar**, M.H.M. Breteler***, S. Drent***, E.F.M. Wouters*, P.N.R. Dekhuijzen**

*Dept of Pulmonology, University Hospital Maastricht, ${ }^{*}$ Dept of Pulmonology, Academic Hospital Nijmegen, ***Dept of Clinical Psychology and Personality, University Nijmegen, The Netherlands

Eur Respir J 1997; 10: 1450-1455 


\begin{abstract}
In sarcoidosis, pulmonary and general symptoms often do not correlate with radiographic stage and routinely performed lung function tests. Asymptomatic muscle involvement in sarcoidosis is common, but little is known about respiratory muscle involvement. The aim of this study was to investigate any relationships between persistent complaints and/or quality of life and respiratory muscle strength and endurance, respectively.

Measurements of maximal inspiratory and expiratory mouth pressures $\left(P_{1, \max }\right.$ and $\left.P_{\mathbb{E}, \max }\right)$, respiratory muscle endurance and routine lung function were made in 18 patients with sarcoidosis. To assess health status and quality of life, patients completed the Sickness Impact Profile (SIP).

Respiratory muscle strength and endurance time were lower in the patient. group than in a group of healthy controls $(p=0.05)$. Compared to a general population, the patients with sarcoidosis were found to be limited in physical and psychosocial functioning. The respiratory muscle endurance time correlated with the SIP subscalles "mobility" $(r=-0.56 ; \mathrm{p}<0.01)$, and "body care and movement' $(x=-0.79 ; p<0.001)$. The total lung capacity (TLC), inspiratory vital capacity (IVC) and forced expiratory volume in one second $\left(\mathrm{FEV}_{1}\right)$ were normal in all subjects.

In conclusion, patients with sarcoidosis and normal lung function showed reduced respiratory muscle strength and endurance time. Correlations were found between these indices and both symptoms and certain Sickness Impact Profile domains. Therefore, we suggest inclusion of measurements of respuratory muscle strength in the assessment and follow-up of patients with sarcoidosis.
\end{abstract}




\section{Introduction}

Sarcoidosis is a multisystem disorder, which can affect essentially every organ of the body [1]. The parts of the body most frequently involved are the lungs, lymph nodes, skin, eyes, muscles, heart and joints, and, therefore, symptoms vary considerably. In sarcoidosis, disease activity does not necessarily indicate a progressive or fatal course [2]. Even though the active disease often subsides spontaneously, an indication of activity is necessary in order to monitor the course of the disease and to guide treatment, should the clinical situation justify it $[2,3]$.

Whilst there is no single test that accurately reflects the progression of sarcoidosis, clinical examination, chest radiography and lung function tests are used to evaluate and monitor the disease process. Techniques useful in assessing the extent and severity of granulomatous inflammation and fibrosis are: 1) serum markers, including serum angiotensin converting enzyme (sACE); 2) ${ }^{67} \mathrm{Ga}$ scan; 3) bronchoalveolar lavage (BAL); and 4) high resolution computed tomography (HRCT) $[2,4]$. When indicators of disease activity, such as radiographic abnormalities, lung function impairment and $\mathrm{SACE}$, return to normal, non specific symptoms, such as fatigue and reduced exercise tolerance, may persist.

In a previous study, we found that the quality of life measured by the Sickness Impact Profile (SIP) was related to the perception of complaints, but not to disease activity as conventionally assessed [5]. The SIP is a generic health status instrument, which is intended to measure the impact of sickness rather than to objectively measure the presence of disease [6]. It is an individual's own experience of illness perceived through its effect on daily activities, feelings and attitudes [7].

Asymptomatic granulomatous muscle involvement in sarcoidosis has been reported with a prevalence of $50-80 \%$ [8], whereas symptomatic muscle 
involvement is much less common (range 1.4-2.3\%)[9]. The symptomatic involvement that has been described varies from a palpable nodular type to an acute myositis, and a chronic myopathic type [9-12]. Usually, patients present with pain, weakness and muscle atrophy [13]. Little is known, however, about the frequency of respiratory muscle involvement [14].

The aim of this study was to investigate whether there is a relationship between persistent complaints or quality of life disturbance and respiratory muscle function in a population of patients with sarcoidosis. Therefore, the relationships between respiratory muscle strength, endurance time, and the SIP scores and subscores were evaluated.

\section{Materials and methods}

\section{Subjects}

Eighteen out-patients suffering from sarcoidosis, who attented the Academic Hospital Nijmegen, were studied. The diagnosis of sarcoidosis was based on consistent clinical. features, together with biopsy-proven noncaseating epitheloid cell granuloma. The clinical symptoms of the respective patients varied from none ( sarcoidosis detected on routine chest radiography) to more or less severe respiratory symptoms or erythema nodosum and arthralgia (i.e. Löfgren's syndrome). None of the participating subjects had any significant medical history that might have influenced quality of life. Patients with significant co-morbidity were excluded.

A control group $(n=18)$, comprising heal thy employees of the Academic Hospital Nijmegen matched for age and gender, were used as reference for the lung function tests, respiratory muscle strength and endurance time, as well as peripheral muscle strength. A control group comprising 594 subjects from a general Dutch population (mean age 43 yrs, range $18-75$ yrs; 267 males ( $45 \%$ ) and 327 females (55\%) were used as reference to compare the SIP measurements [15]. 


\section{Chapter 7}

\section{Study design}

Initially, 26 patients with sarcoidosis were contacted. Eight declined for various personal reasons. Eighteen patients agreed to participate after giving informed consent. The questionnaires were completed under supervision of a study assistant, to avoid missing data or misunderstanding. In addition, the patients were asked about their smoking history and whether they had any of the following complaints: fatigue, dyspnea, cough, arthralgia, or erythema nodosum.

At the time the study was performed, seven of the patients still had symptoms, most notably fatigue. Eleven of the 18 patients had not taken corticosteroids during the previous 3 months. The other seven patients were taking corticosteroids during the 3 months prior to the study, with an average prednisone dose of $5.7 \mathrm{mg} \cdot \mathrm{day}^{-1}$. Five of these seven patients still had symptoms. The mean ( \pm SD) SACE level was $16.8 \pm 5.9$ $\mathrm{U} \cdot \mathrm{L}^{-1}$ ( reference value $<20 \mathrm{U} \cdot \mathrm{L}^{-1}$ ). The radiographic stage varied from stage 0 to III: four patients had stage 0 , two stage I, 10 stage II, and two stage III appearances. Demographic and clinical characteristics of the patients and the control group are summarized in table 1.

Table I. - Characteristics of patients with sarcoidosis and control subjects

Variable

Sarcoidosis patients

Control subjects

$(n=18)$ $(n=18)$

Age yrs

$43(11)$

$41(11)$

Gender male/fernale

Smoking yes/no

Complaints yes/no

Time since diagnosis yrs

$7.2(7.1)$

Data are expressed as absolute number or mean, and SD in parenthesis. 


\section{Lung function tests}

Lung function measurements included forced expiratory volume in one second ( $\mathrm{FEV}_{1}$ ), inspiratory vital capacity (IVC) measured with a pneumotachograph, and total lung capacity (TLC) measured using a body plethysmograph (Compactbody; Jaeger, Würzburg, Germany). The best of three efforts was selected. All volumes were expressed as percentages of the reference values [16].

The transfer factor of the hung for carbon monoxide $(T L, C O)$ was measured by the single-breath method (Masterlab; Jaeger, Würzburg, Germany). In order to compare the $T L$, co level with the quality of life scores, the values were classified in four ranges: 1) normal $(\geq 80 \%)$; 2) mild decrease ( $60-80 \%)$; 3) moderate decrease (40-60\%); and 4) severe decrease $(<40 \%$ of predicted), according to the American Medical Association classes.

\section{Respiratory muscle strength and respiratory muscle endurance}

Inspiratory and expiratory muscle strength were assessed by measuring maximal respiratory mouth pressures using the method of BLACK and HYATT [17]. Maximal inspiratory mouth pressure $\left(P_{1}, \max \right)$ was measured at residual volume (RV), whilst maximal expiratory mouth pressure $\left(P_{\mathrm{E}, \mathrm{max}}\right)$ was measured at total lung capacity (TLC). The equipment used was a pressure transducer, (model MP 45-30; Validyne Engineering Corp., Northridge CA, USA). All signals were recorded on a strip chart, (type BD 31; Kipp \& Zonen, Delft, The Netherlands). A needle was placed in the proximal end of the mouthpiece to ensure that efforts were performed with open glottis. At least five manoeuvres were performed, until the three highest values were within $5 \%$ of each other. All subjects were seated in the upright position and were wearing a noseclip while performing the tests, and the same instructions were given to each subject by one investigator. Values are expressed in absolute terms and as percentages of predicted values according to Whison et al. [18].

Respiratory muscle endurance was assessed by measuring the endurance time using a modiffed threshold loading device as designed by NickERSON and KEENS [19]. This device has an inspiratory valve, which can be occluded by a weighted plunger. The 


\section{Chapter 7}

plunger can be adjusted externally so that patients must generate enough inspiratory pressure to lift the inspiratory valve and allow inspiratory flow. Expiration can be performed without any resistance. The threshold loading device was first tested and validated in healthy subjects before use. There was a very close relationship between the inspiratory pressure $(\mathrm{PI})$ generated and external weight $(\mathrm{W})(\mathrm{Pr}=-(0.201126 \mathrm{xW}+7.4659)$; $r=0.999, p<0.001 ; \mathrm{Pl}_{1}$ in $\mathrm{cmH}_{2} \mathrm{O}, \mathrm{W}$ in $\left.\mathrm{g}\right)$. The respiratory muscle endurance time was defined as the maximal time (in seconds) during which a subject could sustain breathing against an inspiratory pressure load equal to $70 \%$ of his/her individual $P_{1, \max }$ [20]. All subjects first warmed up by breathing against an inspiratory pressure load equal to $15 \%$

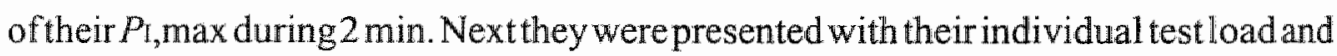
instructed to breathe for as long as they could, until they became so tired they could not continue or until they were unable to get enough air. The test was terminated if the respiratory muscle endurance time exceeded $15 \mathrm{~min}$. During the test the inspiratory pressure was recorded constantly on the strip chart as described.

\section{Peripheral muscle strength}

Quadriceps force was measured on the nondominant side with the subject sitting, both hip and knee in $90^{\circ}$ flexion, the ankle connected to measuring-equipment based on strain gauges. A nonstretching-band was placed around the hip to prevent the subject from lifting up whilst pulling away the lower leg. The force of the hand muscles was also measured on the nondominant side with the subject sitting, the forearm and hand in a direct line with each other and resting on a table, and the fingers enclosing two wooden handles connected to the measuring equipment based on strain gauges. In both tests, at least three isometric contractions were performed until the differences were not more than $5 \%$. Between each effort there was an interval of 1 min. Values are expressed as kilogram force $(\mathrm{kgf})$.

\section{Health status questionnaire}

Health status and quality of life were assessed with the SIP (Dutch version) $[15,21]$. The SIP was designed to assess sickness-related behavioural dysfunction. It provides 
summary scores for physical, psychosocial, and overall behavioural dysfunction, as well as separate scores for 12 categories of activity. The scores are expressed as percentages of the maximal possible score of dysfunction in that particular category or set of categories [22]. The scores range between 0 and 100. The higher the scores, the stronger the impact of the disease on the individual's life.

\section{Statistical analysis}

The data of the control subjects and patients were compared using Student's $t$-tests for binomial values and Chi-squared tests for ordinal values. Differences in personal characteristics were assessed using Chi-squared tests for categorical data and Student's $t$-tests for continuous data. Correlation among variables was assessed by single regression analysis. Because of the large number of correlations examined, a probability value of less than 0.01 was considered to be statistically significant. All analyses were performed using the Statistical Package for Social Science (SPSS).

\section{Results}

Tables II and III summarize the results of the SIP scores, and the lung function tests, respiratory and peripheral muscle strength assessment, respectively.

The total SIP scores for the patient group were significantly higher compared with the control subjects. In the patients with sarcoidosis, the subscores were significantly higher in the subscales 'alertness behaviour', "home management', 'recreation and pastimes', 'sleep and rest', 'social interaction', and 'employment' (table II).

Only one of the 18 patients had a slightly decreased $T$,, $\mathrm{Co}$. The absolute values for $\mathrm{FEV}$, as well as $\mathrm{FEV}, \%$ pred were within the normal range for all patients, as also were the VC and TLC. However, FEV,$\%$ pred was lower in the patients than in the control subjects $(\mathrm{p}<0.05)$. Pl, max, PE, max, and respiratory muscle endurance time were all lower in the sarcoidosis patients 
compared to the control subjects $(p<0.05)$. Only one of the control subjects failed to complete the respiratory muscle endurance test for the $15 \mathrm{~min}$ period, whereas six of the 18 sarcoidosis patients did not finish the test $(\mathrm{p}<0.05)$. No differences were found in the respiratory muscle strength and endurance time between sarcoidosis patients who were treated with prednisone and those who were not.

Peripheral muscle strength, i.e. quadriceps and hand muscle force, showed no differences between the sarcoidosis patients and control subjects (table III).

Table II. - Average Sickness Impact Profile (SIP) scores for the sarcoidosis patients and a general Dutch population

\begin{tabular}{lcc} 
SIP & $\begin{array}{c}\text { Sarcoidosis patients } \\
(\mathrm{n}=18)\end{array}$ & $\begin{array}{c}\text { Control subjects } \\
(\mathrm{n}=594)\end{array}$ \\
\hline Alertness behaviour & $9.9(20.7)^{* *}$ & $4.6(12.4)$ \\
Ambulation & $4.1(8.4)$ & $3.0(7.8)$ \\
Body care and movement & $1.7(3.8)$ & $1.9(5.0)$ \\
Communication & $2.5(4.3)$ & $1.1(4.5)$ \\
Eating & $1.1(3.4)$ & $1.0(3.2)$ \\
Emotional behaviour & $5.7(12.3)$ & $4.0(10.0)$ \\
Home management & $6.9(11.3)^{* *}$ & $4.8(11.8)$ \\
Mobility & $2.9(5.6)$ & $2.4(7.6)$ \\
Recreation and pastimes & $0.5(15.8)^{* *}$ & $7.6(14.1)$ \\
Sleep and rest & $14.3(16.9)^{* *}$ & $4.8(8.2)$ \\
Social interaction & $14.0(23.5)^{* *}$ & $4.0(8.4)$ \\
Employment & $12.4(18.8)^{* *}$ & $7.3(20.0)$ \\
Physical dimension & $2.6(4.4)$ & $2.2(5.4)$ \\
Psychosocial dimension & $11.7(18.6)^{* *}$ & $3.5(7.2)$ \\
Total SIP score & $8.0(9.7)^{* *}$ & $3.4(5.6)$ \\
& & \\
\hline
\end{tabular}

Data are expressed as mean, and sD in parentheses. The score are on a scale ranging 0 100. ${ }^{* *} \mathrm{p} \leq 0.01$ sarcoidosis patients $v$. controls (Student's $t$-test). 
Table III. - Lung, respiratory and peripheral muscle function of patients and control subjects

Sarcoidosis patients

$$
(\mathrm{n}=18)
$$

Control subject

$$
(n=18)
$$

IVC \% pred

FEV, L

FEV $\%$ pred

$T \mathrm{~L}, \mathrm{CO} \%$ pred

TL,co normal/decreased

$P_{1, \max }$ (at RV)

PE,max (at TLC)

Respiratory muscle

Endurance test completed $\mathrm{n}$

Endurance time s

Quadriceps force kgf

Male

Female

Hand muscle force $\mathrm{kgf}$

Male

Female

$99(11)$

$3.1 \quad(0.9)$

$92(10)^{* *}$

$86 \quad$ (9)

$17 / 1$

$-86.8(28.7)^{* * *}$

$96(27)^{* *}$

$91.6(31.5)^{* *}$

$74(19)^{* *}$

12

$756(246)^{* *}$

$43.1 \quad(14.1)$

$25.6(8.4)$

$48.5(12.8)$

$23.1(6.8)$
105

$3.6(0.7)$

106 (12)

$100 \quad(8)$

$18 / 0$

$-112.8(24.8)$

$130 \quad(28)$

$126.1(34.8)$

107 (20)

$869 \quad(130)$

$45.4(9.1)$

$32.5(4.8)$

$52.1(8.6)$

$33.5(5.4)$

Data are expressed as absolute number or mean and SD in parenthesis. IVC: inspiratory vital capacity; \% pred: percentage of predicted value; $\mathrm{FEV}_{1}$; forced expiratory volume in one second; $T \mathrm{~L}, \mathrm{Co}$ : tranfer factor of the lung for carbon monoxide; $P_{\mathrm{I}}$, max: maximal inspiratory mouth pressure; $\mathrm{RV}$ : residual volume; $P_{\mathrm{E}, \mathrm{max}}$ : maximal expiratory mouth pressure; TLC: total lung capacity. ${ }^{* *} \mathrm{p} \leq 0.01$ sarcoidosis patients $v s$. controls (Student's $t$-test).

The respiratory muscle endurance time correlated with the SIP subscales 'mobility' $(p<0.01)$ and 'body care and movement' $(p<0.001)$. However, no correlations were found between the SIP scores and Pr,max, PE, max and peripheral muscle strength, respectively (table IV). Patients with symptoms 
$(\mathrm{n}=7$, table $\mathrm{I})$ showed lower $P_{1, \max }(\mathrm{p}<0.03)$ and respiratory muscle endurance time $(p<0.005)$, while patients who experienced fatigue $(n=6)$ had a lower $P$ E, $\max (p<0.03)$ compared to those without complaints.

Table IV. - Univariate regression analyses between Sickness Impact Profile (SIP) scores of patients with sarcoidosis and respiratory muscle strength, endurance time and peripheral muscle strength

\begin{tabular}{lccccc}
\hline SIP & $P_{\mathrm{I}, \max }$ & $P_{\mathrm{E}, \max }$ & $\begin{array}{c}\text { Endurance } \\
\text { time }\end{array}$ & HMF & $\mathrm{QF}$ \\
\hline Mobility & & & & & \\
r & -0.02 & -0.28 & -0.56 & 0.12 & 0.10 \\
p-value & $\mathrm{NS}$ & $\mathrm{NS}$ & $<0.01$ & $\mathrm{NS}$ & $\mathrm{NS}$ \\
$\begin{array}{l}\text { Bodly care and movement } \\
\text { r }\end{array}$ & -0.30 & -0.07 & -0.79 & 0.17 & 0.22 \\
p-value & $\mathrm{NS}$ & $\mathrm{NS}$ & $<0.001$ & $\mathrm{NS}$ & $\mathrm{NS}$ \\
\hline
\end{tabular}

HMF: hand muscle force; QF: quadriceps force; NS: not significant. For further definitions see legend to table III.

The radiographic stage was related to the SIP subscales 'alertness behaviour' ( $p<0.001)$, 'emotional behaviour' $(p<0.001)$, 'home management' $(p<0.005)$ and 'social interaction' ( $p<0.001$ ). The duration of disease, the FEV $S A C E$ levels showed no relationships with percentile scores of any of the subscales. Furthermore, no relationships between radiographic stage, sACE, respiratory muscle strength, and respiratory muscle endurance time were found.

\section{Discussion}

This study has shown that respiratory muscle strength and respiratory muscle endurance are reduced in patients with sarcoidosis. Patients with the symptom 
of fatigue had significantly lower maximum expiratory pressures than those without symptoms. A decreased respiratory muscle endurance time suggests the presence of respiratory muscle fatigue. Muscle fatigue has been defined as a condition in which there is a reduction in the capacity for developing force and/or velocity of a muscle in response to a load, and is reversible by rest [23]. In general, muscle fatigue occurs whenever energy demand exceeds energy supply [24]. Applying this to respiratory muscles, fatigue occurs when these muscles work under conditions where there is an imbalance between energy demand and supply [24]. Muscle weakness is a condition in which the capacity for a rested muscle to generate force is decreased [23]. In our patient group, we found decreased $P_{\mathrm{I}}$, max and $P_{\mathrm{E}, \mathrm{max}}$, which indicates inspiratory as well as expiratory muscle weakness. Previously, reduced respiratory muscle strength in sarcoidosis was reported by BAYDUR et al. [13], who compared untreated patients suffering from sarcoidosis with control subjects, and found a decrease in maximal mouth pressures $\left(P_{1}, \max \right)$ in the sarcoidosis patients. In agreement with this, BADR and SHARMA [14] found respiratory muscle dysfunction in $61 \%$ of a group of sarcoidosis patients. Moreover, in their study, the $P_{E}$, max was significantly lower than in normal controls [14].

The results of the present and previous studies, therefore, suggest that $P_{\mathrm{I}, \text { max }}$ and $P \mathrm{E}, \mathrm{max}$ are related to functional impairment due to sarcoidosis. These results also emphasize the need for an integrative approach in the assessment and therapeutic management of sarcoidosis. It should be noted, however, that the respiratory muscle tests applied in the present study depend on the cooperation of the patient. To the best of our knowledge, nonvolitional tests of respiratory muscle function, such as magnetic or electrical stimulation of the phrenic nerves, have not been reported in these patients.

Inspiratory muscles are the major muscle groups used in supporting the ventilatory demands of exercise, and the effort required to drive the inspiratory muscles during exercise is a major contributor to the sensation of exertional dyspnea [25]. With weak muscles, greater utilization of the available muscle 
mass is required to maintain a given external power output, resulting in an increased sense of effort. As a consequence, in persons with weak respiratory muscles, intolerable exertional discomfort and exertional dyspnea occur at lower power outputs than those with strong muscles [25]. In the present study, patients with symptoms had a decreased inspiratory muscle strength and endurance time, when compared to those without symptoms. The complaint of 'fatigue' was associated with a lower expiratory muscle strength. Moreover, a relationship was found between the $P$, max and respiratory muscle endurance time. Thus, reduced respiratory muscle strength in the presence of sarcoidosis may contribute to exercise intolerance independent of impairment in ventilatory function. This conclusion is consistent with that of HAMIL TON et al. [25], who showed that in patients with cardiorespiratory disorders, respiratory, as well as peripheral, muscle strength was a significant contributor to symptom intensity and work capacity, in addition to any contribution from ventilatory, gas exchange, and circulatory impairment.

Several factors, such as hypoxaemia, hypercapnia, malnutrition, decreased blood supply to the muscles, drugs, and electrolyte abnormalities, contribute to respiratory muscle weakness and fatigue, e.g. in chronic obstructive pulmonary disease patients [26, 27]; however, these are unlikely to contribute in sarcoidosis. Which factor or combination of factors is responsible for this effect in sarcoidosis has not yet been established. In the absence of metabolic abnormalities, recent data have emphasized the role of inflammatory products on different aspects of muscle metabolism. As in other immune-mediated disorders, most clinical manifestations of sarcoidosis are secondary to the direct effect of the accumulation of activated immunocompetent cells in the involved tissues, notably helper $\mathrm{T}$ lymphocytes and macrophages, and by an increased production of different: cytokines $[28,29]$. These cytokines appear to be produced in inflamed lesions of sarcoidosis and released into the bloodstream [30]. Future identification of these products could lead to specific therapeutic interventions aimed at blocking their effect in the clinical management of patients with inflammatory disorders, 
such as sarcoidosis.

As previously suggested, impaired respiratory muscle function suggests that the respiratory muscles can be involved in the granulomatous process of sarcoidosis [13]. Many reports have described granulomatous myopathy without overt manifestations of sarcoidosis elsewhere; sometimes a past history of sarcoidosis was suggested. Hence, granulomas in muscles may persist after those in other organs have become inactive or have resolved in patients who have passed through the active generalized stages of sarcoidosis without obvious symptoms [31]. Little is known about involvement of respiratory muscles, as only a few cases have been described $[14,32,33]$. For example, it is not known whether diaphragm and/or intercostal muscle involvement in sarcoidosis is a possible contributory factor to dyspnea or impairment of vital capacity [31].

However, histological confirmation of respiratory muscle involvement is hard to obtain. Furthermore, myopathy, including disorders of the respiratory muscles, is a well-known sideeffect of corticosteroids [34, 35]. It should be considered especially with higher doses of steroids, in the presence of markedly impaired respiratory muscle strength or continuous treatment with fluorinated steroids [36]. In the present group of patients, only seven had been treated with steroids during the 3 months prior to the study and the doses they received were low. Moreover, the respiratory and peripheral muscle strength did not differ between patients with or without a history of corticosteroid treatment.

The $T \mathrm{~L}, \mathrm{CO}$ is considered by some authors to be the most sensitive index of lung function in sarcoidosis. It is often the first or only index to be decreased [14]. Remarkably, however, in the present study the respiratory muscle force and endurance were significantly lower than in the control group, even though other lung function tests were normal. This suggests that maximal mouth pressure and respiratory muscle endurance time may be more sensitive to functional impairment due to sarcoidosis than are the $T \mathrm{~L}, \mathrm{co}$ or $\mathrm{VC}$, which have been suggested as the best indices to correlate with working capacity [37]. The 
relationship between radiographic stage, lung function and exercise abnormality have, however, been variable [38]. Clearly, there is a wide spectrum of tissue inflammation and organ dysfunction within, as well between, each radiographic stage. KARETZKY and MCDONOUGH [38] found that the magnitude of functional impairment may vary widely from the apparent histopathologic involvement as reflected by chest radiography and lung volumes. In the present study, we found a relationship between the radiographic stage and impairment in the fields 'alertness behaviour', 'emotional behaviour', 'home management' and 'social interaction'.

Furthermore, in the present study, the SIP subscales 'mobility" and 'body care and movement' as well as the presence of symptoms were shown to correlate with respiratory muscle endurance time. However, for both of these latter domains there was no difference between patients and the control subjects. Moreover, our results show that patients with sarcoidosis are limited in physical and psychosocial functioning. They appear to be particularly affected in the fields 'sleep and rest', 'recreation and pastime', 'employment', 'alertness behaviour', 'home management' and 'social interaction', compared to a control group [15]. This diversity illustrates the broad impact of sarcoidosis on patients quality of life.

In conclusion, respiratory muscle strength and endurance are reduced in patients with sarcoidosis. Decreased respiratory muscle endurance was found to be related particularly to the Sickness Impact Profile subscales 'mobility' and 'bodycare and movement'. However, no such relationship was found between these latter Sickness Impact Profile subscales and routinely performed lung function tests, such as transfer factor and spirometry. Measuring respiratory muscle function seems to quantify and characterize the functional impairment in patients with sarcoidosis and reflects symptoms, such as fatigue and general weakness, that are otherwise difficult to assess objectively. Therefore, we suggest that measurements of respiratory muscle strength and endurance time could be usefully included in the diagnostic work-up and 
follow-up of patients with sarcoidosis. Further studies are needed to assess respiratory and peripheral muscle involvement, as well as the relationship with the presence of inflammatory characteristics in sarcoidosis.

\section{Acknowledgements}

The authors would like to thank H. M. Jacobs for providing the Dutch SIP control data, M.D.P. Elfferich and L.M.M. Kock for their great help in collecting the data and their advice. Furthermore, they would like to thank Ch. van der Grinten and G. Vissers for their technical advice, J. de Vries for statistical assistance, and the lung function laboratory workers, in particular L. van de Pol, for technical assistance and advice.

\section{References}

1. Thomas PD, Hunninghake GW. Current concepts of the pathogenesis of sarcoidosis. Am Rev Respir Dis 1987; 135: 747-760.

2. Sharma OP, Alam S. Diagnosis, pathogenesis and treatment of sarcoidosis. Curr Opin in Pulm Med 1995; 1: 392-400.

3. Hunninghake GW, Gilbert S, Pueringer R, et al. Outcome of the treatment of sarcoidosis. Am J Respir Crit Care Med 1994; 149: 893-898.

4. Baudouin SV, du Bois RM. Disease activity. In: James DG, ed. Sarcoidosis and Other Granulomatous Disorders. New York, Dekker, 1994; 153-180.

5. Drent M, Breteler MHM, Kock LMM, Wouters EFM. Quality of Life (QOL) and depressive symptoms in patients with sarcoidosis: a pilot study. Am J Respir Crit Care Med 1996; 153: A279.

6. Bergner M, Bobitt RA, Carter WB, Gilson BS. The Sickness Impact Profile: development and final revision of a health status measure. Med Care $1981 ; 19$ : 787-805.

7. Davies GL, Balart LA, SchiffER, et al. Assessing health-related quality of life in chronic hepatitis C using the sickness impact profile. Clin Ther 1994; 16:334343.

8. Silverstein A, Siltzbach LE. Muscle involvement in sarcoidosis: asymptomatic, myositis, and myopathy. Arch Neurol 1969;21:235-241.

9. Jamal MM, Cilursu AM, Hoffman EL. Sarcoidosis presenting as acute myositis: report and review of the literature. J Rheumatol 1988; 15:1868-1871.

10. Stjernberg N, Cajander S, Truedsson H, Uddenfeldt P. Muscle involvement in sarcoidosis. Acta Med Scand 1981; 209:213-216. 
11. Nidiry JJ, Mines S, Hackney R, Nabhani H. Sarcoidosis: a unique presentation of dysphagia, myopathy, and photophobia. Am J Gastroenterol 1991; 86: 16791682 .

12. Fonseca GA, Baca S, Altman RD. Acute myositis and dermatitis as the initial presentation of sarcoidosis. Clin Exp Rheumatol 1993; 11: 553-556.

13. Baydur A, Pandya K, Sharma OP, Kanel GC, Carlson M. Control of ventilation, respiratory muscle strength, and granulomatous involvement of skeletal muscle in patients with sarcoidosis. Chest 1993; 103: 396-402.

14. Badr AI, Sharma OP. Pulmonary function. In: James DG, ed. Sarcoidosis and Other Granulomatous Disorders. New York, Dekker, 1994; 247 - 266.

15. Jacobs HM, Luttik A, Touw-Otten FWMM, Melker de RA. The Sickness Impact Profile; results of an evaluation of the Dutch version. Ned Tijdschr Geneesk 1990; $134 ; 1950-1954$.

16. Quanjer PH, Tammeling GJ, Cotes JE, Pederson OF, Peslin R, Yernault J-C. Lung volumes and forced ventilatory flows. Report working party. Standardization of lung function tests. European Commission for Steel and Coal. Official statement of the European Respiratory Society. Eur Respir J 1993; 6 (suppl 16): 5-40.

17. Black LF, Hyatt RE. Maximal respiratory pressures: normal values and relationship to age and sex. Am Rev Respir Dis 1969; 99: 696-702.

18. Wilson SH, Cooke NT, Edwards RHT, Spiro SG. Predicted normal values for maximal respiratory pressures in Caucasian adults and children. Thorax 1984;39: 535-538.

19. Nickerson BG, Keens TG. Measuring ventilatory muscle endurance in humans as sustainable inspiratory pressure. $J$ Appl Physiol: Respirat Environ Exercise Physiol 1982; 52: 768-772.

20. Larson JL, Kim MJ, Sharp JT, Larson DA. Inspiratory muscle training with a pressure threshold breathing device in patients with chronic obstructive pulmonary disease. Am Rev Respir Dis 1988; 138: 689-696.

21. Schrier AC, Dekker FW, Kaptein AA, Dijkman JH. Quality of life in elderly patients with chronic nonspecific lung disease seen in family practice. Chest 1990; 98: 894-899.

22. Patrick DL, Deyo RA. Generic and disease-specific measures in assessing health status and quality of life. Med Care 1989; 27: S217-S232.

23. Macklem PT. The importance of defining respiratory muscle fatigue. $A m$ Rev Respir Dis 1990; 142: 274.

24. Roussos C. Function and fatigue of respiratory muscles. Chest 1985; 88: 124S$131 \mathrm{~S}$

25. Hamilton AL, Killian KJ, Summers E, Jones NL. Muscle strength, symptom intensity, and exercise capacity in patients with cardiorespiratory disorders. $A m \mathrm{~J}$ Respir Crit Care Med 1995; 152: 2021-2031.

26. Dekhuijzen PNR, Gayan-Ramirez G, Decramer M. Does corticosteroid treatment affect the respiratory muscles? Eur Respir J 1993; 6: 465-466.

27. Tobin MJ. Respiratory muscles in disease. Clin Chest Med 1988; 9: 263-286. 
28. Drent M, Van Velzen-Blad H, Diamant M, Hoogsteden HC, Van den Bosch JMM. Relationship between disease presentation of sarcoidosis and $\mathrm{T}$ lymphocyte profile: a study in bronchoalveolar lavage fluid. Chest $1993 ; 104$ : 795-800.

29. Shijubo N, Imai $\mathrm{K}$, Shigehara $\mathrm{K}$, et al. Soluble intercellular adhesion molecule1 (ICAM-1) in sera and bronchoalveolar lavage fluid of patients with idiopathic pulmonary fibrosis and pulmonary sarcoidosis. Clin Exp Immunol 1994; 95: 156161.

30. Asano M, Minagawa T, Ohmichi M, Hiraga $Y$. Detection of endogenous cytokines in sera or in lymph nodes obtained from patients with sarcoidosis. Clin Exp Immunol 1991; 84: 92-96.

31. Rizzato G, Montemurro L. The locomotor system. In: James DG, ed. Sarcoidosis and Other Granulomatous Disorders. New York, Dekker, 1994; 343-373.

32. Ost D, Yeldandi A, Cugell D. Acute sarcoid myositis with respiratory muscle involvement: case report and review of the literature. Chest 1995; 107:879-882.

33. Pandya KP, Klatt EC, Sharma OP. Sarcoidosis and the diaphragm. Chest 1988; 94:223.

34. Decramer M, Lacquet LM, Fagard R, Rogiers P. Corticosteroids contribute to muscle weakness in chronic airflow obstruction. Am J Respir Crit Care Med 1994; 150: 11-16.

35. Weiner P, Azgad Y, Weiner M. The effect of corticosteroids on inspiratory muscle performance in humans. Chest 1993; 104: 1788-1791.

36. Gallagher CG. Respiratory steroid myopathy. Am J Respir Crit Care Med 1994; 150: 4-5

37. Bradvik I. Use of lung function tests in sarcoidosis. Sarcoidosis 1996; 13:59-62.

38. Karetzky $\mathrm{M}$, McDonough $\mathrm{M}$. Exercise and resting pulmonary function in sarcoidosis. Sarcoidosis 1996; 13: 43-49. 
Chapter 8

Association of fatigue with an acute phase response in sarcoidosis 


\section{Association of fatigue with an acute phase response in sarcoidosis}

M. Drent*, R.M. Wirnsberger*, J. de Vries**, M.P. van Dieijen-Visser***, E.F.M. Wouters*, A.M.W.J. Schols*

*Dept of Pulmonology and ***Dept of Clinical Chemistry, University Hospital Maastricht, **Dept of Psychology, Tilburg University, The Netherlands

Eur Respir J: accepted for publication 


\begin{abstract}
The pathophysiological explanation for one of the most common symptoms in sarcoidosis, i.e. fatigue, still has to be elucidated. It was hypothesized that the presence of fatigue is associated with an acute phase response in sarcoidosis.

A cross-sectional study was performed in 38 sarcoidosis patients. Resting energy expenditure (REE) was measured in the fasting state by indirect calorimetry using a ventilated hood and adjusted for fat-free mass (FFM).

Patients with fatigue $(\mathrm{n}=25)$ suffered also more from other symptoms, e.g. exercise intolerance $(p=0.01)$, the necessity for sleep $(p=0.02)$ and weight loss $(p=0.01)$ compared to those without fatigue $(n=13)$. However, no relationship was found between fatigue and serum angiotensin converting enzyme (sACE) or lung function test results. Patients with fatigue had a higher C-reactive protein (CRP) $\left(11.4 \pm 6.8 \mu \mathrm{g} \cdot \mathrm{mL}^{-1}, \mathrm{p}<0.0001\right)$ and REE adjusted for FFM $\left(33.0 \pm 3.7 \mathrm{kcal}^{\mathrm{kg}} \mathrm{kg}^{-1} \mathrm{FFM}, \mathrm{p}<0.003\right)$ compared to those without fatigue $(3.2 \pm 2.2$ $\mu \mathrm{g} . \mathrm{mL}^{-1} ; 29.2 \pm 2.8 \mathrm{kcal} . \mathrm{kg}^{-1}$ FFM). Furthermore, REE/FFM was significantly related to CRP ( $r=0.54, p=0.001)$.

This study confirms the presence of an acute phase response indicated by metabolic derangements and a moderate increase of CRP in sarcoidosis, particularly in those patients with constitutional symptoms. Future studies should focus on the clinical relevance and therapeutic implications of these findings.
\end{abstract}

\title{
Introduction
}

Sarcoidosis is a multiorgan disorder of unknown origin, characterised by a Tlymphocyte mononuclear phagocyte infiltration in the affected organs, granuloma formation, and distortion of the normal microarchitecture, probably 
antigen driven [1]. The clinical manifestations of sarcoidosis are largely nonspecific, dependent on the intensity of the inflammation and organ systems affected, of which the lung is the most prominent. Patients with sarcoidosis generally present to physicians symptoms directly related to the chest such as coughing, dyspnea, particularly with exertion, and chest pain. Futhermore, they may suffer from nonspecific constitutional complaints such as fever, weight loss, fatigue, anorexia, and malaise [2]. Based on the immunological concept a number of serological parameters gauging the inflammatory activity of sarcoidosis have been determined [3-5]. Despite a thorough history and physical examination, blood and serologic tests, imaging procedures $[2,4]$ and physiologic tests, a specific diagnosis most often requires the use of specialized procedures such as fiberoptic bronchoscopy with bronchoalveolar lavage (BAL) $[2,6]$, transbronchial biopsy, and thoracoscopic or surgical lung biopsy $[2,6]$.

While there is no single test that reflects accurately the progression of sarcoidosis, medical history, clinical examination, chest roentgenograms, and lung function tests are used to evaluate and monitor the disease process [2-5]. When features of disease activity, for example radiologic abnormalities, lung function impairment, and serum angiotensin converting enzyme ( $\mathrm{SACE}$ ) are found to be normalized, rather nonspecific and otherwise difficult to objectify symptoms, such as fatigue and general weakness, may persist [5,7]. Previously, we found that sarcoidosis patients with normal lung function tests, demonstrated reduced respiratory muscle strength and endurance time [8]. Correlations were found between these measures and the most common reported complaint fatigue, in addition to impairment in health status. However, no such relation was demonstrated with sACE levels [8].

Pathognomic criteria or a "golden standard" for monitoring disease activity of sarcoidosis does not exist $[2,4,5]$. Therefore, searching for other parameters useful to monitor disease activity, is still of benefit. Moreover, even though many patients will not require treatment because the symptoms are not 
physically disabling and frequently remit spontaneously, a characterisation of the activity is necessary in order to monitor the course of the disease, and to indicate the necessity to initiate corticosteroid or other treatment [2,3]. Similar to systemic infections, extensive tissue damage due to hypersensitivity or autoimmune reactions is capable of inducing an acute phase response [9]. This acute phase response is characterized by hypermetabolism and the elevation of acute phase proteins such as C-reactive protein (CRP), which are produced in the liver $[9,10]$. Based on the fact that sarcoidosis can be considered as an inflammatory disease together with observations in other inflammatory conditions, we hypothesized the presence of metabolic alterations and a hepatic acute phase response in sarcoidosis patients associated with constitutional symptoms such as fatigue. Furthermore, the impact of fatigue on clinical parameters used to assess disease activity in sarcoidosis was evaluated.

\section{Materials and methods}

\section{Subjects}

Thirty-eight out-patients with newly suspected untreated sarcoidosis were included in this study. The diagnosis of sarcoidosis was based on consistent clinical features, together with bronchoalveolar lavage (BAL) fluid analysis results [6]. Moreover, all patients had a biopsy confirmation of sarcoidosis. The clinical symptoms of the respective patients varied from none (sarcoidosis detected on routine chest radiography) to more or less severe respiratory symptoms or erythema nodosum and arthralgia (i.e. Löfgren's syndrome). None of the subjects participating, had any significant medical history or co-morbidity. Moreover, cultures of the BAL fluid samples obtained were all negative.

To be informed about the presence of constitutional symptoms, the patients participating completed a symptom questionnaire [11] under supervision of a study assistant, to ensure correct answers and to avoid omission of data. Moreover, the 
quality of life (QOL) was evaluated. Therefore, a broad-ranging QOL instrument, the World Health Organization QOL assessment instrument (WHOQOL-100; Dutch version), was employed [12]. The WHOQOL-100 consists of 100 items assessing 24 facets of QOL within six domains and a global evaluative general QOL facet. To measure and qualify fatigue in the present study, only the facet energy and fatigue from the domain physical health was evaluated. Higher scores represent a better QOL for this facet [12]. Written informed consent was obtained from all subjects.

\section{Serologicalmeasurements}

In the course of the initial diagnosis the serological parameters serum angiotensinconverting enzyme ( $\mathrm{SACE}$ ) and serum C-reactive protein (CRP) were assessed. ACE was measured with a colorimetric method using a testkit from Fujirebio inc. (Tokyo, Japan). SACE acts upon the substrate p-hydroxybenzoyl-glycyl-L-histidy-L-leucine) and separates p-hydroxybenzoyl-glycine. Hippuricase reacts and analyzes phydroxybenzoyl-glycine into p-hydroxybenzoic acid and glycine. Quinoneimine dye is produced by oxidation and condensation of the p-hydroxybenzoic acid and 4 aminoantipyrine using sodium metaperiodate $\left(\mathrm{NalO}_{4}\right)$. Finally, the concentration of quinoneimine dye is quantitatively measured at its absorbance maximum at $505 \mathrm{~nm}$ to evaluate the activity of ACE. The reference normal range under this test method is 9 to $25 \mathrm{U} \cdot \mathrm{L}^{-1}$.

CRP was measured using a turbidimetric method performed on the Beckman synchron CX-7 system (Mijdrecht, The Netherlands, kit 465231). In this test CRP combines with a specific antibody to form insoluble antigen-antibody complexes. The detection limit for CRP of the used turbimetry assay was $2 \mu \mathrm{g} \cdot \mathrm{mL}^{-1}$ with a nomal range of 2 to $9 \mu \mathrm{g} \cdot \mathrm{mL}^{-1}$.

\section{Lung function tests and respiratory muscle strength}

Lung function measurements included forced expiratory volume in one second (FEV) and inspiratory vital capacity (IVC) measured with a pneumotachograph. The transfer factor of the lung for carbon monoxide ( $7 \mathrm{~L}, \mathrm{co}$ ) was measured by the single-breath 
method (Masterlab; Jaeger, Würzburg, Germany). Values were expressed as a percentage of those predicted [13].

Inspiratory and expiratory muscle strength were assessed by measuring maximal respiratory mouth pressures using the method of BLACK and HYATr [14]. Maximal inspiratory mouth pressure $\left(P_{\mathrm{l}, \max }\right)$ was measured at residual volume $(\mathrm{RV})$, whilst maximal expiratory mouth pressure ( $\left.P_{\mathbb{E}, \max }\right)$ was measured at total lung capacity (TLC). The equipment used was a pressure transducer (model MP 45-30; Validyne Engineering Corp., Northridge CA, USA). All signals were recorded on a strip chart (type BD 31; Kipp \& Zonen, Delft, The Netherlands). A needle was placed in the proximal end of the mouthpiece to ensure that efforts were performed with open glottis. At least five manoeuvres were performed, until the three highest values were within $5 \%$ of each other. All subjects were seated in upright position and were wearing a noseclip while performing the tests, and the same instructions were given to each subject by one investigator. Values are expressed as absolute terms and as percentages of predicted values according to WILSON et al. [15].

\section{Chestradiographs}

Chest radiographs were made in the posterior-anterior and lateral projections, and were classified by a single experienced reader, blinded to the patient's clinical history, in a standard manner according to the radiographic stage (0 to IV) [16].

\section{Metabolic measures}

Body composition was measured by single frequency bioelectrical impedance analysis (RJL systems, Detroit, USA) in the supine position on the right side. Fat-free

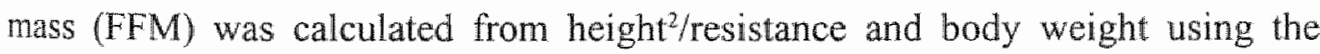
Lukaski formula. In order to assess the degree of functional tissue depletion, FFM was adjusted for body size by calculating the FFM-index (=FFM $(\mathrm{kg})$.height ${ }^{-2}\left(\mathrm{~m}^{2}\right)$ ). Fat mass was calculated by subtracting FFM from body weight.

Resting energy expenditure (REE) was measured after an overnight fast under standardized conditions [17] by indirect calorimetry using a ventilated hood (Oxycon 
beta@, Mijnhardt, Bunnik, The Netherlands). REE was adjusted for FFM and gender, by analysis of covariance [18].

\section{Statistical analysis}

Results are presented as mean \pm SD unless stated otherwise. Pearson"s and, if appropriate, partial (gender was partialed out) correlation coefficients (r) were computed to assess the mutual relationship between the various tests. The Chi-squared test was used for nominal variables. To assess gender differences between the studied groups a Student's $t$-test was used. To examine differences between the sarcoidosis groups with and without fatigue with respect to medical data, analysis of variance was applied with gender as covariate. Analyses of variance (gender as covariate) was also used to compare patients with and without exercise intolerance and patients with and without weight loss with regard to REE adjusted for FFM. In all tests a probability value of less than 0.05 was considered to be statistically significant. All analyses were performed using the Statistical Package for Social Science (SSPS) for Windows.

\section{Results}

The demographic and clinical characteristics, laboratory test results, lung function tests, and body composition of the patients suffering from sarcoidosis studied $(\mathrm{n}=38)$ are summarized in table $I$.

Fatigue was assessed by the corresponding facet of the WHOQOL-100 domain physical health [12]. Patients with fatigue scored significantly lower compared to those patients who did not suffer from fatigue with respect to the facet energy and fatigue $(9.0 \pm 1.5$ and $14.9 \pm 1.8$, respectively; equal variance $t-$ value $10.3, \mathrm{p}<0.0001)$. The patient population was divided into two groups, according to the presence of fatigue. Group I $(n=13)$ consisted of patients who did not suffer from fatigue. Group II $(n=25)$ consisted of sarcoidosis patients who suffered from fatigue. 
Both groups differed with respect to other constitutional symptoms as well (see figure 1). Patients with fatigue suffered more from dyspnea $\left(\chi^{2}(1)=6.3\right.$, $p=0.01)$, exercise intolerance $\left(\chi^{2}(1)=6.9, p=0.009\right)$, muscle pain $\left(\chi^{2}(1)=10.2\right.$, $p=0.001)$, eye problems $\left(\chi^{2}(1)=4.1, p=0.04\right)$ and the necessity for sleep $\left(\chi^{2}\right.$ $(1)=3.9, p=0.05)$ compared to patients without fatigue. These respective groups did not differ with respect to age, smoking history, use of corticosteroids, radiographic stage, sACE levels or lung function test results.

Group II, patients with fatigue, reported more weight loss (number of $\mathrm{kg}$, $F(1)=7.9, p=0.02)$, had a higher serum CRP level compared to group $I(F(1)=15.4$, $\mathrm{p}<0.0001$ ), a higher $\mathrm{REE}(\mathrm{F}(1)=4.3, \mathrm{p}=0.05)$, a higher $\mathrm{REE}$ adjusted for $\mathrm{FFM}$ $(F(1)=9.5, p=0.004)$, as well as fat mass expressed as a percentage of weight $(F(1)=13.4, p=0.001)$ and BMI $(F(1)=4.1, p=0.05$ (table I). No difference was demonstrated in FFM.

$\square$ Group I: no fatigue $\square$ Group II: with fatigue

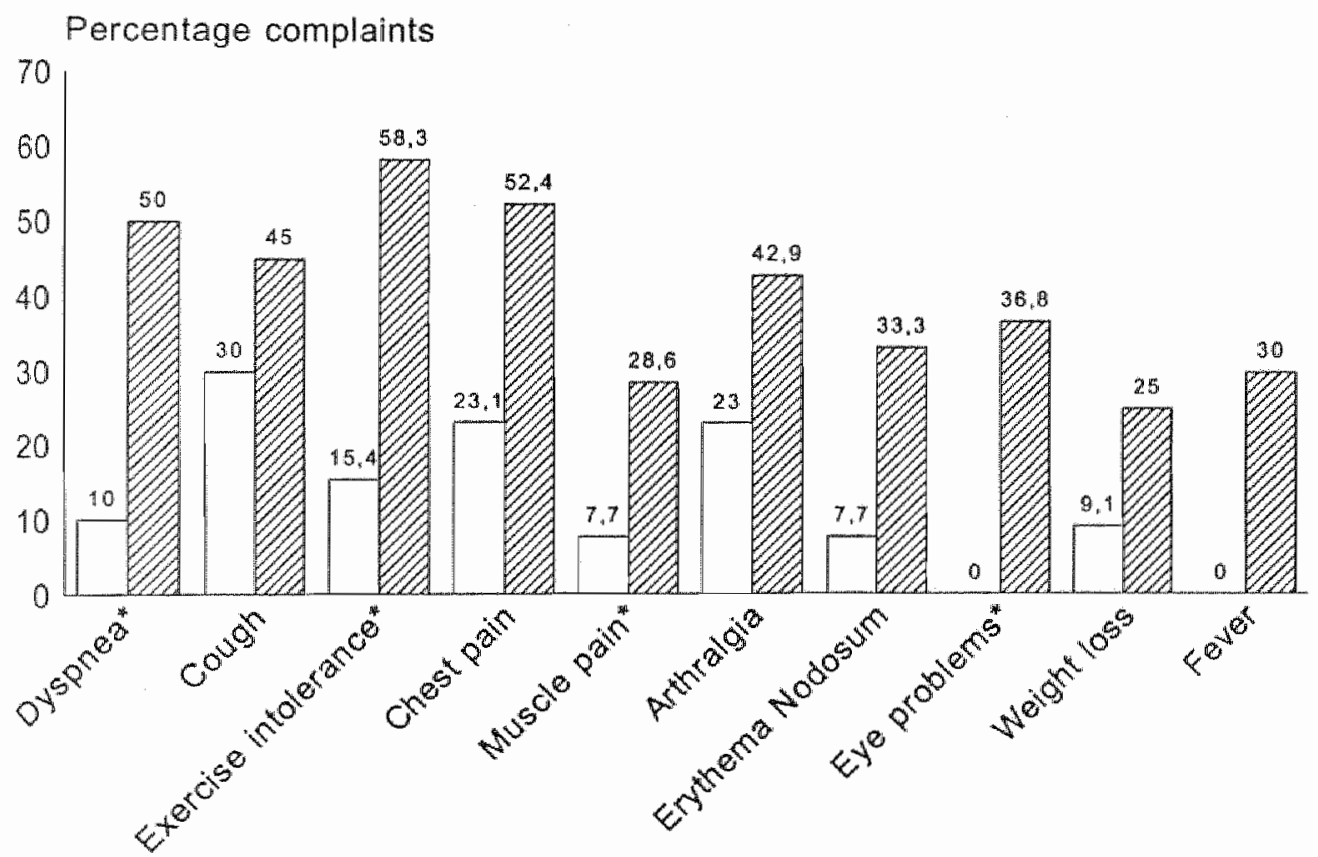

Fig. 1. - Most relevant reported symptoms in sarcoidosis. Group I: patients without fatigue, and group II: patients suffering from fatigue, respectively. * $p<0.05$ group I $v$ s group II. 


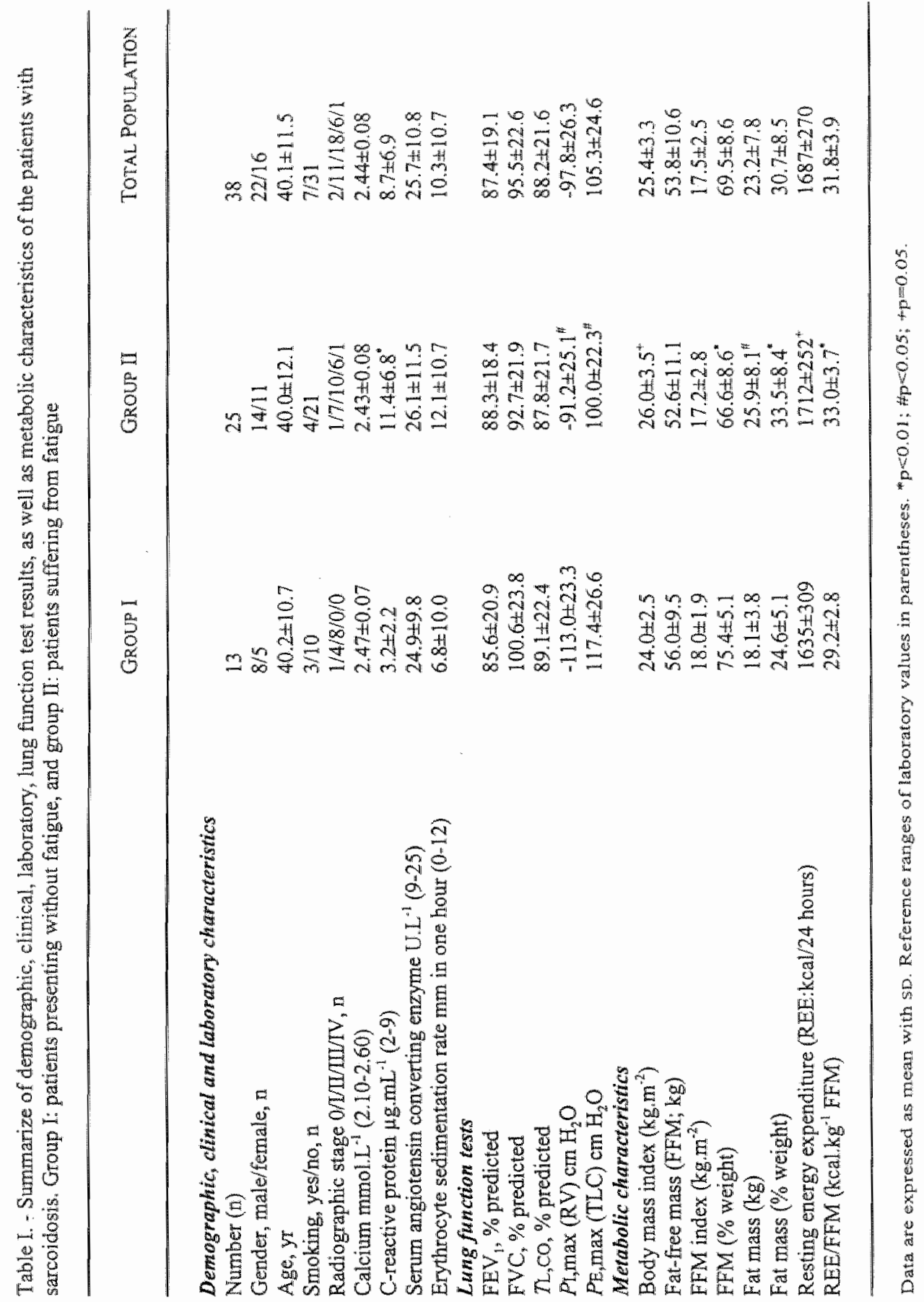


However, FFM calculated as the percentage of the body weight was lower in the group with fatigue $(\mathrm{F}(\mathrm{l})=10.5, \mathrm{p}=0.0003)$.

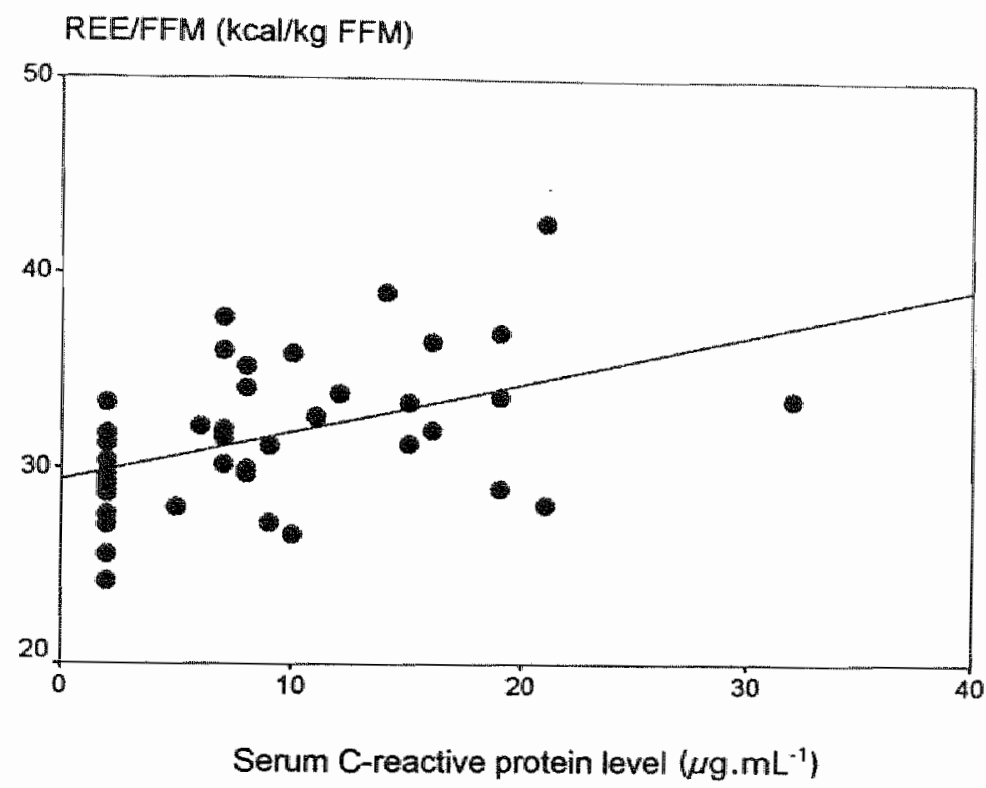

Fig. 2. - Relationship between resting energy expenditure (REE) in kcal adjusted for the fat free mass ( $F F M, k g)$ and C-reactive protein in $\mu \mathrm{g}, \mathrm{mL}^{-1}(\mathrm{r}=0.54, \mathrm{p}=0.001)$.

Furthermore, the $P \mathrm{t}, \max (\mathrm{F}(1)=5.4, \mathrm{p}=0.04)$ appeared to be more impaired in group II. Moreover, REE divided by FFM was significantly associated with $\mathrm{CRP}$ ( $\mathrm{r}=0.54, \mathrm{p}=0.001$; figure 2 ), and significantly higher in patients suffering from weight loss $(F(1)=5.2, p=0.03)$ or exercise intolerance $(F(1)=12.3$, $\mathrm{p}=0.001$ ). Exercise intolerance also appeared to be related to increased body temperature $\left(\chi^{2}(1)=5.7, p=0.02\right)$. The radiographic stage was positively related to REE divided by FFM $(r=0.39, p=0.02)$. However, no relationship was found between the radiographic stage and lung function test results nor with $\mathrm{SACE}$ or any other laboratory finding listed in table $I$. 


\section{Discussion}

This study is the first to describe a relationship between one of the most common symptoms in sarcoidosis, i.e, fatigue and the presence of acute phase response evidenced by hypermetabolism and an increase of the serum Creactive protein (CRP) level to a limited extent. Moreover, patients suffering from fatigue were characterized by a different body composition compared to those patients not suffering from fatigue. No relationship was found between fatigue and commonly used parameters to monitor disease activity in sarcoidosis such as the SACE level, radiographic findings, and lung function tests. Only a negative relationship between the radiographic stage and the fatfree mass was demonstrated. Clearly, there is a wide spectrum of tissue inflammation and organ dysfunction within, as well as between, each radiographic stage. KARETZKY and MCDONOUGH found that the magnitude of functional impairment may vary widely from the apparent histopathological involvement as reflected by chest radiography and lung volumes [19]. There is little true incidence data in the literature regarding metabolic alterations in patients with sarcoidosis. Only one report - including twelve patients having various interstitial lung diseases (ILD), of which only three were suffering from sarcoidosis - demonstrated the presence of an elevated REE [20].

Increased CRP production is a nonspecific response to most forms of tissue inflammation [21,22]. Hepatocytes have been identified as the site of origin of acute phase proteins such as CRP. Thus, the liver plays an important role in the acute phase response $[10,23]$. The CRP response - although rather minor - was present in the majority of patients and appeared indicative of the inflammatory activity of sarcoidosis. Similar responses - resulting in a slightly elevated or normal CRP - are reported in inflammatory disorders such as systemic lupus erythematosus, morbus Crohn, and ulcerative colitis [24]. In contrast, an intercurrent microbial infection provokes a major CRP response [10]. To date, 
HND et al. found in their study population an increased CRP in two out of nine patients with active sarcoidosis $(22.2 \%)$, but in none out of the five inactive sarcoidosis patients [25].

Most clinical manifestations of sarcoidosis are secondary to the direct effect of the accumulation of activated immunocompetent cells in the tissues involved - notably helper $\mathrm{T}$ lymphocytes and macrophages $[2,5,9,26,27]$. The activation of cells of the monocyte/macrophage lineage is compartmentalised. Alveolar macrophages have been identified to release tumor necrosis factor- $\alpha$ (TNF- $\alpha$ ), interleukine-1 (IL-1), and prostaglandin $\mathrm{E}_{2}$ spontaneously in the course of sarcoidosis, whereas the corresponding cells of the peripheral blood are quiescent $[5,26]$. In the present study we did not measure cytokine levels, but as would be expected, we found a significant relationship between the elevated CRP levels and resting metabolic rate. The clinical significance of this relationship was indicated by the fact that REE and CRP were significantly higher in patients suffering from fatigue. In line with this, we previously found that the serum CRP level was related to fatigue in patients with an autoimmune disease, i.e. rheumatoid arthritis (RA). Patients suffering from RA with a high serum CRP level reported to be significantly more impaired by fatigue assessed by the WHOQOL-100 facet energy and fatigue [12] - compared to those with a normal serum CRP level (unpublished data). In a part of the hypermetabolic patients with COPD [17], increased resting metabolic rate seems to be related to the level of systemic inflammation. Furthermore, patients with fatigue were characterized by a significantly higher fat mass, whereas fat free mass as percentage of body weight was significantly decreased in this group. These body compositional changes suggest a preferential loss of FFM in the latter group. The findings of the present study could indicate a catabolic response in a subgroup of sarcoidosis patients which may be consistent with the hypothesis that, during an acute phase response, cytokines redirect host protein metabolism away from peripheral tissue such as muscle, and towards the liver. The higher fat mass in the subgroup of sarcoidosis patients suffering from 
fatigue, however, could - in view of other experienced symptoms - also be associated with a disease related impairment of the functional capacity [26, 31]. Moreover, under those circumstances one might also expect a higher body weight and perhaps weight gain in the patients with symptoms, whereas on the contrary more often weight loss was reported. Nevertheless, a decreased fatfree mass in turn may affect respiratory muscle strength which in the present study as well as in a previous study was indeed found to be significantly reduced [8]. Furthermore, we previously reported that patients with sarcoidosis are limited in physical functioning [8].

It has generally been assumed that the marked loss of body proteins resulting in a loss of body mass - initiated by TNF- $\alpha$ and leukocyte pyrogen or IL-1, mediated by prostaglandin $\mathrm{E}_{2}$ - occurs primarily in the skeletal muscle, which constitutes the major protein reserve of the organism [23]. Net protein breakdown in muscle can also provide amino acids for synthesis of acute phase proteins by the liver and for the synthesis of immunoglobulins, commonly increased in sarcoidosis $[2,4,23]$. Leukocyte pyrogen also causes induction of the acute phase proteins and the enhancement of lymphocyte proliferation [23, 26]. Moreover, it is well established that prostaglandin $E_{2}$ is a very potent stimulator of peripheral pain receptors and hyperalgia [23]. In immune disorders such as sarcoidosis and RA, immune derived cytokines initiate an acute phase response, which is also characterized by fever, inactivity, muscle pain, fatigue, anorexia, and catabolism [23, 32]. An appreciation of the processes of the acute phase reaction is likely to be of clinical relevance. For example, the recent demonstration that muscle breakdown, like fever, is brought about by IL-1 via an intermediate prostagland in step, suggests that anti-prostaglandin agents may not only cause defervescence but might help to prevent muscle breakdown [10]. Therefore, administration of non-steroidal anti-inflammatory drugs - as alternative to steroids - might be of benefit in the treatment of sarcoidosis as it has been suggested that these drugs lower rates of muscle proteolysis [3, 5, 23]. Future studies are needed to evaluate this suggestion. 
In conclusion, this study indicates that an acute phase response-evidenced by metabolic derangements and a moderate increase of the serum C-reactive protein level - is involved in sarcoidosis. The resting energy expenditure and serum C-reactive protein level appeared to quantify and characterize the constitutional impairment and inflammation in patients with sarcoidosis. In addition, constitutional symptoms, e.g. fatigue, that are otherwise difficult to objectify accurately, were found to be related to the resting energy expenditure as well as the serum C-reactive protein level. Therefore, assessment of the serum C-reactive protein level and resting energy expenditure adjusted for body composition may be useful in the management of sarcoidosis. Further investigations - including cytokine determination - are needed to thrash out the clinical importance of the acute phase response associated with inflammation and related symptoms, such as fatigue, in sarcoidosis.

\section{Acknowledgements}

The authors would like to thank Helma Voets, Alwin de Vries and Gerard Pronk for their great help in collecting the data, as well as Marjon Elfferich for her advice during the preparation of this manuscript.

\section{References}

1. Thomas PD, Hunninghake GW. Current concepts of the pathogenesis of sarcoidosis. Am Rev Respir Dis 1987; 135; 747-760.

2. Sharma OP, Alam S. Diagnosis, pathogenesis and treatment of sarcoidosis. Curr Opin Pulm Med 1995; 1: 392-400.

3. Hunninghake $\mathrm{GW}$, Gilbert $\mathrm{S}$, Pueringer $\mathrm{R}$, et al. Outcome of the treatment of sarcoidosis. Am J Respir Crit Care Med 1994; 149: 893-898.

4. Baudouin SV, du Bois RM. Disease activity. In: James DG, ed. Sarcoidosis and Other Granulomatous Disorders. New York, Dekker, 1994; 153-180.

5. Müller-Quernheim J. The cytokine network in sarcoidosis. Eur Cytokine Netw $1996 ; 7: 13-26$. 
6. Drent M, Van Nierop MAMF, Gerritsen FA, Wouters EFM, Mulder PGH. Computer program using BALF analysis results as diagnostic tool in interstitial lung diseases. Am J Respir Crit Care Med 1996; 153: 736-741.

7. James DG. Complications of sarcoidosis. Chronic fatigue syndrome. Sarcoidosis 1993; 10:1-3.

8. Wirnsberger RM, Drent $M$, Hekelaar $N$, et al. Relationship between respiratory muscle function and quality of life in sarcoidosis. Eur Respir $J 1997 ; 10: 1450$ 1455 .

9. Anisman H, Baines MG, Berczi I, et al. Neuroimmune mechanisms in health and disease: 2. Disease. Can Med Assoc J 1996; 155(8): 1075-1082.

10. Highton J. The acute phase response: a clinical perspective. Aust NZJMed 1984; 14:173-178.

11. Wirnsberger RM, De Vries J,Wouters EFM, Drent M. Clinical Presentation of Sarcoidosis in the Netherlands. An Epidemiological Study. Neth JMed 1998; 53 : $53-60$.

12. Wirnsberger RM, De Vries J, Breteler MHM, Van Heck GL, Wouters EFM, Drent M. Evaluation of quality of life in sarcoidosis patients. Respir Med 1998;92:750756.

13. Quanjer PH, Tammeling GJ, Cotes JE, Pederson OF, Peslin R, Yernault J-C. Lung volumes and forced ventilatory flows. Report working party. Standardization of lung function tests. European Commission for Steel and Coal. Official statement of the European Respiratory Society. Eur Respir J 1993; 6(suppl.16): 5-40.

14. Black LF, Hyatt RE. Maximal respiratory pressures: normal values and relationship to age and sex. Am Rev Respir Dis 1969; 99: 696-702.

15. Wilson SH, Cooke NT, Edwards RHT, Spiro SG. Predicted normal values for maximal respiratory pressures in Caucasian adults and children. Thorax 1984;39: 535-538.

16. DeRemee RA. The roentgenographic staging of sarcoidosis. Historic and contemporary perspectives. Chest 1983; 83: 128-133.

17. Schols AMWJ, Buurman WA, Staal-van den Brekel AJ, Dentener MA, Wouters EFM. Evidence for a relation between metabolic derangements and increased levels of inflammatory mediators in a subgroup of patients with obstructive pulmonary disease. Thorax 1996: 51;819-824.

18. Ravussin $E_{,}$Bogardus $C$. Relationship of genetics, age and physical fitness to daily energy expenditure and fuel utilization. Am J Clin Nutr 1989; 49: 968-975.

19. Karetzky M, McDonough M. Exercise and resting pulmonary function in sarcoidosis. Sarcoidosis 1996; 13:43-49.

20. Fitting $W$, Frascarolo $P$, Jéquier $E$, Leuenberger $P$. Resting energy expenditure in interstitial lung disease. Am Rev Respir Dis 1990; 142: 631-635.

21. Hrycaj $P$, Wurm $K$, Mennet $P$, Müller W. Microheterogeneity of acute-phase glycoproteins in patients with pulmonary sarcoidosis. Eur Respir J 1996; 9: 313 318.

22. Asano M, Minagawa $\mathrm{T}$, Ohmichi M, Hiraga $Y$. Detection of endogenous cytokines in sera or in lymph nodes obtained from patients with sarcoidosis. Clin Exp Immunol 1991; 84: 92-96. 
23. Baracos V, Rodemann HP, Dinarello CA, Goldberg AL. Stimulation of muscle protein degradation and prostaglandin $E_{2}$ release by leukocytic pyrogen (interleukin-1). A mechanism for the increased degradation of muscle proteins during fever. New Engl J Med 1983; 308: 553-558.

24. Fagan EA, Dyck RF, Maton PN, et al. Serum levels of C-reactive protein in Crohn's disease and ulcerative colitis. Eur J Clin Invest 1982; 12:351-359.

25. Hind CR, Flint KC, Hudspith BN, Felmingham D, Brostoff J, Johnson NM. Serum C-reactive protein concentrations in patients with pulmonary sarcoidosis. Thorax 1987; 42: 332-335.

26. De Rose V, Trentin L, Crivellari MT, et al. Release of prostaglandin $\mathrm{E}_{2}$ and leukotriene $\mathrm{B}_{4}$ by alveolar macrophages from patients with sarcoidosis. Thorax $1997 ; 52: 76-83$.

27. Drent M, Van Velzen-Blad H, Diamant M, Hoogsteden HC, Van den Bosch JMM. Relationship between disease presentation of sarcoidosis and T lymphocyte profile: a study in bronchoalveolar lavage fluid. Chest 1993; 104 : 795-800.

28. Buck M, Chojkier M. Muscle wasting and dediffentiation induced by oxidative stress in a murine model of cachexia is prevented by inhibitors of nitric oxide synthesis and antioxidants. EMBO J 1996; 15: 1753-1765.

29. Landay AL, Jessop C, Lennette ET, Levy JA. Chronic fatigue syndrome: clinical condition associated with immune activation. Lancet 1991; 338: 707-712.

30. Voijdani A, Ghoneum M, Choppa PC, Magtoto L, Lapp CW. Elevated apoptotic cell. population in patients with chronic fatigue syndrome: the pivotal role of protein kinase RNA. J Intern Med 1997; 242: 465-478.

31. Evans WJ, Campbell WW. Sarcopenia and age-related changes in body composition and functional capacity. JNutr 1993; 123: 465-468. 
Chapter 9

General discussion 


\section{GENERAL DISCUSSION}

\section{Introduction}

Sarcoidosis patients exhibit a broad variety of clinical features [1]. Patients with pulmonary sarcoidosis may present with symptoms related directly to the chest such as coughing, dyspnea on exertion, retro-sternal chest pain, chest discomfort, and wheezing [2-4]. However, patients may also present to physicians having constitutional symptoms such as fatigue, fever, anorexia, weight loss and/or malaise [3]. Moreover, constitutional symptoms such as fatigue and general weakness appear to be a frequently persistent problem of sarcoidosis patients. These symptoms have had little attention in the sarcoidosis literature [5]. Therefore, in this thesis constitutional symptoms such as fatigue were assessed together with health status and quality of life (QOL) in sarcoidosis patients. In addition, the relationship of symptoms, health status, and QOL with physiological measures was studied.

\section{Summary of the study}

Health status assessed by the Sickness Impact Profile (SIP) [6] as well as QOL assessed by the World Health Organization Quality of Life assessment instrument (WHOQOL-100) [7] appeared to be impaired in patients suffering from sarcoidosis compared to healthy controls [chapter 4,5]. Fatigue was the most frequently reported symptom of sarcoidosis patients [chapter 3,4]. Moreover, symptomatic as well as asymptomatic sarcoidosis patients showed an impairment of QOL with respect to their general health, energy levels, and sleep [chapter 5]. The symptom fatigue and lower levels of energy were not associated with the psychological health of patients [chapter 5]. In addition, patients reported a broad range of persistent physical symptoms [chapter 3]. When comparing QOL in sarcoidosis and rheumatoid arthritis, both patient groups suffered from 
impaired general health, sleeping problems, fatigue, and problems performing their daily activities and their work compared with healthy controls. Rheumatoid arthritis patients showed more impairment of their mobility and were more dependent on medication than sarcoidosis patients [chapter 6]. Although at presentation $37 \%$ of the sarcoidosis patients suffered from chest and muscle pain, and 52\% from arthralgia at presentation [chapter 3], pain was not the most important problem [chapter 6]. Sarcoidosis patients demonstrated reduced strength and endurance time of respiratory muscles compared to healthy controls. Moreover, these latter measures were found to be related to various aspects of health status [chapter 7]. In sarcoidosis patients suffering from fatigue an acute phase response indicated by metabolic derangements and an increase of the serum C-reactive protein (CRP) levels were found. Moreover, these patients reported more other constitutional symptoms and other physical symptoms than patients without fatigue [chapter 8]. No relationships between health status and QOL and routinely performed measures such as serum angiotensin converting enzyme (SACE) and pulmonary function tests were found [chapter $4,5,7]$. The symptom fatigue appeared to be related to increased levels of CRP, weight loss, and a higher resting energy expenditure (REE) adjusted for fat-free mass (FFM) [chapter 8].

\section{Fatigue and other constitutional symptoms in sarcoidosis}

Fatigue is a rather nonspecific symptom. Although fatigue was the most frequently reported symptom, other constitutional symptoms such as reduced exercise capacity, weight loss, fever, sleeping problems, general weakness, arthralgia, muscle pain, and starting problems were also frequently reported by sarcoidosis patients [chapter 3]. In a high percentage of patients these symptoms persisted. It was suggested that in sarcoidosis, as in other immune mediated disorders, constitutional symptoms may be caused by the circulating cytokines such as tumour necrosis factor (TNF)- $\alpha$ and interleukin (IL)-1 [8]. In a number of other conditions in which increased and chronic production of 
cytokines play a role, fatigue also is an important feature. In the chronic fatigue syndrome, fatigue is the cardinal symptom. It has been suggested that cytokines, as present in the cerebrospinal fluid, may mediate the symptoms of chronic fatigue syndrome [9]. TNF- $\alpha$ and IL-1 are, in addition, known to provoke slowwave sleep [10]. In inflammatory disorders of the central nervous system such as multiple sclerosis, fatigue and sleepiness were suggested to be caused by IL1 released by activated macrophages in the brain [10]. In rheumatoid arthritis, also a chronic inflammatory disorder, an improvement of inflammatory activity parameters such as CRP, joint swelling and pain, as well as an improvement of fatigue was found after treatment with anti-TNF- $\alpha$ [11].

As mentioned above, fatigue appeared to be a major problem in patients suffering from sarcoidosis. Quantitative assessment of fatigue and differentiation from depressive symptoms is an important aspect to include in the therapeutic approach. In the last several years questionnaires for the quantitative assessment of fatigue have been developed and applied to medical conditions such as chronic fatigue syndrome [12], multiple sclerosis, and systemic lupus erythematodes [13]. For the assessment of fatigue, the facet energy and fatigue of the WHOQOL-100 appeared to be an appropriate measure in sarcoidosis [chapter 5].

Patients with sarcoidosis may present with rather nonspecific constitutional symptoms. Therefore, it is sometimes difficult to diagnose this disorder. In about $25 \%$ of patients it took more than one year before the diagnosis of sarcoidosis was made. Moreover, in more than $40 \%$ of patients another diagnosis was made before, finally, sarcoidosis was diagnosed correctly [chapter 3]. Therefore, it seems important that besides pulmonologists, physicians in general are aware of the possible presentations of sarcoidosis.

\section{Health status and $Q O L$}

In the present study, a generic health status measure, the SIP [6], and a generic QOL measure, the WHOQOL-100 [7] were employed. Considering the various 
organs which may be involved in sarcoidosis and the broad range of physical symptoms reported by patients, the use of a disease-specific instrument for respiratory diseases was not considered appropriate.

Especially in the medical literature, the terms health status, health-related QOL, and QOL are used in a confusing way, and are often not clearly distinguished. Health status measures assess mainly functioning, through the use of frequency (How often...?) and capacity (Are you able to...?) questions. In contrast, QOL instruments assess the individual's subjective perception of his/ her life in a broad way using also evaluation questions (How satisfied are you...?). However, measures that assess health status often claim to evaluate QOL [14]. Also in the present study, in the two articles that were written first, we have used the term QOL, although a health status measure, the SIP, was employed. Thereafter, in the further course of the study, when actually QOL was assessed in sarcoidosis patients, using the WHOQOL-100, the term QOL was properly defined and the correct use of it was applied. However, there remains a certain mixing of terms, e.g., when quoting authors who report "QOL" data, when they actually have used a health status measure.

To the best of our knowledge, health status and QOL have not been assessed in sarcoidosis patients, previously. In the present study, health status [chapter 4,7 ] as well as QOL [chapter 5,6] were found impaired in sarcoidosis patients. In another chronic inflammatory disorder, rheumatoid arthritis, subjective stressors such as pain, perceived limitation, and dependence were found only weakly related to traditionally used disease status measures [15]. All three stressors were related to patients' well-being [16]. As for rheumatoid arthritis [17], for chronic respiratory diseases such as chronic obstructive pulmonary disease (COPD), disease-specific health status measures have been developed $[18,19]$, but also generic health status measures such as the SIP are employed for the assessment of impact of the disease on patients' lives. In COPD, the relationship between health status, assessed by these questionnaires and physiological measures of disease severity such as pulmonary function tests has 
been found to be weak $[18,20-23]$. However, self-reported respiratory symptoms such as dyspnea, wheezing, and subjective severity of breathing problems were found related to health status $[22,24]$. The symptom dyspnea was related not only to the physical but also to the psychosocial functioning of patients, measured by the $\operatorname{SIP}[6,23,24]$.

In the present study, as might be expected, health status as well as QOL of sarcoidosis patients were found more severely impaired in patients with current symptoms than in those without current symptoms [chapter 4,5]. The symptomatic patients suffered from impaired QOL with respect to their mobility, working capacity, and activities of daily living. Moreover, they showed low levels of positive feelings and problems with recreation compared with the asymptomatic group. However, no differences were found between patients with and without current symptoms with respect to their impairment of general health and overall QOL, sleeping problems, and fatigue. Thus, also sarcoidosis patients who reported no symptoms were impaired with respect to their QOL and health status, compared to healthy controls [chapter 5]. These findings indicate, that evaluation of symptoms without attention for QOL and health status may lead to misinterpretation of the patients' well-being. It should be noted that by using the WHOQOL-100 (a QOL instrument) a number of aspects of life that are not assessed by a health status measure were incorporated. Other aspects of QOL such as, for instance, environmental and spiritual aspects can be evaluated using a broad QOL instrument. Of special importance for this study was the assessment of the symptom fatigue by the WHOQOL-100. For the evaluation of the clinical status, the assessment of both health status and QOL should be included in the management of sarcoidosis patients.

To date, De VRIEs et al. [25] demonstrated that the group of out-patients from the eight Dutch hospitals showed only minimal differences of QOL compared to the patients studied in the survey of members of the Dutch Sarcoidosis Society, when corrected for age, gender, and current symptoms. 
Psychological factors and depressive symptoms

As mentioned by KLONOFF and KLEINHENZ, patients with sarcoidosis may present with depressive symptoms and anxiety, together with vague physical complaints [26]. The sarcoidosis patients in this latter study, as a group, were not found to suffer from clinical significant depression. In line with this, we did not find the criteria for clinical depression in the sarcoidosis patient population of the present study. After dividing the study population into patients with and without current symptoms, symptomatic patients appeared to have more depressive symptoms [chapter 4]. In addition, patients who were less satisfied with their psychological situation were more depressed [chapter 5]. No relationship was found between fatigue and the psychological health of the patients. Depressive symptoms in these patients may be, at least partly, an expression of exhaustion by the ongoing disease. DEREMEE [27] recently discussed the possibility of neuro-humoral agents in sarcoidosis which may explain psychological aspects in sarcoidosis. However, this hypothesis needs future study.

\section{Relationship between the immune system and the brain}

Evidence of complex interactions between the immune system and the brain has been provided in the past several years [28]. The immunosuppressive effect of stressful life events has been reported $[29,30]$. However, for the changes in immunity, a person's coping strategy and personality were found as important as the nature of the life event itself [30]. Psychological stress can compromise the immune system, and, on the other hand, humoral components of the immune system can directly influence brain function and behaviour [31]. For example, in patients suffering from depression, IL-1, interferon (INF)- $\gamma$, and TNF were found increased, while IL-2 was reduced [31,32]. IL-1 was found the most potent inducer of corticotropin releasing factor (CRF) in the hypothalamus $[30,33]$. It has been suggested that the physiological role of increased production of glucocorticosteroids in response to stress is to prevent 
defence mechanisms from overshooting [34]. Although the interactions between immune system and brain are complex and not fully understood, these aspects should have attention in an immune mediated disease like sarcoidosis. Whether patient education aimed at reducing stress and enhancing adequate coping strategies may be effective in the care of sarcoidosis patients needs further investigation.

\section{Physiologicalmeasures}

As SHARma has pointed out, in contrast to other disorders, activity in sarcoidosis does not necessarily indicate a progressive course or a fatal prognosis [4]. In addition, no single test exists for the accurate assessment of disease activity and progression of sarcoidosis. Therefore, the course of the disease is monitored mainly by clinical features, pulmonary function tests, and chest radiographs [4]. However, these tests do not always reflect the clinical situation and well-being of patients, as demonstrated in the present study.

The broad impact of sarcoidosis on patients' QOL in the present study is remarkable considering the relatively slight impairment in pulmonary function tests of the patients. Pulmonary function tests of the out-patients from the eight participating Dutch Hospitals as well as those of the newly diagnosed patients recruited from the Academic Hospital Maastricht, except one, were either normal or showed only little impairment. Besides the conventional tests for disease activity, the assessment of other functional parameters may be indicated in diagnostic approach and follow-up of sarcoidosis patients. In the present study, strength and endurance time of respiratory muscles were low in a subgroup of this study population compared with healthy controls. However, no weakness of peripheral muscles was found in these sarcoidosis patients. Whether weakness and fatigue of respiratory muscles are an expression of the involvement of respiratory muscles by sarcoidosis remains unclear. Others have found lower $P_{E}, \max$, and $P_{1, \max }$ and respiratory muscle dysfunction in sarcoidosis as well $[35,36]$. 
In patients with COPD, muscle weakness and fatigue of respiratory and peripheral muscles is well known. However, in patients with COPD a number of factors contribute to this feature, such as hypoxaemia, hypercapnia, muscle depletion, decreased blood supply to the muscles, drugs, and electrolyte abnormalities $[37,38]$. In our patient population, it is unlikely that these factors are involved. Moreover, in COPD a close relationship was found between the intensity of dyspnea and respiratory muscle effort [39]. In COPD patients, respiratory and peripheral muscle weakness, but not pulmonary function, was found associated with high medical consumption [40]. A high percentage of the patient members of the Dutch Sarcoidosis Society (70\%) and of the symptomatic patients recruited from the hospitals (54\%) reported dyspnea. Of the latter group the pulmonary function data were available. As mentioned above, pulmonary function tests such as $\mathrm{FEV}$, and $T \mathrm{~L}, \mathrm{Co}$ were normal or only mildly impaired in these patients. However, more than half of these patients suffered from dyspnea. Therefore, the symptom dyspnea in sarcoidosis patients needs to be evaluated in more detail. However, dyspnea is a highly subjective symptom and, as such, difficult to evaluate. Physiological factors other than pulmonary function impairment, or psychological factors may contribute to patients' perception of dyspnea. Presumably, factors related to the perception of dyspnea of sarcoidosis patients may include chest pain and fatigue, as chest pain as well fatigue and low levels of energy, assessed by the WHOQOL-100, appeared to be related to the symptom dyspnea (unpublished data).

As mentioned above, a relatively high percentage of patients suffered from persistent muscle pain. Although asymptomatic involvement of muscles in sarcoildosis has been reported in up to $80 \%$ of cases [41], symptomatic involvement is thought to be much less frequent $[41,42]$. Symptomatic muscle involvement that has been described includes palpable nodules, acute myositis, and chronic myopathy [41-43]. Patients with granulomatous muscle involvement usually present with pain, weakness, and muscle atrophy [41]. The latter two features were not reported in our study population. In the present study, 
peripheral muscle strength was not decreased compared to healthy controls in a subgroup of the patients recruited from the hospitals [chapter 7]. The isolated muscle pain may be an expression of otherwise asymptomatic involvement of the muscles by sarcoidosis. However, it seems possible, that the muscle pain, together with other constitutional symptoms, is an expression of the systemic inflammation. In the present study, patients who suffered from current fatigue, also reported more other constitutional symptoms, including muscle pain [chapter 8]. As discussed above, in rheumatoid arthritis, another inflammatory disease, constitutional symptoms, together with pain, improved after treatment with anti-TNF- $\alpha$. Whether in sarcoidosis, these symptoms may also be related to high serum and tissue levels of cytokines remains speculative and needs further study.

In the chronic fatigue syndrome, in which the immune system is also thought to be compromised [44], patients reported reduced exercise capacity and muscle weakness as well as muscle pain. However, muscle strength and endurance were found to be normal [45]. It has been suggested that these complaints are caused partly by inactivity due to the excessive fatigue. In line with this, in sarcoidosis, symptoms as general weakness and muscle pain may be caused, at least partly, by inactivation due to fatigue and lack of energy. As mentioned above, we did not find weakness of peripheral muscles in a subgroup of our study population [chapter 7]. However, these parameters were assessed in only a small number of patients. Whether exercise training and muscle strengthening programs may be effective in sarcoidosis patients needs further evaluation. In inflammatory neuromuscular disorders, where weakening of muscles is caused by severe restriction of activity, muscle strengthening programs have shown positive effects $[46,47]$. As exacerbation of inflammation after muscle strength training has been reported, the importance of a careful design and supervision of strength training programs in patients with inflammatory muscle disorders has been stressed [46-48]. Considering this treatment modality for sarcoidosis patients, programs should meet the same criteria. 
An acute phase response, evidenced by a moderate increase ofCRP level and hypermetabolism was found in sarcoidosis patients who suffered from current constitutional symptoms such as fatigue [chapter 8]. In other chronic inflammatory disorders such as ulcerative colitis CRP levels were found moderately ellevated as well, while these levels were higher in Crohn's disease and rheumatoid arthritis [49-51]. In rheumatoid arthritis patients we found a relationship between elevated CRP levels and fatigue [chapter 6]. In inflammatory bowel disease, superimposed infection may account for higher levels of CRP [49]. In sarcoidosis, current infections do not seem to be involved, and even slightly elevated CRP levels appeared to reflect disease activity of patients with persistent constitutional symptoms. In patients who suffered from fatigue and weight loss, a higher resting energy expenditure (REE) adjusted for fat-free mass (FFM) was found. Moreover, patients with fatigue showed lower percentages of FFM. Reduction of FFM is an expression of muscle wasting. The muscle wasting of cachexia in chronic diseases is thought to be mediated by TNF- $\alpha$, possibly together with other cytokines [52-54]. Also in COPD, in patients with an increase of REE, elevated serum levels of cytokines such as TNF and elevated levels of CRP were found [55]. The weight loss, depletion of FFM, and increased REE, found in patients with COPD [56], was attributed at least partly to inflammation in these patients [55]. Previously, Pouw et al. found disturbances in muscle energy metabolism under resting conditions in patients with stable severe COPD [57].

In sarcoidosis, the energy balance should be monitored together with the inflammatory status of patients. More important, further research is needed with respect to metabolic changes in this disorder.

Relationships between physiological measures and health status and $Q O L$ For the follow-up of patients suffering from sarcoidosis, chest radiographs and pulmonary function tests are routinely performed [58]. Moreover, in the clinical practice, although relatively unspecific, $\mathrm{SACE}$ is assessed as well. In the present 
study, no relationships of SACE and pulmonary function tests with health status and QOL were found [chapter 4,5]. The radiographic stage correlated with the health status categories 'alertness behaviour', 'emotional behaviour', 'home management', and 'social interaction' of the SIP. Moreover, respiratory muscle strength and endurance time were found decreased in sarcoidosis patients. Respiratory muscle strength correlated with the presence of symptoms such as fatigue, whereas respiratory muscle endurance time correlated with the subscales 'body care and movement' and 'mobility' of the SIP [chapter 7]. However, no relationship between pulmonary function tests and SACE and aspects of QOL assessed by the WHOQOL-100 were found [chapter 5].

In search for objective measures for the symptom fatigue in patients with sarcoidosis, we found metabolic aberrations in those patients who reported current fatigue. No relationship between the metabolic aberrations and pulmonary function tests was found.

\section{Conclusions}

This thesis showed that health status and QOL of patients with sarcoidosis were significantly impaired compared to healthy controls. As might be expected, patients with current symptoms were more impaired in their QOL than asymptomatic patients. However, also patients who did not report any symptoms spontaneously, appeared to be impaired with respect to their general health, levels of energy and their sleep, assessed by the WHOQOL-100. The facet energy and fatigue proved to be a sensitive measure for the identification and quantification of fatigue, the most frequent symptom in sarcoidosis. Moreover, the use of a broad QOL instrument in sarcoidosis patients adds important aspects which are not assessed by a health status measure. In addition, sarcoidosis patients suffered from a broad range of frequently persistent physical symptoms. Patients with depressive symptoms suffered from more physical symptoms. A considerable number of patients reported disease related strain and coping problems. 
No relationship of health status and QOL measures with most of the routinely performed physiological tests was found. However, lower expiratory muscle strength was related to the symptom fatigue. Moreover, fatigue was related to metabolic alterations of body composition and basal metabolism. Patients who suffered from fatigue suffered from more other constitutional symptoms such as weight loss, fever, exercise intolerance, and other physical symptoms than those who did not report fatigue.

\section{Directions for future research}

The present study has shown the importance of constitutional symptoms, especially persistent fatigue, in patients suffering from sarcoidosis. Particularly for the individual sarcoidosis patient, it is crucial that these symptoms are recognized and taken seriously. The assessment of health status and QOL should be included in the follow-up of sarcoidosis patients. These measures, in addition to the routinely diagnostic procedures, make a broader evaluation of patients" clinical situation and well-being possible. Interventions aimed at improving health care of these patients, such as psychosocial support and/or teaching patients adequate coping strategies should be offered. However, studies are needed to evaluate the effect of these interventions.

Searching for markers to evaluate constitutional symptoms and disease activity is still of benefit. Monitoring of the acute phase response and metabolic changes needs prospective studies to evaluate its usefulness in the follow-up of sarcoidosis patients. Moreover, more research is needed with respect to possible morphologic changes in muscles and muscle energy metabolism in sarcoidosis. In addition, patients with sarcoidosis may profit from rehabilitation programs. In COPD, rehabilitation of patients includes exercise and muscle training, which is effective in increasing exercise tolerance in these patients $[59,60]$. For sarcoidosis patients, research is required for the development of programs and the evaluation of their effect on patients' clinical situation, health status, and QOL. 


\section{References}

1. Neville E, Walker AN, James DG. Prognostic factors predicting the outcome of sarcoidosis: an analysis of 818 patients. QJ Med 1983;208:525-533.

2. Newman LS, Rose CS, Maier LA. Sarcoidosis. N Engl JMed 1997; 336: 12241234.

3. Müller-Quernheim J. The cytokine network in sarcoidosis. Eur Cytokine Netw 1996; 7: 13-26.

4. Sharma OP, Alam S. Diagnosis, pathogenesis, and treatment of sarcoidosis. Curr Opin Pulm Med 1995; 1: 392-400.

5. James DG. Complications of sarcoidosis. Chronic fatigue syndrome. Sarcoidosis 1993; 10: 1-3.

6. Bergner M, Bobbitt RA, Carter WB, Gilson BS. The Sickness Impact Profile: development and final revision of a health status measure. Med Care 1981; 19: 787-805.

7. WHOQOL group. Development of the WHOQOL: rationale and current status. Int J Ment Health 1994; 23: 24-56.

8. Semenzato G, Agostini C. Immunology of sarcoidosis. In: Schwarz MI, King TE, eds. Interstitial Lung Disease. Mosby-Year Book, St. Louis, Missouri 1993; 127158.

9. Lloyd A, Hickie I, Brockman A, Dwyer J, Wakefield D. Cytokine levels in serum and cerebrospinal fluid in patients with chronic fatigue syndrome and control subjects. J Infect Dis 1991; 164: 1023-1024.

10. Arnason BGW. Nervous system-immune system communication. Rev Infect Dis 1991; 13 (suppl 1): S134-137.

11. Elliott MJ, Maini RN, Feldmann M, et al. Randomised double-blind comparison of chimeric monoclonal antibody to tumour necrosis factor $\alpha$ (cA2) versus placebo in rheumatoid arthritis. Lancet 1994; 344: 1105-11 10.

12. Ray C, Weir WRC, Phillips S, Cullen S. Development of a measure of symptoms in chronic fatigue syndrome: The Profile of Fatigue-Related Symptoms (PFRS). Psychol Health 1992; 7: 27-43.

13. Krupp LB, LaRocca NG, Muir-Nash J, Steinberg AD. The Fatigue Severity Scale. Application to patients with multiple sclerosis and systemic lupus erythematosus. Arch Neurol 1989; 46: 1121-1123.

14. De Vries J. Beyond health status. Construction and validation of the Dutch WHO Quality of Life assessment instrument. Doctoral thesis, Tilburg University, Tilburg, The Netherlands, 1996.

15. Van Lankveld W, Näring G, Van der Staak C, Van 't Pad Bosch P, Van de Putte L. Stress caused by rheumatoid arthritis: the relation between subjective stressors of the disease, disease status and well-being. J Behavioral Med 1993; 16:309. 321. 
16. Van Lankveld WGJM. Coping with chronic stressors of rheumatoid arthritis. Doctoral thesis, Catholic University Nijmegen, Nijmegen, The Netherlands, 1993.

17. Meenan RF, Gertman PM, Mason JH, Dunaif R. The Arthritis Impact Measurement Scales: further investigations of health status measure. Arthritis Rheum 1982;25: 1048-1053.

18. Guyatt GH, Berman LB, Townsend M, Pugsley SO, Chambers LW. A measure of quality of life for clinical trials in chronic lung disease. Thorax 1987; 42: 773-778.

19. Jones PW, Quirk FH, Baveystock CM, Littlejohns P. A self-complete measure of health status for chronic airflow limitation: the St. George's Respiratory Questionnaire. Am Rev Respir Dis 1992; 145: 1321-1327.

20. Guyatt GH, Townsend M, Berman LB, Pugsley SO. Quality of life in patients with chronic airflow limitation. Brit J Dis Chest 1987; 81: 45-54.

21. Light RW, Merrill EJ, Despars JA, Gordon GH, Mutalipassi LR. Prevalence of depression and anxiety in patients with COPD. Relationship to functional capacity. Chest 1985; 87: 35-38.

22. Curtis JR, Deyo RA, Hudson LD. Health-related quality of life among patients with chronic obstructive pulmonary disease. Thorax 1994; 49: 162-170.

23. Schrier AC, Dekker FW, Kaptein AA, Dijkman JH. Quality of life in elderly patients with chronic nonspecific lung disease seen in family practice. Chest 1990; 98: 894-899.

24. Jones PW, Baveystock CM, Littlejohns P. Relationships between general health measured with the Sickness Impact Profile and respiratory symptoms, physiological measures, and mood in patients with chronic airflow limitation. Am Rev Respir Dis 1989; 140: 1538-1543.

25. De Vries J, Drent M, Van Heck GL, Wouters EFM. Quality of life in sarcoidosis: a comparison between members of a patient organisation and a random sample. Sarcoidosis Vasc Diffuse Lung Dis 1998; 15: 183-188.

26. KlonoffEA, Kleinhenz ME. Psychological factors in sarcoidosis: the relationship between life stress and pulmonary function. Sarcoidosis 1993; 10:118-124.

27. DeRemee RA. Dysphoria and sarcoidosis. Sarcoidosis Vasc Diffuse Lung Dis 1998; 15:21.

28. Ader R, Felten DL, Cohen N, eds. Psychoneuroimmunology. Academic Press, New York 1991.

29. Keller SE, Shiflett SC, Schleifer SJ, Bartlett JA. Stress, immunity, and health. In: Handbook of Human Stress and Immunity. Glaser $\mathbb{R}$, Kiecolt-Glaser J, eds. Academic Press, San Diego 1994; 217-244.

30. Watkins AD. Hierarchical cortical control of neuroimmunomodulatory pathways. Neuropathol Appl Neurobiol 1994; 20: 423-431.

31. Leonard BE, Song C. Stress and the immune system in the etiology of anxiety and depression. Pharmacol Biochem Behav 1996; 54: 299-303.

32. Smith RS. The macrophage theory of depression. Med Hypoth 1991; 35: 298-306

33. Smith T, Cuzner ML. Neuroendocrine-immune interactions in homeostasis and autoimmunity. Neuropathol Appl Neurobiol 1994; 20:413-421.

34. Heijnen CJ, Kavelaars A. The contribution of neuroendocrine substances to the immune response. Neth J Med 1991; 39: 281-294. 
35. Baydur A, Pandya $\mathrm{K}$, Sharma OP, Kanel GC, Carlson M. Control of ventilation, respiratory muscle strength, and granulomatous involvement of skeletal muscle in patients with sarcoidosis. Chest 1993; 103: 396-402.

36. Badr AI, Sharma OP. Pulmonary function. In: James DG, ed. Sarcoidosis and Other Granulomatous Disorders. New York, Dekker, 1994; 247-266.

37. Dekhuijzen PNR, Gayan-Ramirez G, Decramer M. Does corticosteroid treatment affect the respiratory muscles? Eur Respir J 1993; 6: 465-466.

38. Tobin MJ. Respiratory muscles in disease. Clin Chest Med 1988; 9: 263-286.

39. Killian KJ, Jones NL. Respiratory muscles and dyspnea. Clin Chest Med 1988; 9 : 237-247.

40. Decramer M, Gosselink R, Troosters T, Verschueren M, Evers G. Muscle weakness is related to utilization of health care resources in COPD patients. Etw Respir J 1997; 10: 417-423.

41. Silverstein A, Siltzbach LE. Muscle involvement in sarcoidosis: asymptomatic, myositis, and myopathy. Arch Neurol 1969;21:235-241.

42. Jamal MM, Cilursu AM, Hoffman EL. Sarcoidosis presenting as acute myositis. Report and review of the literature. J Rheumatol 1988; 15: 1868-1871.

43. Stjernberg N, Cajander S, Truedsson H, Uddenfeldt P. Muscle involvement in sarcoidosis. Acta Med Scand 1981; 209:213-216.

44. Landay AL, Jessop C, Lennette ET, Levy JA. Chronic fatigue syndrome: clinical condition associated with immune activation. Lancet 1991; 338: 707-712.

45. Holmes GP, Kaplan JE, Gantz NM, et al. Chronic fatigue syndrome: a working case definition. Ann Intern Med 1988; 108: 387-389.

46. Milner-Brown HS, Miller RG. Muscle strengthening through high-resistance weight training in patients with neuromuscular disorders. Arch Phys Med Rehabil 1988; 69:14-19.

47. McCartney N, Moroz D, Garner SH, McComas AJ. The effects of strength training in patients with selected neuromuscular disorders. Med Sci Sports Exerc 1988; 20: 362-368.

48. Spector SA, Lemmer JT, Koffman BM, et al. Safety and efficacy of strength training in patients with sporadic inclusion body myositis. Muscle Nerve 1997; 20: $1242-1248$.

49. Fagan EA, Dyck RF, Maton PN, et al. Serum levels of C-reactive protein in Crohn's disease and ulcerative colitis. Eur J Clin Invest 1982; 12:351-359.

50. Amos RS, Constable TJ, Crockson RA, Crockson AP, McConkey B. Rheumatoid arthritis: relation of serum $C$-reactive protein and erythrocyte sedimentation rates to radiographic changes. $B r M e d J 1977 ; 1: 195-197$.

51. Young B, Gleeson M, Cripps AW. C-reactive protein: a critical review. Pathology 1991; 23: 118-124.

52. Buck M, Chojkier M. Muscle wasting and dedifferentiation induced by oxidative stress in a murine model of cachexia is prevented by inhibitors of nitric oxide synthesis and antioxidants. EMBOJ 1996; 15: 1753-1765.

53. Fong Y, Moldawer LL, Marano M, et al. Cachectin/TNF or IL-1 $\alpha$ induces cachexia with redistribution of body proteins. Am J Physiol 1989; 256: R659R665. 
54. Beutler B, Cerami A. Cachectin and tumour necrosis factor as two sides of the same biological coin. Nature 1986; 320: 584-588.

55. Schols AMWJ, Buurman WA, Staal-van den Brekel AJ, Dentener MA, Wouters EFM. Evidence for a relation between metabolic derangements and increased levels of inflammatory mediators in a subgroup of patients with chronic obstructive pulmonary disease. Thorax 1996; 51:819-824.

56. Schols AMWJ, Soeters PB, Dingemans AMC, Mostert R, Frantzen PJ, Wouters EFM. Prevalence and characteristics of nutritional depletion in patients with stable COPD eligible for pulmonary rehabilitation. Am Rev Respir Dis 1993; 147: 1151-1156.

57. Pouw EM, Schols AMWJ, Van der Vusse GJ, Wouters EFM. Elevated inosine monophosphate levels in resting muscle of patients with stable COPD. Am J Respir Crit Care Med 1998 (in press).

58. Costabel U, du Bois RM, Eklund A, et.al. Consensus conference: activity of sarcoidosis. Third WASOG meeting, Los Angeles, USA, September 8-11, 1993. Eur Respir J 1994; 7: 624-627.

59. Vale F, Reardon JZ, ZuWallack RL. The long-term benefits of outpatient pulmonary rehabilitation on exercise endurance and quality of life. Chest 1993; 103: $42-45$.

60. Sinclair DJM, Ingram CG. Controlled trial of supervised exercise training in chronic bronchitis. Br Med J 1980; 1: 519-521. 
Chapter 10

\section{Summary \\ Samenvatting \\ Zusammenfassung}




\section{SUMMARY}

Sarcoidosis or Besnier /Boeck's disease is a disorder that can involve virtually any organ of the body. Most frequently it affects the lungs, lymph nodes, skin, and eyes. The histopathological hallmark of sarcoidosis is the non-caseating granuloma, which is found in the organs involved. A granuloma is a rounded structure, which includes a concentration of histiocytes and a variable amount of lymphocytes. The diagnostic procedure of sarcoidosis includes lung radiographs, blood and lung function tests, bronchoalveolar lavage and - when necessary-biopsies. Although the disease has been known for more than 100 years, the cause is still unknown. Therefore, to date, no causal therapy for sarcoidosis is known. When the lung function decreases rapidly, when the eyes, the heart, or the nervous system are affected, or when hypercalcaemia has developed, then treatment with corticosteroids (mostly prednisone) is advised.

The presentation of sarcoidosis is highly variable and the course of the disease is unpredictable. Patients with a sudden onset of the disease are more likely to have a spontaneous remission, whereas in patients with an insidious onset, a chronic course is more probable. The disease can be asymptomatic and accidentally, or not at all, discovered. Symptomatic patients may consult their physician because of symptoms related to the chest such as dyspnea, coughing, and chest pain. Patients may also suffer from nonspecific symptoms such as fatigue, fever, weight loss and muscle pain.

Nonspecific symptoms, such as persistent fatigue, are a major problem for many sarcoidosis patients. These symptoms may remain even when radiographs of the lungs and lung function tests show only slight impairment or have returned to normal. Persistent fatigue may not be taken seriously or may be underestimated by the patient's physician, family, and/or at work. In a number of patients this could result in psychosocial problems.

In a survey among 1026 patient members of the Dutch Sarcoidosis Society fatigue appeared to be the most frequent symptom (chapter 3). Other frequently 
reported symptoms were dyspnea, coughing, joint and muscle pain, as well as reduced exercise capacity and an increased need for sleep. Most of these patients suffered from a chronic course of the disease. Ninety-two percent of them had been ill for more than two years. Most of the symptoms persisted during the course of the disease. Moreover, about one third of the patients suffered from tension, strain, and/or depressive symptoms, which by the patients themselves were attributed to the ongoing disorder.

The impact of disease on patients' lives can be measured by quality of life (QOL) questionnaires. In the present study, QOL was assessed employing the World Health Organization Quality of Life assessment instrument (WHOQOL100) (chapter 5). Sarcoidosis patients showed a lower overall QOL and general health, and suffered from more fatigue and sleeping problems than the healthy control group. This was shown not only in symptomatic sarcoidosis patients, but also in those who reported no actual symptoms at the time of the study. Moreover, patients with current symptoms had problems with their mobility, working capacity, and their activities of daily living compared to healthy control persons as well as asymptomatic sarcoidosis patients. Furthermore, the health status of sarcoidosis patients assessed by the Sickness Impact Profile (SIP), was impaired compared to the health status of healthy controls (chapter $4,7)$, especially in the areas sleep, ability to work, recreation, and social interactions. Furthermore, sarcoidosis patients with bodily symptoms suffered from more depressive symptoms than asymptomatic patients (chapter 4). Rheumatoid arthritis (RA), another chronic disorder, may present with similar symptoms as sarcoidosis, such as joint and muscle pain and fatigue. In about $5 \%$ of the patients studied in the survey among the patient members of the Dutch Sarcoidosis Society, a rheumatic disorder had initially been suggested. Between RA patients and symptomatic sarcoidosis patients no difference was found with regard to fatigue, sleeping problems and impairment of working capacity. However, RA patients suffered from more pain and were more impaired with respect to their mobility (chapter 6). 
As fatigue appeared to be a persistent problem in many sarcoidosis patients, parameters to objectify this symptom are needed. Studies in patients with chronic obstructive pulmonary disease (COPD) have shown that the resting energy expenditure may be increased in these patients. In sarcoidosis patients who suffered from fatigue metabolic derangements were found. Furthermore, decreased respiratory muscle strength was found to be related to symptoms such as fatigue (chapter 7,8).

In conclusion, quality of life (QOL) appeared to be lower in sarcoidosis patients than in healthy persons. With respect to some aspects, QOL of sarcoidosis patients was comparable to the QOL of patients with rheumatoid arthritis. Fatigue was present in a large percentage of sarcoidosis patients. Moreover, sarcoidosis patients suffered from a number of other, often persistent, bodily symptoms. A considerable number of patients reported psychological problems, which were attributed to the disease by the patients. Lung function tests did not correlate well with QOL. However, the increased resting energy expenditure was related to fatigue. Considering the results of the present study, assessment ofQOL should be included in the follow-up and care of sarcoidosis patients. For the individual patient it is crucial that symptoms, including fatigue are taken seriously. In the care of sarcoidosis patients a multi-disciplinary approach is recommended, including somatic as well as psychosocial aspects of this whimsical disorder. 


\section{SAMENVATTING}

Sarcoïdose, ook wel de ziekte van Besnier/Boeck genoemd, is een aandoening, die zich overal in het lichaam kan manifesteren, met name in de longen, lymfeklieren, huid en ogen. Het histopathologische kenmerk van sarcoïdose is het voorkomen van niet-verkazende granulomen in de aangetaste organen. Een granuloom is een min of meer afgeronde structuur bestaande uit een ophoping van histiocytaire cellen met een wisselende hoeveelheid van lymfocytaire cellen. De diagnose wordt bevestigd met behulp van longfoto's, bloed-en longfunctieonderzoek, een bronchoalveolaire lavage (long-spoeling) en indien nodig een biopt (het nemen van stukjes weefsel voor histopathologische analyse). Ondanks het feit dat sarcoïdose reeds meer dan 100 jaar geleden werd beschreven, is de oorzaak van de ziekte nog steeds onbekend. Er is daarom ook geen causale behandeling bekend. Als de longfunctie snel achteruit gaat of andere organen zoals ogen, hart of zenuwstelsel zijn aangetast, of het kalkgehalte in het bloed te hoog is geworden, wordt behandeling met corticosteroïden (meestal prednison) geadviseerd.

De presentatie van sarcoïdose wisselt sterk en het verloop is onvoorspelbaar. Treedt de ziekte plotseling op, dan is de kans groot dat het ziekteproces spontaan geneest. Is het begin echter sluipend, dan is het verloop dikwijls chronisch. De ziekte kan zonder klachten verlopen en dan toevallig of helemaal niet worden ontdekt. Patiënten met klachten melden zich bij de dokter wegens problemen welke mogelijk in verband staan met de longen, zoals kortademigheid, hoesten en/of pijnklachten op de borst. Ook kan er sprake zijn van meer algemene klachten, zoals moeheid, koorts, gewichtsverlies en/of spierpijn.

Aspecifieke klachten - zoals langdurige moeheid - vormen voor veel patiënten met sarcoïdose een groot probleem. Deze klachten kunnen blijven bestaan ondanks de gedeeltelijke of volledige verbetering van de bestaande 
afwijkingen op de longfoto en de longfunctie. De aanhoudende moeheid wordt door de dokter, familie en/of op het werk dikwijls niet ernstig genomen of onderschat. Het niet erkennen van deze moeheidsklachten zou bij een aantal patiënten aanleiding kunnen zijn voor de ontwikkeling van psychosociale problemen.

In een enquête onder 1026 patiënten/leden van de Besnier Boeck/Sarcoïdose Belangenvereniging Nederland (BBSBN) bleek moeheid de meest gerapporteerde klacht te zijn (hoofdstuk 3). Andere frequent genoemde klachten waren kortademigheid, hoesten, gewrichts- en spierpijn, alsmede een verlaagde inspanningscapaciteit en meer behoefte aan slaap. Bij de grote meerderheid van de respondenten was er sprake van een chronisch verloop van de ziekte. Tweëennegentig procent was al langer dan twee jaar ziek. De meeste van de gerapporteerde klachten bleken in de loop der tijd te blijven bestaan of verbeterden maar weinig. Daarnaast had ongeveer een derde van de patiënten last van spanning, stress en/of depressieve symptomen, die door de mensen zelf aan de ziekte werden toegeschreven.

De invloed van ziekte op het leven kan worden onderzocht met behulp van kwaliteit van leven vragenlijsten. Kwaliteit van leven werd met behulp van de World Health Organization Quality of Life assessment instrument (WHOQOL100) gemeten (hoofdstuk 5). Sarcoïdose patiënten hadden een slechtere algemene kwaliteit van leven, minder energie, vaker moeheidsklachten en slaapproblemen vergeleken met de gezonde controlegroep. Ook patiënten, die aangegeven hadden dat zij op het tijdstip van het onderzoek geen klachten hadden, bleken een lagere kwaliteit van leven te hebben dan gezonde controlepersonen. Daarnaast waren de patiënten met actuele klachten beperkt in hun mobiliteit, werkvermogen en dagelijkse levensactiviteiten (ADL) vergeleken met zowel gezonde controlepersonen als asymptomatische patiënten. Verder bleek de gezondheidstoestand, gemeten met de Sickness Impact Profile (SIP), van sarcoïdosepatiënten slechter te zijn dan die van gezonde controlepersonen (hoofdstuk 4,7). Met name slaap, werk, recreatie en 
sociale interacties werden door de ziekte negatief beinvloed. Bij analyse van de depressieve symptomen bleken sarcoidose patiënten met lichamelijke klachten vaker depressieve symptomen te hebben dan patiënten zonder klachten (hoofdstuk 4). Bij reumatoide artritis (RA), een andere chronische ziekte, kunnen evenals bij sarcoïdose symptomen als moeheidsklachten en spier- en gewrichtspijn optreden. Bij ongeveer $5 \%$ van de sarcoïdosepatiënten, die aan de enquête van de BBSBN deelnamen, was oorspronkelijk een vorm van reuma gesuggereerd als oorzaak van de klachten. Tussen RA patiënten en sarcoïdosepatiënten met klachten werd geen verschil gevonden m.b.t. moeheidsklachten, slaapproblemen en vermindering van werkvermogen. Echter, RA patiënten hadden meer pijn en waren meer beperkt in hun mobiliteit (hoofdstuk 6).

Daar moeheid een consistente klacht is bij sarcoïdosepatienten is gezocht naar parameters om die moeheid objectief te meten. Studies bij chronisch obstructief longlijden hebben uitgewezen dat het rustmetabolisme verhoogd kan zijn. Ook bij sarcoïdosepatiënten met actuele moeheidsklachten bleek dit het geval. Een verminderde kracht van de ademhalingsspieren bleek ook gerelateerd aan algemene symptomen, waaronder moeheid (hoofdstuk 7,8).

Samenvattend: de kwaliteit van leven van sarcoïdosepatiënten bleek lager te zijn dan die van gezonde personen en deels vergelijkbaar met die van patiënten met reumatoïde artritis. Moeheid is een veel voorkomende klacht bij sarcoïdosepatiënten. Daarnaast heeft een hoog percentage van de patiënten ook nog andere lichamelijke klachten, die vaak voor langere tijd bleken te blijven bestaan. Een aanzienlijk aantal patiënten rapporteerde psychische problemen. Door de patiënten zelf werden deze psychische problemen aan de sarcoïdose toegeschreven. Longfunctietesten bleken niet gerelateerd te zijn aan kwaliteit van leven. Het verhoogde rustmetabolisme was gerelateerd aan moeheid. Op grond van de bevindingen van dit onderzoek wordt aanbevolen om het meten van de kwaliteit van leven op te nemen als onderdeel bij de begeleiding van sarcoïdosepatiënten. Voor de individuele patiënt is het cruciaal dat de klachten 
inclusief de blijvende moeheid serieus worden genomen. Bij de begeleiding van sarcoildosepatiënten verdient een multi-disciplinaire aanpak - gericht op zowel de lichamelijke als de psychosociale aspecten van deze aandoening - de voorkeur. Alleen dan wordt optimaal aandacht geschonken aan de vele aspecten van deze grillige en vaak onvoorspelbaar verlopende aandoening. 


\section{ZUSAMMENFASSUNG}

Sarkoidose, auch Morbus Besnier/Boeck genannt, ist eine Erkrankung, die sich überall im Körper manifestieren kann, bei der jedoch am häufigsten die Lungen, Lymphknoten, Haut und Augen betroffen sind. Das feingewebliche Merkmal sind Granulome, die in den befallenen Organen vorkommen. Hierbei handelt es sich um mehr oder weniger runde Strukturen, welche aus einer Ansammlung histiozytärer Zellen mit wechselndem Anteil von lymphozytären Zellen bestehen. Die Diagnose wird mit Hilfe von Lungenröntgen, Bluttests, Lungenfunktion, der bronchoalveolären Lavage (Lungenspülung) und, wenn nötig, Biopsien (Gewebeproben) gestellt.

Obwohl die Sarkoidose bereits vor mehr als 100 Jahren beschrieben wurde, ist die Ursache der Erkrankung noch immer unbekannt. Daher steht auch keine kausale Behandlung der Sarkoidose zur Verfügung. Bei schneller Verschlechterung der Lungenfunktion, Beteiligung von Augen, Herz oder Nervensystem, oder bei erhöhtem Kalziumspiegel im Blut, wird eine Behandlung mit Kortison (meist Prednison) empfohlen.

Die Präsentation der Sarkoidose variiert stark, und der Verlauf ist nicht vorhersagbar. Beginnt die Erkrankung akut, dann ist die Chance groß, daß eine spontane Besserung auftritt. Ist der Beginn der Krankheit jedoch schleichend, dann ist der Verlauf oft chronisch. Die Erkrankung kann symptomlos verlaufen und zufällig oder gar nicht entdeckt werden. Patienten mit Beschwerden gehen wegen Symptomen, die mit der Lunge zu tun haben können wie z.B. Husten, Atemnot und/oder Schmerzen im Brustkorb zum Arzt. Es können aber auch unspezifische Symptome wie z.B. Müdigkeit, Fieber, Gewichtsverlust und/ oder Muskelschmerzen auftreten. Diese Allgemeinerscheinungen scheinen für viele Sarkoidosepatienten ein ernstes Problem zu sein. Diese Beschwerden können persistieren, obwohl Lungenröntgen und Lungenfunktion nur noch geringe Veränderungen aufweisen oder wieder normal sind. Wenn die 
Müdigkeit dieser Menschen durch Arzu/Arztin, Familie und/oder am Arbeitsplatz nicht ernst genommen oder unterschätzt werden, könnten zusätzlich zu den körperlichen Beschwerden psychosoziale Probleme entstehen.

Unsere Befragung von 1026 Sarkoidosepatienten, alle Mitglieder der Niederländischen Sarkoidosepatientenvereinigung, zeigte, daß Müdigkeit das am häufigsten genannte Symptom war (Kapitel 3). Außerdem gaben viele Patienten Husten, Kurzatmigkeit, Brustkorbschmerzen, Muskel- und Gelenksschmerzen, sowie schlechte Leistungsfähigkeit und vermehrtes Schlafbedürfnis an. Die Mehrheit dieser Patienten litt an einem chronischen Verlauf der Erkrankung; zweiundneunzig Prozent von ihnen waren schon länger als zwei Jahre krank. Die meisten der angegeben Beschwerden waren über diesen Zeitraum unverändert geblieben oder nur geringfügig besser geworden. Außerdem gab etwa ein Drittel der Patienten psychische Symptome an, die von den Betroffenen selbst mit der Erkrankung in Zusammenhang gebracht wurden.

Die Auswirkungen von Krankheit auf das Leben von Patienten kann mittels Lebensqualitätsfragebögen ermittelt werden. In der vorliegenden Studie wurde die Lebensqualität mit Hilfe des 'World Health Organization Quality of Life assessment instrument" (WHOQOL-100), eines durch die Weltgesundheitsorganisation entwickelten Fragebogens gemessen (Kapitel 5). Sarkoidosepatienten hatten eine schlechtere allgemeine Lebensqualität, weniger Energie, mehr Müdigkeitsbeschwerden und Schlafprobleme als die gesunde Kontrollgruppe. Auch bei Patienten, die zum Zeitpunkt der Studie angegeben hatten, daß sie keine körperlichen Beschwerden hatten, war die Lebensqualität schlechter als die der gesunden Kontrollpersonen. Darüberhinaus waren Patienten mit aktuellen Beschwerden in ihrer Mobilität, ihrem Arbeitsvermögen und ihren täglichen Aktivitäten eingeschränkter als die gesunden Kontrollpersonen und die beschwerdefreien Sarkoidosepatienten. Auch der Gesundheitszustand der Sarkoidosepatienten, gemessen mit dem 'Sickness Impact Profile' (SIP), war 
schlechter als der von Gesunden (Kapitel 4,7). Besonders Schlaf, Arbeit, Erholung, und soziale Interaktionen wurden durch die Erkrankung negativ beeinflußt. Bei der Evaluation von depressiven Symptomen von Sarkoidosepatienten, stellte sich heraus, daß Patienten mit körperlichen Beschwerden öfter depressive Symptome hatten als Patienten ohne körperlichen Beschwerden (Kapitel 4). Bei rheumatoider Arthritis, einer anderen chronischen Erkrankung, können, wie bei Sarkoidose, Symptome wie Müdigkeit, Gelenks- und Muskelschmerzen auftreten. Bei etwa 5\% der Patienten der Umfrage unter den Mitgliedern der Niederländischen Sarkoidosepatientenvereinigung war erst eine rheumatische Erkrankung als Ursache der Beschwerden angenommen worden.Zwischen Patienten mit rheumatoider Arthritis und Sarkoidosepatienten, wurde bzgl. Müdigkeit, Schlafstörungen und Verminderung der Arbeitsfähigkeit kein Unterschied gefunden.Patienten mit rheumatoider Arthritis hatten allerdings mehr Schmerzen und waren in ihrer Beweglichkeit eingeschränkter als die Sarkoidosepatienten (Kapitel 6).

Weil Müdigkeit ein bleibendes Symptom bei Sarkoidosepatienten ist, haben wir nach Parametern gesucht, um dieses Symptom objektiv zu messen. In Studien bei Patienten mit chronisch obstruktiver Lungenerkrankung (COPD) wurde ein erhöhter Ruhestoffwechsel gefunden. Dies war auch der Fall bei Sarkoidosepatienten, die Müdigkeit angaben (Kapitel 8). Patienten bei denen eine Schwäche der Atemmuskulatur festgestellt wurde, litten häufiger unter Müdigkeit (Kapitel 7).

Insgesammt kann gesagt werden, daß die Lebensqualität von Sarkoidosepatienten schlechter ist als die von Gesunden. In einigen Bereichen ist die Lebensqualität symptomatischer Sarkoidosepatienten vergleichbar mit der von Patienten mit rheumatoider Arthritis. Müdigkeit ist ein ernstes Problem für viele Sarkoidosepatienten. Außerdem litt ein hoher Prozentsatz der Patienten an anderen körperlichen Beschwerden, welche über einen längeren Zeitraum bestehen blieben. Eine beträchtliche Anzahl von Patienten gab auch, ebenso der Erkrankung zugeschriebene, psychische Beschwerden an. Die Lungenfunktion 
der Patienten zeigte keinen Zusammenhang zur Lebensqualität. Der erhöhte Ruhestoffwechsel stand in Zusammenhang mit der angegeben Müdigkeit. Aufgrund der vorliegenden Studie scheint uns die routinemäßige Erhebung der Lebensqualität bei Sarkoidosepatienten sinnvoll. Für die einzelnen Patienten ist es essentiell, daß ihre Beschwerden, inklusive der Müdigkeit, ernst genommen werden. Die Behandlung und Begleitung von Sarkoidosepatienten sollte multidisziplinär erfolgen und nicht nur die körperlichen, sondern auch die psychosozialen Probleme miteinbeziehen. 
Abbreviations 


\section{ABBREVIATIONS}
AIMS = Arthritis Impact Measurement Scales
ALS = amyotrophic lateral sclerosis
$\mathrm{BDI}=$ Beck Depression Inventory
$\mathrm{CD}=$ cluster differentiation
$\mathrm{CDI}=$ Cognitive Depression Index
$\mathrm{CFS}=$ chronic fatigue syndrome
$\mathrm{COPD}=$ chronic obstructive pulmonary disease
CNSLD = chronic nonspecific lung disease
$C R P=C$-reactive protein
$\mathrm{CT}=$ computed tomography

DMARD = disease modifying anti-rheumatic drug

EAA = extrinsic allergic alveolitis

$\mathrm{FEV}_{1}=$ forced expiration volume in one second

$\mathrm{FFM}=$ fat-free mass

${ }^{67} \mathrm{Ga}=$ gallium-67 scanning

GM-CFS = granulocyte-macrophage-colony stimulating factor

HRCT $=$ high resolution computed tomography

$\mathrm{IL}=$ interleukin

ILD = interstitial lung disease

INF- $\gamma=$ interferon gamma

$\mathrm{IVC}=$ inspiratory vital capacity

$\mathrm{kgf}=$ kilogram force

$\min =$ minute

NSAID = non steroidal anti-inflammatory drug

$\mathrm{p}=$ probability value

$\mathrm{P}(\mathrm{A}-\mathrm{a}) \mathrm{O}_{2}=$ alveolar-arterial oxygen tension difference

PANAS = Positive Affect Negative Affect Schedule 
PDI = Physical Depression Index

PFRS = Profile of Fatigue-Related Symptoms

$P_{1, \max }=$ maximal inspiratory pressure

$P_{\mathrm{E}, \max }=$ maximal expiratory pressure

pred $=$ predicted

$\mathrm{QOL}=$ quality of life

$\mathrm{RA}=$ rheumatoid arthritis

$\mathrm{REE}=$ resting energy expenditure

$\mathrm{RF}=$ rheumatoid factor

$\mathrm{R} V=$ residual volume

$\mathrm{s}=$ second

$S A C E$ = serum angiotensin converting enzyme

$\mathrm{SD}=$ standard deviation

SIP = Sickness Impact Profile

SPSS = Statistical Package for Social Science

$\mathrm{TH}=\mathrm{T}$ helper

TLC $=$ total lung capacity

$T \mathrm{~L}, \mathrm{CO}=$ transfer factor of the lung for carbon monoxide

TNF $-\alpha=$ tumor necrosis factor alpha

WASOG $=$ World Association of Sarcoidosis and Other Granulomatous

Disorders

WHO = World Health Organization

WHOQOL-100 $=$ World Health Organization Quality of Life assessment instrument

$\mathrm{yr}(\mathrm{s})=\operatorname{year}(\mathrm{s})$ 
Appendix

Questionnaires 


\section{A. 1 Beck Depression Inventory (BDI)}

A. (Mood)

0 I do not feel sad

1 I feel blue or sad

2a I am blue or sad all the time and I can"t snap out of it

$2 b$ I am so sad or unhappy that it is very painful

3 I am so sad or unhappy that I can't stand it

B. (Pessimism)

0 I am not particularly pessimistic or discouraged about the future

1a I feel discouraged about the future

2a I feel I have nothing to look forward to

$2 b$ I feel that I won't ever get over my troubles

3 I feel that the future is hopeless and that things cannot improve

$\mathrm{C}$ (Sense of failure)

0 I do not feel like a failure

1 I feel I have failed more than the average person

2a I feel I have accomplished very little that is worthwhile or that means anything

$2 b$ As I look back on my life all I can see is a lot of failures

3 I feel I am a complete failure as a person (parent, husband, wife)

D (Lack of Satisfaction)

0 I am not particularly dissatisfied

la I feel bored most of the time

1b I don't enjoy things the way I used to

2 I don't get satisfaction out of anything any more

3 I am dissatisfied with everything

E (Guilty Feeling)

0 I don't feel particularly guilty

1 I feel bad or unworthy a good part of the time

2 a I feel quite guilty

$2 \mathrm{~b}$ I feel bad or unworthy practically all the time now

3 I feel as though I am very bad or worthless 
$F$ (Sense of Punishment)

0 I don't feel I am being punished

1 I have a feeling that something bad may happen to me

2 I feel I am being punished or will be punished

3a I feel I deserve to be punished

$3 \mathrm{~b}$ I want to be punished

G(Self Hate)

0 I don't feel disappointed in myself

I a I am disappointed in myself

Ib I don't like myself

2 I am disgusted with myself

3 I hate myself

H (Self Accusations)

0 I don't feel I am any worse than anybody ellse

1 I am very critical of myself for my weaknesses or mistakes

2a I blame myself for everything that goes wrong

2b I feel I have many bad faults

I (Sellf-punitive Wishes)

0 I don't have any thoughts of harming myself

1 I have thoughts of harming myself but I would not carry them out

$2 \mathrm{a}$ I feel I would be better off dead

$2 \mathrm{~b}$ I have definite plans about committing suicide

2c I feel my family would be better off if I were dead

3 I would kill myself if I could

$J$ (Crying Spells)

0 I don't cry any more than usual

1 I cry more now than'I used to

2 I cry all the time now. I can't stop it

3 I used to be able to cry but now I can't cry at all even though I want to

K (Irritability)

0 I am no more irritated now than I ever am

1 I get annoyed or irritated more easily than I used to

2 I feel irritated all the time

3 I don't get irritated at all at the things that used to irritate me 
L. (Social Withdrawal)

0 I have not lost interest in other people

1. I am less interested in other people now than I used to be

2 I have lost most of my interest in other people and have little feeling for them

3 I have lost all my interest in other people and don't care about them at all

M(Indecisiveness)

0 I make decisions about as well as ever

I I am less sure of myself now and try to put off making decisions

2 I can't make decisions any more without help

3 I can't make any decisions at all any more

N (Body Image)

0 I don't feel I look any worse than I used to

1 I am worried that I am looking old or unattractive

2 I feel that there are permanent changes in my appearance and they make me look unattractive

3 I feel that I am ugly of repulsive looking

$O$ (Work Inhibition)

$0 \quad$ I can work about as well as before

la It takes extra effort to get started at doing something

1b I don't work as well as I used to

2 I have to push myself very hard to do anything

3 I can't do any work at all

P (Sleep Disturbances)

0 I can sleep as well as usual

1 I wake up more tired in the moming than I used to

2 I wake up 1-2 hours earlier than usual and find it hard to get back to sleep

3 I wake up early every day and can't get more than 5 hours sleep

Q (Fatigability)

0 I don't get any more tired than usual

I I get tired more easily than I used to

2 I get tired from doing anything

3 I get too tired to do anything 
$\mathrm{R}$ (Loss of Appetite)

0 My appetite is no worse than usual

1 My appetite is not as good as it used to be

2 My appetite is much worse now

3 I have no appetite at all any more

S (Weight Loss)

0 I haven't lost much weight, if any, lately

1 I have lost more than 5 pounds

2 I have lost more than 10 pounds

3 I have lost more than 15 pounds

T (Somatic Preoccupation)

0 I am no more concerned about my health than usual

1 I am concerned about aches and pains or upset stomach or constipation or other unpleasant feelings in my body

2 I an so concerned with how I feel or what I feel that it's hard to think of much else

3. I am completely abosrbed in what I feel

U (Loss of Libido)

0 I have not noticed any recent change in my interest in sex

1 I am less interested in sex than I used to be

2 I am much less interested in sex now

3 I have lost interest in sex completely

Beck AT, Ward $\mathrm{CH}$, Mendelson $\mathrm{M}$, Mock J, Erbaugh J. An Inventory for Measuring Depression. Arch Gen Psychiatry 1961; 4: 561-571. 


\section{A. 2 Positive Affect Negative Affect Schedule (PANAS)}

This scale consists of a number of words that describe different feelings and emotions. Read each item and then mark the appropriate answer in the space next to that word. Indicate to what extent you generally feel this way, that is, how you feel on the average. Use the following scale to record your answers.

1

very slightly or

not at all
2

a little

not at all.
3

4

moderately quite a bit
5

extremely

$\begin{array}{llllll}\text { interested } & 1 & 2 & 3 & 4 & 5 \\ \text { distressed } & 1 & 2 & 3 & 4 & 5 \\ \text { excited } & 1 & 2 & 3 & 4 & 5 \\ \text { upset } & 1 & 2 & 3 & 4 & 5 \\ \text { strong } & 1 & 2 & 3 & 4 & 5 \\ \text { guilty } & 1 & 2 & 3 & 4 & 5 \\ \text { scared } & 1 & 2 & 3 & 4 & 5 \\ \text { hostile } & 1 & 2 & 3 & 4 & 5 \\ \text { enthusiastic } & 1 & 2 & 3 & 4 & 5 \\ \text { proud } & 1 & 2 & 3 & 4 & 5 \\ \text { irritable } & 1 & 2 & 3 & 4 & 5 \\ \text { alert } & 1 & 2 & 3 & 4 & 5 \\ \text { ashamed } & 1 & 2 & 3 & 4 & 5 \\ \text { inspired } & 1 & 2 & 3 & 4 & 5 \\ \text { nervous } & 1 & 2 & 3 & 4 & 5 \\ \text { determined } & 1 & 2 & 3 & 4 & 5 \\ \text { attentive } & 1 & 2 & 3 & 4 & 5 \\ \text { jittery } & 1 & 2 & 3 & 4 & 5 \\ \text { active } & 1 & 2 & 3 & 4 & 5 \\ \text { afraid } & 1 & 2 & 3 & 4 & 5\end{array}$

Watson D, Clark LA, Tellegen A. Development and validation of brief measures of positive and negative affect: the PANAS scales. J Pers Soc Psych 1988; 54: 1063-1070. 


\section{A. 3 Sickness Impact Profile (SIP)}

PLEASE RESPOND TO (CHECK) ONLY THOSE STATEMENTS THAT YOU ARE SURE DESCRIBE YOU TODAY AND ARE RELATED TO YOUR STATE OF HEALTH.

1. I spend much of the day lying down in order to rest

2. I sit during much of the day

3. I am sleeping or dozing most of the time - day and night

4. I lie down more often during the day in order to rest

5. I sit around half-asleep

I sleep less at night, for example, wake up too early, don't fall asleep for a long time, awaken frequently

7. I sleep or nap more during the day

PLEASE RESPOND TO (CHECK) ONLY THOSE STATEMENTS THAT YOU ARE SURE DESCRIBE YOU TODAY AND ARE RELATED TO YOUR STATE OF HEALTH.

1. I say how bad or useless I am, for example, that I am a burden on orders

2. I laugh or cry suddenly

3. I often moan and groan in pain or discomfort

4. I have attempted suidice

5. I act nervous or restless

6. I keep rubbing or holding areas of my body that hurt or are uncomfortable

7. I act irritable and impatient with myself, for example, talk badly about myself, swear at myself, blame myself for things that happen

8. I talk about the future in a hopeless way

9. I get sudden frights

PLEASE RESPOND TO (CHECK) ONLY THOSE STATEMENTS THAT YOU ARE SURE DESCRIBE YOU TODAY AND ARE RELATED TO YOUR STATE OF HEALTH. 
1. I make difficult moves with help, for example, getting into or out of cars, bathtubs

2. I do not move into or out of bed or chair by myself but am moved by a person or mechanical aid

3. I stand only for short periods of time

4. I do not maintain balance

5. I move my hands or fingers with some limitation or difficulty

6. I stand up only with someone"s help

7. I kneel, stoop, or bend down only by holding on to something

8. I am in a restricted position all the time

9. I am very clumsy in body movements

10. I get in and out of bed or chairs by grasping something for support or using a cane or walker

11. I stay lying down most of the time

12. I change position frequently

13. I hold on to something to move myself around in bed

14. I do not bathe myself completely, for example, require assistance with bathing

15. I do not bathe myself at all, but am bathed by someone else

16. I use bedpan with assistance

17. I have trouble getting shoes, socks, or stockings on

18. I do not have control of my bladder

19. I do not fasten my clothing, for example, require assistance with buttons, zippers, shoelaces

20. I spend most of the time partly undressed or in pajamas

21. I do not have control of my bowels

22. I dress myself, but do so very slowly

23. I get dressed only with someone's help

THIS GROUP OF STATEMENTS HAS TO DO WITH ANY WORK YOU USUALLY DO IN CARING FOR YOUR HOME OR YARD. CONSIDERING JUST THOSE THINGS THAT YOU DO, PLEASE RESPOND TO (CHECK) ONLY THOSE STATEMENTS THAT YOU ARE SURE DESCRIBE YOU TODAY AND ARE RELATED TO YOUR STATE OF HEALTH

\footnotetext{
1. I dow work around the house only for short periods of time or rest often
} 
2. I am doing less of the regular daily work around the house than I would usually do

3. I am not doing any of the regular daily work around the house than I would usually do

4. I am not doing any of the maintenance or repair work that I would usually do in my home or yard

5. I am not doing any of the shopping that I would usually do

6. I am not doing any of the house cleaning that I would usually do

7. I have difficulty doing handwork, for example, turning faucets, using kitchen gadgets, sewing, carpentry

8. I am not doing any of the clothes washing that I would usually do

9. I am not doing heavy work around the house

10. I have given up taking care of personal or household business affairs, for example, paying bills, banking, working on budget

PLEASE RESPOND TO (CHECK) ONLY THOSE STATEMENTS THAT YOU ARE SURE DESCRIBE YOU TODAY AND ARE RELATED TO YOUR STATE OF HEALTH.

1. I am getting around only within one building

2. I stay within one room

3. I am staying in bed more

4. I am staying in bed most of the time

5. I am not now using public transportation

6. I stay home most of the time

7. I am only going to places with restrooms nearby

8. I am not going into town

9. I stay away from home only for brief periods of time

10. I do not get around in the dark or in unlit places without someone's help

PLEASE RESPOND TO (CHECK) ONLY THOSE STATEMENTS THAT YOU ARE SURE DESCRIBE YOU TODAY AND ARE RELATED TO YOUR STATE OF HEALTH. 
1. I am going out less to visit people

2. I am not going out to visit people at all

3. I show less interest in other people's problems, for example, don't listen when they tell me about their problems, don't offer to help

4. I often act irritable toward those around me, for example, snap at people, give sharp answers, criticize easily

5. I show less affection

6. I am doing fewer social activities with groups of people

7. I am cutting down the length of visits with friends

8. I am avoiding social visits from others

9. My sexual activity is decreased

10. I often express concern over what might be happening to my health

11. I talk less with those around me

12. I make many demands, for example, insist that people do things for me, tell them how to do things

13. I stay alone much of the time

14. I act disagreeable to family members, for example, I act spiteful, I am stubborn

15. T have frequent outbursts of anger at family members, for example, strike at them, scream, throw things at them

16. I isolate myself as much as I can from the rest of the family

17. I am paying less attention to the children

18. I refuse contact with family members, for example, turn away form them

19. I am not doing the things I usually do to take care of my children of family

20. I am not joking with family members as I usually do

PLEASE RESPOND TO (CHECK) ONLY THOSE STATEMENTS THAT YOU ARE SURE DESCRIBE YOU TODAY AND ARE RELATED TO YOUR STATE OF HEALTH.

1. I walk shorter distances or stop to rest often

2. I do not walk up or down hills

3. I use stairs only with mechanical support, for example, handrail, cane, crutches 
4. I walk up or down stairs only with assistance from someone else

5. I get around in a wheelchair

6. I do not walk at all

7. I walk by myself but with some difficulty, for example, limp, wobble, stumble, have stiff leg

8. I walk only with help from someone

9. I go up and down stairs more slowly, for example, one step at a time, stop often

10. I do not use stairs at all

11. I get around only by using a walker, crutches, cane, walls, or furniture

12. I walk more slowly

PLEASE RESPOND TO (CHECK) ONLY THOSE STATEMENTS THAT YOU ARE SURE DESCRIBE YOU TODAY AND ARE RELATED TO YOUR STATE OF HEALTH.

1. I am confused and start several actions at a time

2. Thave more minor accidents, for example, drop things trip and fall, bump into things

3. I react slowly to things that are said or done

4. I do not finish things I start

5. I have difficulty reasoning and solving problems, for example, making plans, making decisions, learning new things

6. I sometimes behave as if I were confused or disoriented in place or time, for example, where I am, who is around, directions, what day it is

7. I forget a lot, for example, things that happened recentlly, where I put things, appointments

8. I do not keep my attention on any activity for long

9. I make more mistakes than usual

10. I have difficulty doing activities involving concentration and thinking 
PLEASE RESPOND TO (CHECK) ONLY THOSE STATEMENTS THAT YOU ARE SURE DESCRIBE YOU TODAY AND ARE RELATED TO YOUR STATE OF HEALTH.

1. I am having trouble writing or typing

2. I communicate mostly by gestures, for example, moving head, pointing, sign language

3. My speech is understood only by a few people who know me well

4. I often lose control of my voice when I talk, for example, my voice gets louder or softer, trembles, changes unexpectedly

5. I don't wirte except to sign my name

6. I carry on a conversation only when very close to the other person or looking at him

7. I have difficulty speaking, for example, get stuck, stutter, stammer, slur my words

8. I am understood with difficulty

9. I do not speak clearly when I am under stress

THE NEXT GROUP OF STATEMENTS HAS TO DO WITH ANY WORK YOU USUALLY DO OTHER THAN MANAGING YOUR HOME. BY THIS WE MEAN ANYTHING THAT YOUREGARD AS WORK THAT YOU DO ON A REGULAR BASIS.

DO YOU USUALLY DO WORK OTHER THAN

MANAGING YOUR HOME?

YES NO

IF YOU ANSWERED YES, GO ON TO THE NEXT PAGE.

IF YOU ANSWERED NO:

ARE YOU RETIRED?

YES NO

IF YOU ARE RETIRED, WAS YOUR RETIREMENT RELATED TO YOUR HEALTH?

YES NO 
IF YOU ARE NOT RETIRED, BUT ARE NOT WORKING, IS THIS RELATED TO YOUR HEALTH?

YES NO

IF YOU ARE NOT WORKING AND IT IS NOT BECAUSE OF YOUR HEALTH, PLEASE SKIP THIS PAGE.

NOW CONSIDER THE WORK YOU DO AND RESPOND TO (CHECK) ONLY THOSE STATEMENTS THAT YOU ARE SURE DESCRIBE YOU TODAY AND ARE RELATED TO YOUR STATE OF HEALTH. (IF TODAY IS A SATURDAY OR SUNDAY OR SOME OTHER DAY THAT YOU WOULD USUALLY HAVE OFF, PLEASE RESPOND AS IF TODAY WERE A WORKING DAY.)

1. I am not working at all

(IF YOU CHECKED THIS STATEMENT, SKIP TO THE NEXT PAGE.)

2. I am doing part of my job at home

3. I am not accomplishing as much as usual at work

4. I often act irritable toward my work associates, for example, snap at them, give sharp answers, criticize easily

5. I am working shorter hours

6. I am doing only light work

7. I work only for short periods of time or take frequent rests

8. I am working at my usual job but with some changes, for example, using different tools or special aids, trading some tasks with other workers

9. I do not do my job as carefully and accurately as usual

THIS GROUP OF STATEMENTS HAS TO DO WTTH ACTIVITIES YOU USUALLY DO IN YOUR FREE TIME. THESE ACTIVITIES ARE THINGS THAT YOU MIGHT DO FOR RELAXATION, TO PASS THE TIME, OR FOR ENTERTAINMENT. PLEASE RESPOND TO (CHECK) ONLY THOSE STATEMENTS THAT YOU ARE SURE DESCRIBE YOU TODAY AND ARE RELATED TO YOUR STATE OF HEALTH.

1. I do my hobbies and recreation for shorter periods of time 
2. I am going out for entertainment less often

3. I am cutting down on some of my usual inactive recreation and pastimes, for example, watching TV, playing cards, reading

4. I am not doing any of my usual inactive recreation and pastimes, for example, watching TV, playing cards, reading

5. I am doing more inactive pastimes in place of my other usual activities

6. I am doing fewer community activities

7. I am cutting down on some of my usual physical recreation or activities

8. I am not doing any of my usual physical recreation or activities

PLEASE RESPOND TO (CHECK) ONLY THOSE STATEMENTS THAT YOU ARE SURE DESCRIBE YOU TODAY AND ARE RELATED TO YOUR STATE OF HEALTH.

1. I am eating much less than usual

2. If feed myself but only by using specially prepared food or utensils

3. I am eating special or different food, for example, sorft food, bland diet, low-salt, low-fat, low-sugar

4. I eat no food at all but am taking fluids

5. I just pick or nibble at my food

6. I am drinking less fluids

7. If feed myself with help from someone else

8. I do not feed myself at all, but must be fed

9. I am eating no food at all, nutrition is taken through tubes or intravenous fluids

Bergner M, Bobbit RA, Carter WB, Gilson BS. The Sickness Impact Profile: development and final revision of a health status measure. Med Care 1981; 19: 787-805. 


\section{A. 4 World Health Organization of Quality of Life assessment instrument 100 (WHOQOL-100)}

Instructions

This questionnaire asks how you feel about your quality of life, health, and other areas of your life. Please answer all the questions. If you are unsure about which response to give to a question, please choose the one that appears most appropriate. This can often be your first response.

Please keep in mind your standards, hopes, pleasures and concerns. We ask that you think about your life in the last two weeks.

For example, thinking about the last two weeks, a question might ask:

How much do you worry about your health?

\begin{tabular}{c|c|c|c|c} 
Not at all & A litule & A moderate amount & Very much & An extreme amount \\
1 & 2 & 3 & 4 & 5
\end{tabular}

You should circle the number that best fits how much you have worried about your health over the last two weeks. So you would circle the number 4 if you worried about your health "Very much", or circle number 1 if you have worried "Not at all" about your health. Please read each question, assess your feelings, and circle the number on the scale for each question that gives the best answer for you.

Thank you for your help

The following questions ask about how much you have experienced certain things in the last two weeks, for example, positive feelings such as happiness or contentment. If you have experienced these things an extreme amount circle the number next to "An extreme amount". If you have not experienced these things at all, circle the number next to "Not at all". You should circle one of the numbers in between if you wish to indicate your answer lies somewhere between "Not at all." and "Extremely". Questions refer to the last two weeks.

F1.2 Do you worry about your pain or discomfort?

\begin{tabular}{c|c|c|c|c} 
Not at all & A little & A moderate amount & Very much & An extreme amount \\
1 & 2 & 3 & 4 & 5
\end{tabular}


F1.3 How difficult is it for you to handle any pain or discomfort?

\begin{tabular}{c|c|c|c|c} 
Not at all & slightly & Moderately & Very & Extremely \\
1 & 2 & 3 & 4 & 5
\end{tabular}

F1.4 To what extent do you feel that (physical) pain prevents you from doing what you need to do?

\begin{tabular}{c|c|c|c|c} 
Not at all & A little & A moderate amount & Very much & An extreme amount \\
1 & 2 & 3 & 4 & 5
\end{tabular}

F2.1 How easily do you get tired?

\begin{tabular}{c|c|c|c|c} 
Not at all & Slightly & Moderately & Very & Extremely \\
1 & 2 & 3 & 4 & 5
\end{tabular}

F2.4 How much are you bothered by fatigue?

\begin{tabular}{c|c|c|c|c} 
Not at all & Slightly & Moderately & Very & Extremely \\
1 & 2 & 3 & 4 & 5
\end{tabular}

F3.2 Do you have any difficulties with sleeping?

\begin{tabular}{c|c|c|c|c} 
None at all & A little & A moderate amount & Very much & An extreme amount \\
1 & 2 & 3 & 4 & 5
\end{tabular}

F3.4 How much do any sleep problems worry you?

\begin{tabular}{c|c|c|c|c} 
Not at all & A little & A moderate amount & Very much & An extreme amount \\
1 & 2 & 3 & 4 & 5
\end{tabular}

F4. 1 How much do you enjoy life?

\begin{tabular}{c|c|c|c|c} 
Not at all & A little & A moderate amount & Very much & An extreme amount \\
1 & 2 & 3 & 4 & 5
\end{tabular}

F4.3 How positive do you feel about the future?

\begin{tabular}{c|c|c|c|c} 
Not at all & Slitghly & Moderately & Very & Extremely \\
1. & 2 & 3 & 4 & 5
\end{tabular}

F4.4 How much do you experience positive feelings in your life?
Not at all
A litile
A moderate amount
Very much
An extreme amount
3
4
5 
F5.2 How well are you able to concentrate?

\begin{tabular}{c|c|c|c|c} 
Not at all & Slightly & Moderately & Very well & Extremely \\
1 & 2 & 3 & 4 & 5
\end{tabular}

F6. 1 How much do you value yourself?
Not at all
A little
A moderate amount
Very much
4
An extreme amount
2
3
5

F6.2 How much confidence do you have in yourself?

\begin{tabular}{c|c|c|c|c} 
Not at all & A little & A moderate amount & Very much & An extreme amount \\
1 & 2 & 3 & 4 & 5
\end{tabular}

F7. 1 Do you feel inhibited by your looks?
Not at all
Slightly
Moderately
3
Very much
Extremely
5

F7.3 Is there any part of your appearance which makes you feel uncomfortable?

\begin{tabular}{c||c|c|c|c} 
Not at all & A little & A moderate amount & Very much & An extreme amount \\
1 & 2 & 3 & 4 & 5
\end{tabular}

F8.2 How worried do you feel?
\begin{tabular}{c||c|} 
Not at all & Slightly \\
1 & 2
\end{tabular}
Moderately
3
Very
4
Extremely
5

F8.3 How much do any feelings of sadness or depression interfere with your everyday functuroning?

Not at all
A litule
A moderate amount
2
3
2
.

Very much
4

An extreme amount

1

5

F8.4 How much do any feelings of depression bother you?

Not at all

1
A little

2
A moderate amount

3
Very much 4
An extreme amount
5

F10.2 To what extent do you have difficulty in performing your routine activities?

Not at all

A litule

A moderate amount

Very much

An extreme amount

3

4

5 
F10.4 How much are you bothered by any limitations in performing everyday living activities?

\begin{tabular}{c|c|c|c|c} 
Not at all & A litule & A moderate amount & Very much & An extreme amount \\
1 & 2 & 3 & 4 & 5
\end{tabular}

F11.2 How much do you need any medication to function in your dailly life?

\begin{tabular}{c|c|c||c|c} 
Not at all & A little & A moderate amount & Very much & An extreme amount \\
1 & 2 & 3 & 4 & 5
\end{tabular}

F11.3 How much do you need any medical treatment to function in your daily life?

\begin{tabular}{c|c|c|c|c} 
Not at all & A little & A moderate amount & Very much & An extreme arnount \\
1 & 2 & 3 & 4 & 5
\end{tabular}

F11.4 To what extent does your quality of life depend on the use of medical substances or medical aids?

\begin{tabular}{c|c|c|c|c} 
Not at all & A little & A moderate amount & Very much & An extreme amount \\
1 & 2 & 3 & 4 & 5
\end{tabular}

F13.1 How alone do you feel in your life?

\begin{tabular}{c|c|c|c|c} 
Not at all & Slightly & Moderately & Very much & Extremely \\
1 & 2 & 3 & 4 & 5
\end{tabular}

F15.2 How well are your sexual needs fulfilled?

\begin{tabular}{c|c|c|c|c} 
Not at all & Slightly & Moderately & Very much & Extremely \\
1 & 2 & 3 & 4 & 5
\end{tabular}

F15.4 Are you bothered by any difficulties in your sex life?

\begin{tabular}{c|c|c|c|c} 
Not at all & Slightly & Moderately & Very & Extremely \\
1 & 2 & 3 & 4 & 5
\end{tabular}

F16.1 How safe do you feel in your daily life?

\begin{tabular}{c|c|c|c|c} 
Not at all & Slightly & Moderately & Very & Extremely \\
1 & 2 & 3 & 4 & 5
\end{tabular}

F16.2 Do you feel you are living in a safe and secure environment?

\begin{tabular}{c|c|c|c|c} 
Not at all & Slightly & Moderately & Very much & Extremely \\
1 & 2 & 3 & 4 & 5
\end{tabular}


F16.3 How much do you worry about your safety and security?

\begin{tabular}{c|c|c|c|c} 
Not at all & A little & A moderate amount & Very much & An extreme amount \\
1 & 2 & 3 & 4 & 5
\end{tabular}

F17.1 How comfortable is the place where you live?

\begin{tabular}{c|c|c|c|c} 
Not at all & Slightly & Moderately & Very & Extremely \\
1 & 2 & 3 & 4 & 5
\end{tabular}

F17.4 How much do you like it where you live?

\begin{tabular}{c|c|c|c|c} 
Not at all & A little & A moderate amount & Very much & An extreme amount \\
1 & 2 & 3 & 4 & 5
\end{tabular}

F18.2 Do you have financial difficulties?

\begin{tabular}{c|c|c|c|c} 
Not at all & A little & A moderate amount & Very much & An extreme amount \\
1 & 2 & 3 & 4 & 5
\end{tabular}

F18.4 How much do you worry about money?

\begin{tabular}{c|c|c|c|c} 
Not at all & A little & A moderate amount & Very much & An extreme amount \\
1 & 2 & 3 & 4 & 5
\end{tabular}

F19.1 How easily are you able to get good medical care?

\begin{tabular}{c||c|c|c|c} 
Not at all & Slightly & Moderately & Very & Extremely \\
1 & 2 & 3 & 4 & 5
\end{tabular}

F21.3 How much do you enjoy your free time?

\begin{tabular}{c|c|c|c|c} 
Not at all & A little & Moderately & Very much & An extreme amount \\
1 & 2 & 3 & 4 & 5
\end{tabular}

122.1 How healthy is your physical environment?

\begin{tabular}{c|c|c|c|c} 
Not at all & Slightly & Moderately & very & Extremely \\
1 & 2 & 3 & 4 & 5
\end{tabular}

F22.2 How concerned are you with the noise in the area you live in?

\begin{tabular}{c|c|c|c|c} 
Not at all & A little & Moderately & Very much & An extreme amount \\
1 & 2 & 3 & 4 & 5
\end{tabular} 
F23.2 To what extent do you have problems with transport?

\begin{tabular}{c|c|c|c|c} 
Not at all & A little & A moderate amount & Very much & An extreme amount \\
1 & 2 & 3 & 4 & 5
\end{tabular}

F23.4 How much do difficulties with transport restrict your life?

\begin{tabular}{c|c|c|c|c} 
Not at all & A little & A moderate amount & Very much & An extreme amount \\
1 & 2 & 3 & 4 & 5
\end{tabular}

The following questions ask about how completely you experience or were able to do certain things in the last two weeks, for example activities of daily living such as washing. dressing or eating. If you have been able to do these things completely, circle the number next to "Completely". If you have not been able to do these things at all, circle the number next to "Not at all". You should circle one of the numbers in between if you wish to indicate your answer lies somewhere between "Not at all" and "Completely". Questions refer to the last two weeks.

F2.1 Do you have enough energy for everyday life?

\begin{tabular}{c|c|c|c|c} 
Not at all & A little & Moderately & Mosily & Completely \\
1 & 2 & 3 & 4 & 5
\end{tabular}

F7. 1 Are you able to accept your bodily appearance?

\begin{tabular}{c|c|c|c|c} 
Not at all & A little & Moderately & Mostly & Completely \\
1 & 2 & 3 & 4 & 5
\end{tabular}

F10.1 To what extent are you able to carry out your daily activities? \begin{tabular}{c|c|c|c|c} 
Not at all & A little & Moderately & Mostly & Completely \\
1 & 2 & 3 & 4 & 5
\end{tabular}

F11.1 How dependent are you on medications?

\begin{tabular}{c|c|c|c|c} 
Not at all & A litte & Moderately & Mostly & Completely \\
1 & 2 & 3 & 4 & 5
\end{tabular}

F14.1 Do you get the kind of support from others that you need?

\begin{tabular}{c|c|c|c|c} 
Not at all & A little & Moderately & Mostly & Completely \\
1 & 2 & 3 & 4 & 5
\end{tabular}


F14.2 To what extent can you count on your friends when you need them?

\begin{tabular}{c|c|c|c|c} 
Not at all & A little & Moderately & Mostly & Completely \\
1 & 2 & 3 & 4 & 5
\end{tabular}

F17.2 To what degree does the quality of your home meet your needs?

\begin{tabular}{c|c|c|c|c} 
Not at all & A little & Moderately & Mostly & Completely \\
1 & 2 & 3 & 4 & 5
\end{tabular}

F18.1 Have you enough money to meet your needs?

\begin{tabular}{c|c|c|c|c} 
Not at all & A little & Moderately & Mostly & Completely \\
1 & 2 & 3 & 4 & 5
\end{tabular}

F20.1 How available to you is the information that you need in your day-to-day life?
Not at all
A little
Moderately
3
Mostly
4
Completely
5

F20.2 To what extent do you have opportunities for acquiring the information that you feel you need?

\begin{tabular}{c|c|c|c|c} 
Not at all & A little & Moderately & Mostly & Completely \\
1 & 2 & 3 & 4 & 5
\end{tabular}

F21.1 To what extent do you have the opportunity for leisure activities?

\begin{tabular}{c|c|c|c|c} 
Not at all & A little & Moderately & Mostly & Completely \\
1 & 2 & 3 & 4 & 5
\end{tabular}

F21.2 How much are you able to relax and enjoy yourself?

\begin{tabular}{c|c|c|c|c} 
Not at all & A little & Moderately & Mostly & Completely \\
1 & 2 & 3 & 4 & 5
\end{tabular}

F23.1 To what extent do you have adequate means of transport?
Not at all

\begin{tabular}{c|c} 
A litile \\
2
\end{tabular}
Moderately
3
Mostly
Completely

The following questions ask you to say how satisfied, happy or good you have felt about various aspects of your life over the last two weeks. For example, about your family life or the energy that you have. Decide how satisfied or dissatisfied you are with each aspect of your life and circle the number that best fits how you feel about this. Questions refer to the last two weeks. 
G.2 How satisfied are you with the quality of your life?

\begin{tabular}{c|c|c|c|c}
$\begin{array}{c}\text { Very dissa- } \\
\text { tisfied }\end{array}$ & $\begin{array}{c}\text { Dissatis- } \\
\text { fied }\end{array}$ & $\begin{array}{c}\text { Neither satisfied nor } \\
\text { dissatisfied }\end{array}$ & Satisfied & Very satisfied \\
1 & 2 & 3 & 4 & 5
\end{tabular}

G3 In general, how satisfied are you with your life?

\begin{tabular}{c|c|c}
$\begin{array}{c}\text { Very dissa- } \\
\text { tisfied }\end{array}$ & Dissatis- & $\begin{array}{c}\text { Neither satisfied nor } \\
\text { fied }\end{array}$ \\
1 & 2 & dissatisfied
\end{tabular}

Satisfied

Very satisfied

5

G4 How satisfied are you with your health?

\begin{tabular}{c|c|c|c|c}
$\begin{array}{c}\text { Very dissa- } \\
\text { tisfied }\end{array}$ & $\begin{array}{c}\text { Dissatis- } \\
\text { fied }\end{array}$ & $\begin{array}{c}\text { Neither satisfied nor } \\
\text { dissatisfied }\end{array}$ & Satisfied & Very satisfied \\
1 & 2 & 3 & 4 & 5
\end{tabular}

F.2.3 How satisfied are you with the energy that you have?

\begin{tabular}{c|c|c|c|c}
$\begin{array}{c}\text { Very dissa- } \\
\text { tisfied }\end{array}$ & $\begin{array}{c}\text { Dissatis- } \\
\text { fied }\end{array}$ & $\begin{array}{c}\text { Neither satisfied nor } \\
\text { dissatisfied }\end{array}$ & Satisfied & Very satisfied \\
1 & 2 & 3 & 4 & 5
\end{tabular}

F3.3 How satisfied are you with your sleep?

\begin{tabular}{c|c|c|c|c}
$\begin{array}{c}\text { Very dissa- } \\
\text { tisfied }\end{array}$ & $\begin{array}{c}\text { Dissatis- } \\
\text { fied }\end{array}$ & $\begin{array}{c}\text { Neither satisfied nor } \\
\text { dissatisfied }\end{array}$ & Satisfied & Very satisfied \\
1 & 2 & 3 & 4 & 5
\end{tabular}

F5.2 How satisfied are you with your ability to learn new information?

\begin{tabular}{c|c|c|c|c}
$\begin{array}{c}\text { Very dissa- } \\
\text { tisfied }\end{array}$ & $\begin{array}{c}\text { Dissatis- } \\
\text { fied }\end{array}$ & $\begin{array}{c}\text { Neither satisfied nor } \\
\text { dissatisfied }\end{array}$ & Satisfied & Very satisfied \\
1. & 2 & 3 & 4 & 5
\end{tabular}

F5.4 How satisfied are you with your ability to make decisions?

\begin{tabular}{c||c|c|c|c}
$\begin{array}{c}\text { Very dissa- } \\
\text { tisfied }\end{array}$ & $\begin{array}{c}\text { Dissatis- } \\
\text { fied }\end{array}$ & $\begin{array}{c}\text { Neither satisfied nor } \\
\text { dissatisfied }\end{array}$ & Satisfied & Very satisfied \\
1 & 2 & 3 & 4 & 5
\end{tabular}


F6.3 How satisfied are you with yourself?

\begin{tabular}{c|c|c|c|c}
$\begin{array}{c}\text { Very dissa- } \\
\text { tisfied }\end{array}$ & $\begin{array}{c}\text { Dissatis- } \\
\text { fied }\end{array}$ & $\begin{array}{c}\text { Neither satisfied nor } \\
\text { dissatisfied }\end{array}$ & Satisfied & Very satisfied \\
1 & 2 & 3 & 4 & 5
\end{tabular}

F6.4 How satisfied are you with your abilities?

\begin{tabular}{c|c|c|c|c}
$\begin{array}{c}\text { Very dissa- } \\
\text { tisfied }\end{array}$ & $\begin{array}{c}\text { Dissatis- } \\
\text { fied }\end{array}$ & $\begin{array}{c}\text { Nether satisfied nor } \\
\text { dissatisfied }\end{array}$ & Satisfied & Very satisfied \\
1 & 2 & 3 & 4 & 5
\end{tabular}

F7.4 How satisfied are you with the way your body looks?

\begin{tabular}{|c|c|c|c|c|}
\hline $\begin{array}{l}\text { Very dissa- } \\
\text { tisfied } \\
1\end{array}$ & $\begin{array}{l}\text { Dissaris- } \\
\text { fied } \\
2\end{array}$ & $\begin{array}{c}\text { Neither satisfied nor } \\
\text { dissatisfied } \\
3\end{array}$ & $\begin{array}{c}\text { Satisfled } \\
4\end{array}$ & $\begin{array}{c}\text { Very satisfied } \\
5\end{array}$ \\
\hline
\end{tabular}

F10.3 How satisfied are you with your ability to perform your daily living activities?

\begin{tabular}{c|c|c|c|c}
$\begin{array}{c}\text { Very dissam } \\
\text { tisfied }\end{array}$ & $\begin{array}{c}\text { Dissatis- } \\
\text { fied }\end{array}$ & $\begin{array}{c}\text { Neither satisfied nor } \\
\text { dissatisfied }\end{array}$ & Satisfied & Very satisfied \\
1 & 2 & 3 & 4 & 5
\end{tabular}

F13.3 How satisfied are you with your personal relationships?

\begin{tabular}{c|c|c|c|c}
$\begin{array}{c}\text { Very dissa- } \\
\text { tisfled }\end{array}$ & $\begin{array}{c}\text { Dissatis- } \\
\text { fied }\end{array}$ & $\begin{array}{c}\text { Neither satisfied nor } \\
\text { dissatisfied }\end{array}$ & Satisfied & Very satisfied \\
1 & 2 & 3 & 4 & 5
\end{tabular}

F15.3 How satisfied are you with your sex life?

\begin{tabular}{c|c|c|c|c}
$\begin{array}{c}\text { Very dissa- } \\
\text { tisfied }\end{array}$ & $\begin{array}{c}\text { Dissatis- } \\
\text { fied }\end{array}$ & $\begin{array}{c}\text { Neither satisfied nor } \\
\text { dissatisfied }\end{array}$ & Satisfied & Very satisfied \\
1 & 2 & 3 & 4 & 5
\end{tabular}

F14.3 How satisfied are you with the support you get from your family?

\begin{tabular}{c|c|c|c|c}
$\begin{array}{c}\text { Very dissa- } \\
\text { tisfied }\end{array}$ & $\begin{array}{c}\text { Dissatis- } \\
\text { fied }\end{array}$ & $\begin{array}{c}\text { Neither satisfied nor } \\
\text { dissatisfied }\end{array}$ & Satisfied & Very satisfied \\
1 & 2 & 3 & 4 & 5
\end{tabular}


F14.4 How satisfied are you with the support you get from your friends?

\begin{tabular}{c|c|c|c|c}
$\begin{array}{c}\text { Very dissa- } \\
\text { tisfied }\end{array}$ & $\begin{array}{c}\text { Dissatis- } \\
\text { fied }\end{array}$ & $\begin{array}{c}\text { Neither satisfied nor } \\
\text { dissatisfied }\end{array}$ & Satisfied & Very satisfied \\
1 & 2 & 3 & 4 & 5
\end{tabular}

F13.4 How satisfied are you with your ability to provide for or support others?

\begin{tabular}{c|c|c|c|c}
$\begin{array}{c}\text { Very dissa- } \\
\text { tisfied }\end{array}$ & $\begin{array}{c}\text { Dissatis- } \\
\text { fied }\end{array}$ & $\begin{array}{c}\text { Neither satisfied nor } \\
\text { dissatisfied }\end{array}$ & Satisfied & Very satisfied \\
1 & 2 & 3 & 4 & 5
\end{tabular}

F16.4 How satisfied are you with your physical safety and security?

\begin{tabular}{c|c||c|c|c}
$\begin{array}{c}\text { Very dissa- } \\
\text { tistied }\end{array}$ & $\begin{array}{c}\text { Dissatis- } \\
\text { fied }\end{array}$ & $\begin{array}{c}\text { Neither satisfied nor } \\
\text { dissatisfied }\end{array}$ & Satisfied & Very satisfied \\
1 & 2 & 3 & 4 & 5
\end{tabular}

F.17.3 How satisfied are you with the conditions of your living place?

\begin{tabular}{c|c|c|c|c}
$\begin{array}{c}\text { Very dissa- } \\
\text { tisfied }\end{array}$ & $\begin{array}{c}\text { Dissatis- } \\
\text { fied }\end{array}$ & $\begin{array}{c}\text { Neither satisfied nor } \\
\text { dissatisfied }\end{array}$ & Satisfied & Very satisfied \\
1 & 2 & 3 & 4 & 5
\end{tabular}

F18.3 How satisfied are you with your financial situation?

\begin{tabular}{c|c|c|c|c}
$\begin{array}{c}\text { Very dissa- } \\
\text { tisfied }\end{array}$ & $\begin{array}{c}\text { Dissatis- } \\
\text { fied }\end{array}$ & $\begin{array}{c}\text { Neither satisfied nor } \\
\text { dissatisfied }\end{array}$ & Satisfied & Very satisfied \\
1 & 2 & 3 & 4 & 5
\end{tabular}

F19.3 How satisfied are you with your access to health services?

\begin{tabular}{c|c|c|c|c}
$\begin{array}{c}\text { Very dissa- } \\
\text { tisfied }\end{array}$ & $\begin{array}{c}\text { Dissatis- } \\
\text { fied }\end{array}$ & $\begin{array}{c}\text { Neither satisfied nor } \\
\text { dissatisfied }\end{array}$ & Satisficd & Very satisfied \\
1 & 2 & 3 & 4 & 5
\end{tabular}

F19.4 How satisfied are you with the social care services?

\begin{tabular}{c|c|c|c||c}
$\begin{array}{c}\text { Very dissa } \\
\text { tisfied }\end{array}$ & $\begin{array}{c}\text { Dissatis- } \\
\text { fied }\end{array}$ & $\begin{array}{c}\text { Neither satisfied nor } \\
\text { dissatisfied }\end{array}$ & Satisfied & Very satisfied \\
1 & 2 & 3 & 4 & 5
\end{tabular}


F20.3 How satisfied are you with your opportunities for acquiring new skills?

\begin{tabular}{c|c|c|c|c}
$\begin{array}{c}\text { Very dissa- } \\
\text { tisfied }\end{array}$ & $\begin{array}{c}\text { Dissatis- } \\
\text { fied }\end{array}$ & $\begin{array}{c}\text { Neither satisfied nor } \\
\text { dissatisfied }\end{array}$ & Satisfied & Very satisfied \\
1 & 2 & 3 & 4 & 5
\end{tabular}

F20.4 How satisfied are you with your opportunities to learn new information?

\begin{tabular}{c|c|c|c|c}
$\begin{array}{c}\text { Very dissa- } \\
\text { tisfied }\end{array}$ & $\begin{array}{c}\text { Dissatis- } \\
\text { fied }\end{array}$ & $\begin{array}{c}\text { Neither satisfied nor } \\
\text { dissatisfied }\end{array}$ & Satisfied & Very satisfied \\
1 & 2 & 3 & 4 & 5
\end{tabular}

F21.4 How satisfied are you with the way you spend your spare time?

\begin{tabular}{c|c|c|c||c}
$\begin{array}{c}\text { Very dissa- } \\
\text { tisfied }\end{array}$ & $\begin{array}{c}\text { Dissatis- } \\
\text { fied }\end{array}$ & $\begin{array}{c}\text { Neither satisfied nor } \\
\text { dissatisfied }\end{array}$ & Satisfied & Very satisfied \\
1 & 2 & 3 & 4 & 5
\end{tabular}

F22.3 How satisfied are you with your physical enviromment (e.g. pollution, climate, noise, attractivenss)?

\begin{tabular}{c|c|c|c|c}
$\begin{array}{c}\text { Very dissa- } \\
\text { tisfied }\end{array}$ & $\begin{array}{c}\text { Dissatis- } \\
\text { fied }\end{array}$ & $\begin{array}{c}\text { Neither satisfied nor } \\
\text { dissatisfied }\end{array}$ & Satisfied & Very satisfied \\
1 & 2 & 3 & 4 & 5
\end{tabular}

F22.4 How satisfied are you with the climate of the place where you live?

\begin{tabular}{c|c|c|c|c}
$\begin{array}{c}\text { Very dissa- } \\
\text { tisfied }\end{array}$ & $\begin{array}{c}\text { Dissatis- } \\
\text { fied }\end{array}$ & $\begin{array}{c}\text { Neither satisfied nor } \\
\text { dissatisfied }\end{array}$ & Satisfied & Very satisfied \\
1 & 2 & 3 & 4 & 5
\end{tabular}

F23.3 How satisfied are you with your transport?

\begin{tabular}{c|c|c|c|c}
$\begin{array}{c}\text { Very dissa- } \\
\text { tisfied }\end{array}$ & $\begin{array}{c}\text { Dissatis- } \\
\text { fied }\end{array}$ & $\begin{array}{c}\text { Neither satisfied not } \\
\text { dissatisfied }\end{array}$ & Safisfied & Very satisfied \\
1 & 2 & 3 & 4 & 5
\end{tabular}

F13.2 Do you feel happy about your relationship with your familly members?

\begin{tabular}{c|c|c|c|c}
$\begin{array}{c}\text { Very un- } \\
\text { happy } \\
1\end{array}$ & Unhappy & $\begin{array}{c}\text { Neither happy nor } \\
\text { unhappy }\end{array}$ & Happy & Very happy \\
3 & 2 & 3 & 4 & 5
\end{tabular}


Gl How would you rate your quality of life?

\begin{tabular}{|c|c|c|c|c|}
\hline Very poor & Poor & Neither poor nor & Good & Very good \\
\hline 1 & 2 & 3 & 4 & 5 \\
\hline
\end{tabular}

F15.1 How would you rate your sex life?

\begin{tabular}{c|c|c|c|c} 
Very poor & Poor & $\begin{array}{c}\text { Neither poor nor } \\
\text { good }\end{array}$ & Good & Very good \\
1 & 2 & 3 & 4 & 5
\end{tabular}

F3.1 How well do you sleep?

\begin{tabular}{c||c|c|c|c} 
Very poor & Poor & $\begin{array}{c}\text { Neither poor nor } \\
\text { good }\end{array}$ & Good & Very good \\
1 & 2 & 3 & 4 & 5
\end{tabular}

F5.1 How would you rate your memory?

\begin{tabular}{c|c|c|c|c} 
Very poor & Poor & Neither poor nor & Good & Very good \\
1 & 2 & 3 & 4 & 5
\end{tabular}

F19.2 How would you rate the quality of social services available to you?

\begin{tabular}{|c|c|c|c|c|}
\hline Very poor & Poor & Neither poor nor & Grood & Very good \\
\hline $\mathbb{1}$ & 2 & 3 & 4 & 5 \\
\hline
\end{tabular}

The following questions refer to how often you have felt or experienced certain things, for example the support of your family or friends or negative experiences such as feeling unsafe. If you have not experienced these things at all in the last two weeks, circle the number next to the response "never". If you have experienced these things, decide how often and circle the appropriate number. So for exampel if you have experienced pain all the time in the last two weeks circle the number next to "Always". Questions refer to the last two weeks.

F1.1 How often do you suffer (physical) pain?

\begin{tabular}{c|c|c|c|c} 
Never & Seldom & Quite often & Very often & Always \\
1 & 2 & 3 & 4 & 5
\end{tabular}


F4.2 Do you generally feel content?

\begin{tabular}{c|c|c|c|c} 
Never & Seldom & Quite often & Very often & Always \\
1 & 2 & 3 & 4 & 5
\end{tabular}

F8. 1 How often do you have negative feelings, such as blue mood, despair, anxiety, depression?

\begin{tabular}{c|c|c|c|c} 
Never & Seldom & Quite often & Very often & Always \\
1 & 2 & 3 & 4 & 5
\end{tabular}

The following questions refer to any "work" that you do. Work here means any major activity that you do. This includes voluntary work, studying full-time, taking care of the home, taking care of children, paid work or unpaid work. So work, as it is used here, means the activities you feel take up a major part of your time and energy. Questions refer to the last two weeks.

F12.1 Are you able to work?

\begin{tabular}{c|c|c|c|c} 
Not at all & A little & Moderately & Mostly & Completely \\
1 & 2 & 3 & 4 & 5
\end{tabular}

F12.2 Do you feel able to carry out your duties?

\begin{tabular}{c|c|c|c|c} 
Not at all & A little & Moderately & Mostly & Completely \\
1 & 2 & 3 & 4 & 5
\end{tabular}

F12.4 How satisfied are you with your capacity for work?

\begin{tabular}{c|c|c|c|c}
$\begin{array}{c}\text { Very dissa- } \\
\text { tisfied }\end{array}$ & $\begin{array}{c}\text { Dissatis- } \\
\text { fied }\end{array}$ & $\begin{array}{c}\text { Neither satisfied nor } \\
\text { dissatisfied }\end{array}$ & Satisfied & Very satisfied \\
1 & 2 & 3 & 4 & 5
\end{tabular}

F12.3 How would you rate your ability to work?

\begin{tabular}{c|c|c|c|c} 
Very poor & Poor & $\begin{array}{c}\text { Neither poor nor } \\
\text { good }\end{array}$ & Good & Very good \\
1 & 2 & 3 & 4 & 5
\end{tabular}

The next few questions ask about how well you were able to move around, in the last two weeks. This refers to your physical ability to move your body in such a way as to allow you to move about and do the things you would like to do, as well as the things that you need to do. 
F9.1 How well are you able to get around?

\begin{tabular}{c|c|c|c|c} 
Very poor & Poor & Neither poor nor & Good & Very good \\
1 & 2 & 3 & 4 & 5
\end{tabular}

F9.3 How much do any difficulties in mobility bother you?

\begin{tabular}{c|c|c|c|c} 
Not at all & A litule & A moderate amount & Very much & An extreme amount \\
1 & 2 & 3 & 4 & 5
\end{tabular}

F9.4 To what extent do any difficulties in movement affect your way of life?

\begin{tabular}{c|c|c|c|c} 
Not at all & A little & A moderate amount & Very much & An extreme amount \\
1 & 2 & 3 & 4 & 5
\end{tabular}

F9.2 How satisfied are you with your ability to move around?

\begin{tabular}{c|c|c|c|c}
$\begin{array}{c}\text { Very dissa- } \\
\text { tisfied }\end{array}$ & $\begin{array}{c}\text { Dissatis- } \\
\text { fied }\end{array}$ & $\begin{array}{c}\text { Neither satisfied nor } \\
\text { dissatisfied }\end{array}$ & Satisfied & Very satisfied \\
2 & 2 & 3 & 4 & 5
\end{tabular}

The following few questions are concerned with your personal beliefs, and how these affect your quality of life. These questions refer to religion, spirituality and any other beliefs you may hold. Once again these questions refer to the last two weeks.

F24.1 Do your personal beliefs give meaning to your life?

\begin{tabular}{c|c|c|c|c} 
Not at all & A little & A moderate amount & Very much & An extreme amount \\
Il & 2 & 3 & 4 & 5
\end{tabular}

F24.2 To what extent do you feel your life to be meaningful?
Not at all
A little
A moderate amount
Very much
An extreme amount 3

F24.3 To what extent do your personal beliefs give you the strength to face difficulties?

\begin{tabular}{c|c|c|c|c} 
Not at all & A little & A moderate amount & Very much & An extreme amount \\
1 & 2 & 3 & 4 & 5
\end{tabular}

F.24.4 To what extent do your personal beliefs help you to understand difficulties in life?

\begin{tabular}{c|c|c|c|c} 
Not at all & A little & A moderate amount & Very much & An extreme amount \\
1 & 2 & 3 & 4 & 5
\end{tabular}




\section{ABOUT YOU}

What is your gender?

Male

Female

What is your date of birth?

DAY

What is the highest education you received?

Primary school

Secondary school

University

Post-graduate

What is your maritall status?

Single

Married

Living as married

Separated

Divorced

Widowed

How is your health?

\begin{tabular}{c|c|c|c|c} 
Very poor & Poor & Neither poor nor good & Good & Very good \\
1 & 2 & 3 & 4 & 5
\end{tabular}

What health problems do you have at the moment? (TICK NEXT TO THOSE THAT APPLY TO YOU)

. Heart trouble

High blood pressure

Arthritis or Rheumatism

. Cancer

. Emphysema or chronic bronchitis

- Diabetes

- A cataract

Stroke

Broken or fractured bone

Chronic nervous or emotional problems

Chronic foot trouble (bunions, ingrowing toenails)

Rectal growth or rectal bleeding

Parkinson's disease 
. Other (please describe)

Are you currently ill?

. If yes, what is your diagnosis?

Do you have any comments about the questionnaire?

THANK YOU FOR YOUR HELP

WHOQOL group (1995b). Field Trial WHOGOL-100 February 1.995: Facet definitions and questions. Geneva: WHO (MNH/PSF/95.1.B) 


\section{NAWOORD}

Het beschreven onderzoek in dit proefschrift werd geïnitieerd door mijn copromotor, Dr. M. Drent van de vakgroep Longziekten, Rijksuniversiteit Limburg, Maastricht. Marjolein, jou wil ik als eerste hartelijk bedanken. Je plezier in klinisch onderzoek, je onuitputtelijke inzet en enthousiasme hebben mij de afgelopen drie jaar als leidraad gediend. Een betere begeleiding bij het verrichten van onderzoek is niet voorstelbaar. Voor je motiverende, intensieve en persoonlijke inzet - ondanks je eigen werkdrukte - ben ik je veel dank verschuldigd. Door de samenwerking met mijn co-promotor Dr.J. de Vries, van de vakgroep Psychologie van de Katholieke Universiteit Brabant, Tilburg, werd aan het onderzoek een nieuwe dimensie toe gevoegd. Jolanda, bedankt voor je onvoorwaardelijke begeleiding en steun - zelfs in het weekend en gedurende je vakantie. Ik heb genoten van je uitstraling van rust en optimisme.

Prof. dr. E.F.M. Wouters, geachte professor en promotor, u wil ik in het bijzonder bedanken voor de feed-back, de verrassende wendingen en de "finetuning" die u bij de bundeling van de publicaties aanbracht.

Dr. M.H.M. Breteler, Rien, mijn bijzondere dank voor je waardevolle bijdrage aan het opzetten van dit onderzoek en je geduldige begeleiding bij de eerste stappen van het schrijven van een artikel. Met jou kon ik langdurig discussiëren om aan de juiste formulering te komen. Drs. L.M.L. Kock, Leonie, jij bent mij eveneens in het begin zeer behuipzaam geweest met zowel het verzamelen van de data alsmede je statistische vaardigheden-hiervoor mijn bijzondere dank. Dr. P.N.R. Dekhuijzen, Richard, jou wil ik bedanken voor het uitermate snelle en efficiente advies en voor het opzetten van het "Nijmeegse gedeelte" van dit project. Drs. S. Drent, Sylvia, ook jou bedank ik hartelijk voor je waardevolle hulp en het verzamelen van de "Nijmeegse" data. Dr. T.L.Th.A. Jansen, Tim, hartelijk bedankt voor de weliswaar korte, maar uitermate prettige samenwerking; met name voor je hulp en advies bij het opzetten van het "reumatische" gedeelte van dit onderzoek ben ik zeer erkenntelijk. 
Verder wil ik van harte bedanken de collegae van de deelnemende longafdelingen, met name Drs. M.A.M.F. van Nierop (Ziekenhuis Rijnstate, Arnhem), Dr. P.N.R. Dekhuijzen (Academisch Ziekenhuis Nijmegen St. Radboud, Universitair Longcentrum Nijmegen, Groesbeek), Dr. E.J.F.M. ten Berge (Streekziekenhuis Midden Twente, Hengelo), Drs. J.T.H. Hellebrand (St. Jans Gasthuis, Weert), Dr. B. van der Bruggen-Bogaarts (St. Maartens Gasthuis, Venlo), Drs. A.J.Neve (Maasland Ziekenhuis, Sittard) en Dr. M.K.T. Tjwa (De Weverziekenhuis, Heerlen), Drs. L.J.M. Sala en Drs. I. Utama (St. Laurentius Ziekenhuis, Roermond). Zij allen hebben door het verzamelen en ter beschikking stellen van de medische data van "hun" sarcoïdosepatiënten een belangrijke bijdrage geleverd aan dit onderzoek. Natuurlijk was dit onderzoek ook niet mogelijk geweest zonder de medewerking van een groot aantal sarcoïdosepatiënten, die ondanks hun klachten de moeite hebben genomen om tijd en energie ter beschikking te stellen. Deze patiënten alsmede de Besnier Boeck/Sarcoïdose Belangenvereniging Nederland (BBSBN) bedank ik hartelijk voor hun medewerking en steun. De Prof. dr. Jaap Swierenga Stichting gaf een financiele bijdrage waarvoor ik zeer erkentelijk ben. De enquete werd ook mede mogelijk gemaakt door financiële steun van het Ministerie voor Volksgezondheid, Welzijn en Sport.

Valerie Jones, thank you so much for your valuable help and professional editorial advice with respect to the chapters 1 and 3 .

De medewerksters van het secretariaat van de afdeling longziekten van het Academisch Ziekenhuis Maastricht, met name Nicole Genders, bedank ik hartelijk voor de praktische hulp en voor de aangedragen oplossingen van problemen tijdens mijn "werkdagen" in Maastricht. Mevrouw L. Hindley bedank ik hartelijk voor haar advies met betrekking tot de "summary".

Last but not least, mijn paranymfen, Marjon Elfferich en Rob Lamers, jullie bedank ik van harte voor de rust - en steungevende aanwezigheid "achter mijn rug" en voor jullie inzet bij de voorbereidingen van alle activiteiten rond om de promotie. 


\section{CURRICULUM VITAE}

Ruth M. Wirnsberger

17. 2. 1957

1967- 1974

1975- 1976

1976-1984

1985

1986-1987

$1987-1993$

1993-1995

1995

1995-1996

1996

vanaf 1996

1996-1998

1998
Geboren te Villach/Oostenrijk

Middelbare school in Berlin/Duitsland, Concepción/Chili

Studie Tandheelkunde, Universidad de Concepción

Studie Geneeskunde, Universität Wien

Verpleegkundigenwerk, IC hartchirurgie, Allgemeines

Krankenhaus, Wien

Assistente in opleiding tot huisarts, Pulmologisches Zentrum der Stadt Wien, Kaiserin-Elisabeth-Spital

Assistente in opleiding tot longarts, Pulmologisches Zentrum der Stadt Wien

Longarts Sonderkrankenanstalt der Versicherungsgesellschaft der Gewerblichen Wirtschaft, Wien

Registratie longarts in Nederland

Stage afdeling longziekten, OLVG, Amsterdam

Waarneming afdeling longziekten (parttime), ziekenhuis 'de Heel', Zaandam

Promotieonderzoek, vakgroep Pulmonologie, Universiteit Maastricht

Longarts, chef de Clinique, CWZ, Nijmegen

Waarnemend longarts Astmacentrum Heideheuvel 